\title{
DETERMINATION OF THE DESIGN \\ PARAMETERS FOR OPTIMUM \\ HFAVILY LOADED DUCTED FANS
}

\author{
A THESIS \\ Presented to \\ The Faculty of the Graduate Division \\ by \\ Terry Wright \\ In Partial Fulfillment \\ of the Requirements for the Degree \\ Doctor of Philosophy in the School of Aerospace Engineering
}

Georgia Institute of 'Technology

November, 1969 


\section{DETERMINATION OF THE DESIGN \\ PARAMETERS FOR OPTIMUM \\ HEAVILY LOADED DUCTED FANS}

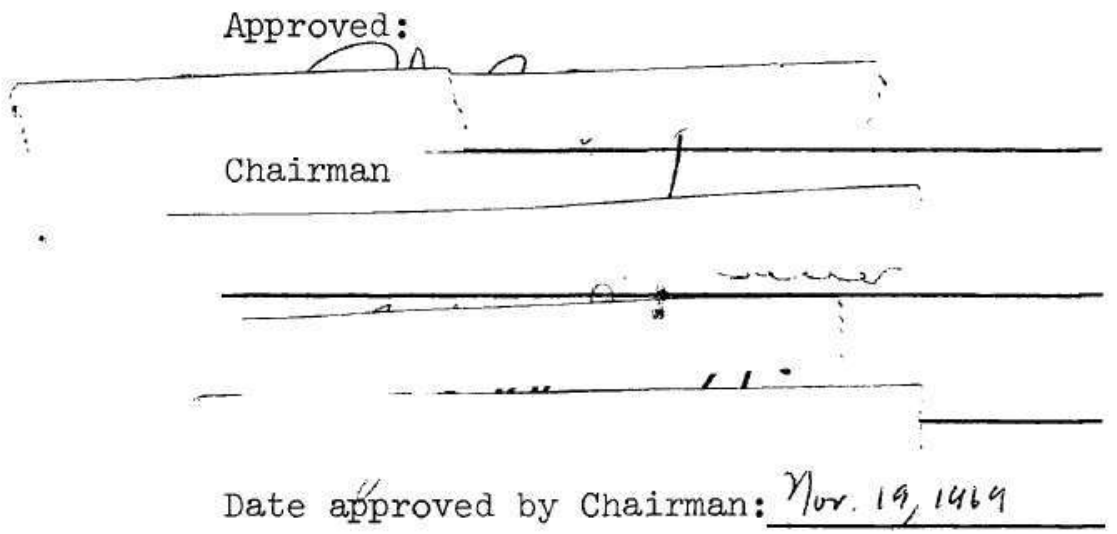


In presenting the dissertation as a partial fulfillment of the requirements for an advanced degree from the Georgia Institute of Technology, I agree that the Library of the Institute shall make it available for inspection and circulation in accordance with its regulations governing materials of this type. I agree that permission to copy from, or to publish from, this dissertation may be granted by the professor under whose direction it was written, or, in his absence, by the Dean of the Graduate Division when such copying or publication is solely for scholarly purposes and does not involve potential financial gain. It is understood that any copying from, or publication of, this dissertation which involves potential financial gain will not be allowed without written permission.

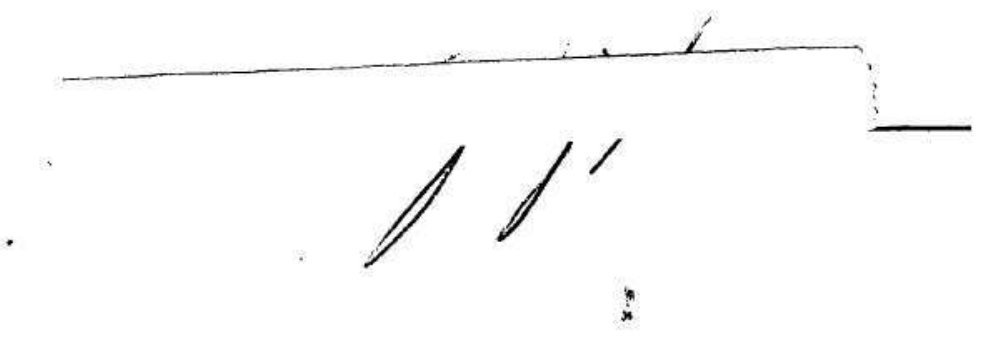

$7 / 25 / 68$ 


\section{ACKINOWLEDGMENTS}

I would like to express my appreciation to Dr. Robin B. Gray for his suggestion of the thesis topic and for his guidarce during the course of this research. I would also like to thank Dr. A. Alvin Pierce and Professor James E. Hubbartt for their caretul examination of the manuscript.

The numerous discussions with Dr. Robert K. Sigman, Mr. C. E. Hammond, Mr. Larry Wright, and my wife Mary Arne have provided me with valuable insight into the various aspects of the problem from time to time. Their advice is greatly appreciated. I thank Dr. Kenton D. Whitehead and Mr. Howard L. Durham for suggestions concerning the computational procedures of the thesis.

The financial support of the National Aeronautics and Space Administration, the U. S. Army Research Office, Durham, and the Geurgia Institute of' Technology are gratefully acknowledged.

Finally, I wish to thank rny parents, Mr. and Mrs. Roy J. Wright, for their assistance and wholehearted support during the earlier years of my education. 
TABLE OF CONTENTS

Page

ACKNOWLEDGMENTS . . . . . . . . . . . . . . . . . . . . . . .
LIST OF TABLES. . . . . . . . . . . . . . . . . . . . . .
LIST OF ILLUSTRATIONS . . . . . . . . . . . . . . . . . . . v vi

NOMENCLATURE. . . . . . . . . . . . . . . . . . . . . . . vili

SUMMARY . . . . . . . . . . . . . . . . . . . . . . . . . Xili

Chapter

I. INTRODUCTION. . . . . . . . . . . . . . . . . . . . .

II. DEVELOPNENT OF THE WAKE VORTEX MODEL. . . . . . . . . . 4

III. ANALYS IS AND SOLUTION OF THE WAKE MODEL . . . . . . . . 24

IV. CALCULATION OF THE THRUST, POWH,

AND INDUCED EFFICIENCY. . . . . . . . . . . . . . 37

Thrust

Power

Efficiericy

V. NUMERICAL PROCEDURES. . . . . . . . . . . . . . 54

Evaluation of the Velocity Components

System of Linear Equations

Thrust and Power Integrations

VI. KESULTS . . . . . . . . . . . . . . . . . . . 74 Appendix

I. GEOMETRY AND MOTION OF THE INNER HELICAL SHEETS . . . . 86

II. VELOCITY FIEID OF THE UNIFORM BOUNDARY SHEET. . . . . .

III. CHECK CASES FOR THE NUMERICAL PROCEDURES. . . . . . . . 98

IV. PERFORMANCE AND DESIGN TABLES. . . . . . . . . . 105 
TABLE OF CONTENTS (Continued)

Page

CITED LITERATURE. . . . . . . . . . . . . . . . . . . . 139

VITA. . . . . . . . . . . . . . . . . . . 141 


\section{IIST OF TABLES}

Table

Page

1. Accuracy of the Wake Integrations . . . . . . . . . 103 


\section{LIST OF ILLUSTRATIONS}

1. Conceptual Diagram of Vortex Shedding at the Duct

Trailing Edge . . . . . . . . . . . . . . . .

2. Helical Coordinate System . . . . . . . . . . 7

3. Ultimate Wake System Showing Paths

of the Line Integrals.... . . . . . . . . .

4. Velocity Diagram at an Inner Helical Vortex Filament of the Ultimate Wake. . . . . . . . . . . . 10

5. Ultimate Wake System Showing Paths of the Line Integrals . . . . . . . . . . . . . 13

6. Concept of the Ultimate Wake Vortex System. . . . . . . 19

(a) Boundary Sheet of Uniform Strength

(b) Boundary sheet of Varying Strength

(c) Result of Superposition

7. Velocity Diagram with respect to the Rotating Blades of the Outermost Filament of the Inner Vortex Sheet and at the Adjacent Point of the Boundary Sheet . . . . 21

8. Control Volume used in Determining the Thrust . . . . 38

9. A Path of Line Integration in the Ultimate Wake . . . . 44

10. Schematic Diagram of the Arrangement of Vortex Filaments and Control Points in the Ultimate Wake of the Ducted Fan. . . . . . . . . . . . . . . . . .

11. Network Used in Calculating the Velocity Integrals on the $\overline{\mathrm{z}} \overline{\mathrm{Y}}$ - Surface. . . . . . . . . . . . . 71

12. Comparison of Two Methods of Determining the Blade Bound Vorticity for a Lightly Loaded Ducted Fan . . . 75

13. Comparison of Two Methods for Determining the Blade Bound Vorticity for a Lightly Loaded Ducted Fan . . . 


\section{LIST OF ILLUSTRATIONS (Continued)}

Figure

14. Comparison of Two Methods of Calculating the Blade Bound Vorticity for a Lightly Loaded Ducted Fan . . .

15. Comparison of Two Methods of Calculating the Blade Bound Vorticity for a Lightly Loaded Ducted Fan . . .

16. Comparison of the Blade Bound Vorticity for a Lightly Loaded Ducted Fan for Successive Numbers of Blades (Digital Computer Solution) with the Infinite Blade Solution (Exact). . . . . . . . . . . . .

17. Comparison of Mass Coefficients for Increasing Numbers of Blades (Digital Computer Solution) with the Mass Coefficient for the Infinite Blade Case (Exact). . . . 80

18. Blade Bound Vorticity Distributions for a Family of Iightly Loaded Ducted Fans. . . . . . . . . . 82

19. Variation of the Load Scaling Factor. . . . . . . . 83

20. Variation of Thrust Coefficient with Load for the Family of Ducted Fans . . . . . . . . . . . 84

21. Variation of the Power Coefficient with Load for the Family of Ducted Fans... . . . . . . . . . . 85

22. Incremental Thrust Efficiency Diagram . . . . . . . . 88

23. Geometry of the Ring-Element Sheet. . . . . . . . . . 92

24. Geometry of the Straight-Line-Element Sheet . . . . . 95

25. Schematic Arrangement of Filaments and Control Point in the Ultimate Wake of a Free Propeller. . . . . . . . 100

26. Comparison of Two Methods for Calculating the Blade Bound Vorticity for a Free Propeller. . . . . . . . . . 101

27. Convergence of the Wake Integrations to the Inxact Results for the Infinite Blade Case . . . . . . . . . . 104 


\section{NOMENCLATURE}

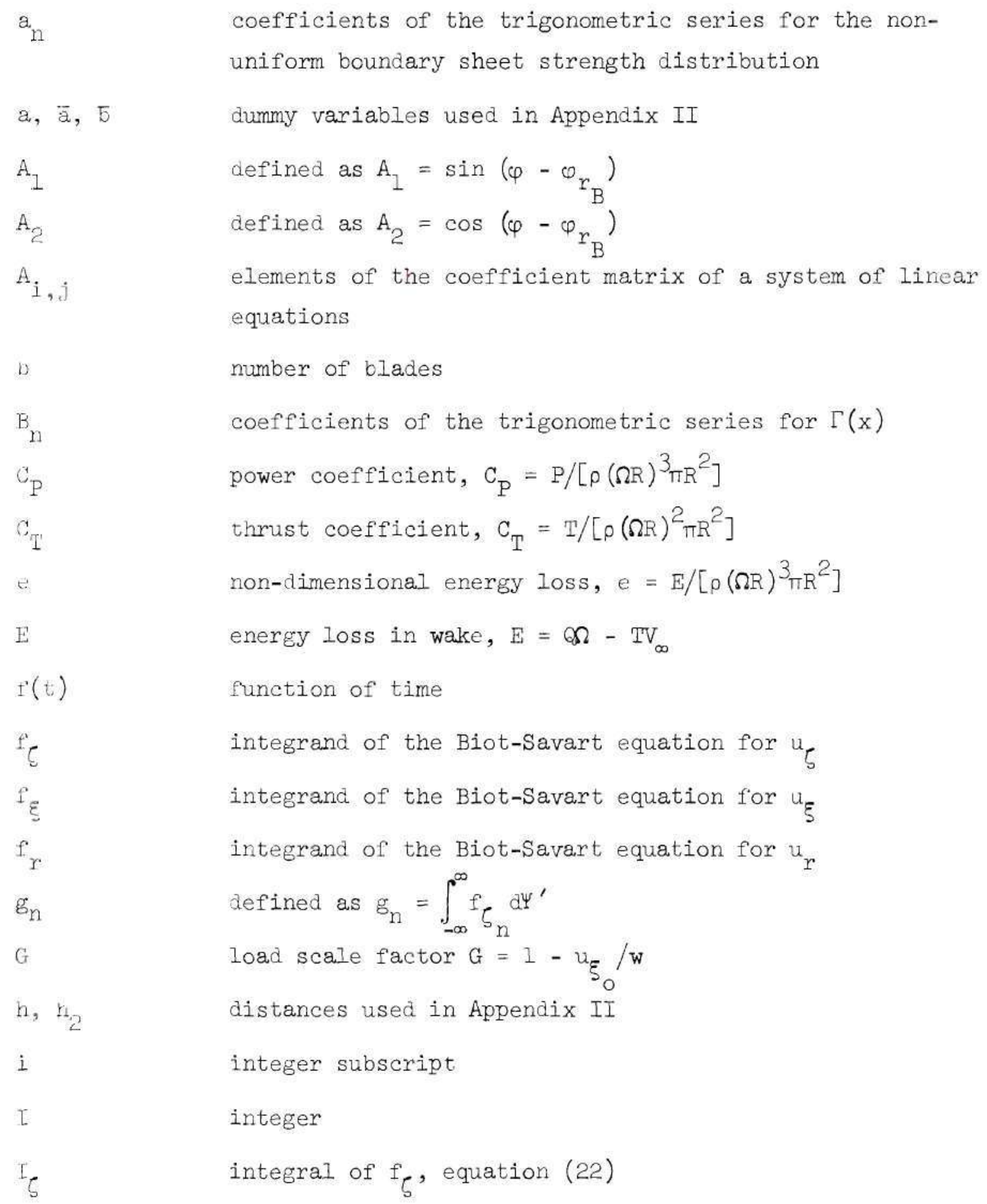

$a_{n}$

coefficients of the trigonometric series for the nonuniform boundary sheet strength distribution dummy variables used in Appendix II

defined as $A_{1}=\sin \left(\varphi-\omega_{r_{B}}\right)$

defined as $A_{2}=\cos \left(\varphi-\varphi_{r_{B}}\right)$

elements of the coefficient matrix of a system of linear equations

number of blades

coefficients of the trigonometric series for $\Gamma(x)$

power coefficient, $C_{P}=P /\left[\rho(\Omega R)^{3} R^{2}\right]$

thrust coefficient, $C_{T}=T /\left[\rho(\Omega R)^{2} \pi R^{2}\right]$

non-dimensional energy loss, $e=E /\left[\rho(\Omega R)^{3} \pi R^{2}\right]$

energy loss in wake, $E=Q \Omega-T V_{\infty}$

function of time

integrand of the Biot-Savart equation for $u_{\zeta}$ integrand of the Biot-Savart equation for $u_{\xi}$ integrand of the Biot-Savart equation for $u_{r}$ defined as $g_{n}=\int_{-\infty}^{\infty} f_{\zeta_{n}} d \Psi^{\prime}$

load scale factor $G=1-u_{\xi_{0}} / w$

distances used in Appendix II

integer subscript

integer

integral of $f_{\zeta}$, equation (22) 


\section{NOMENCLATURE（Continued）}

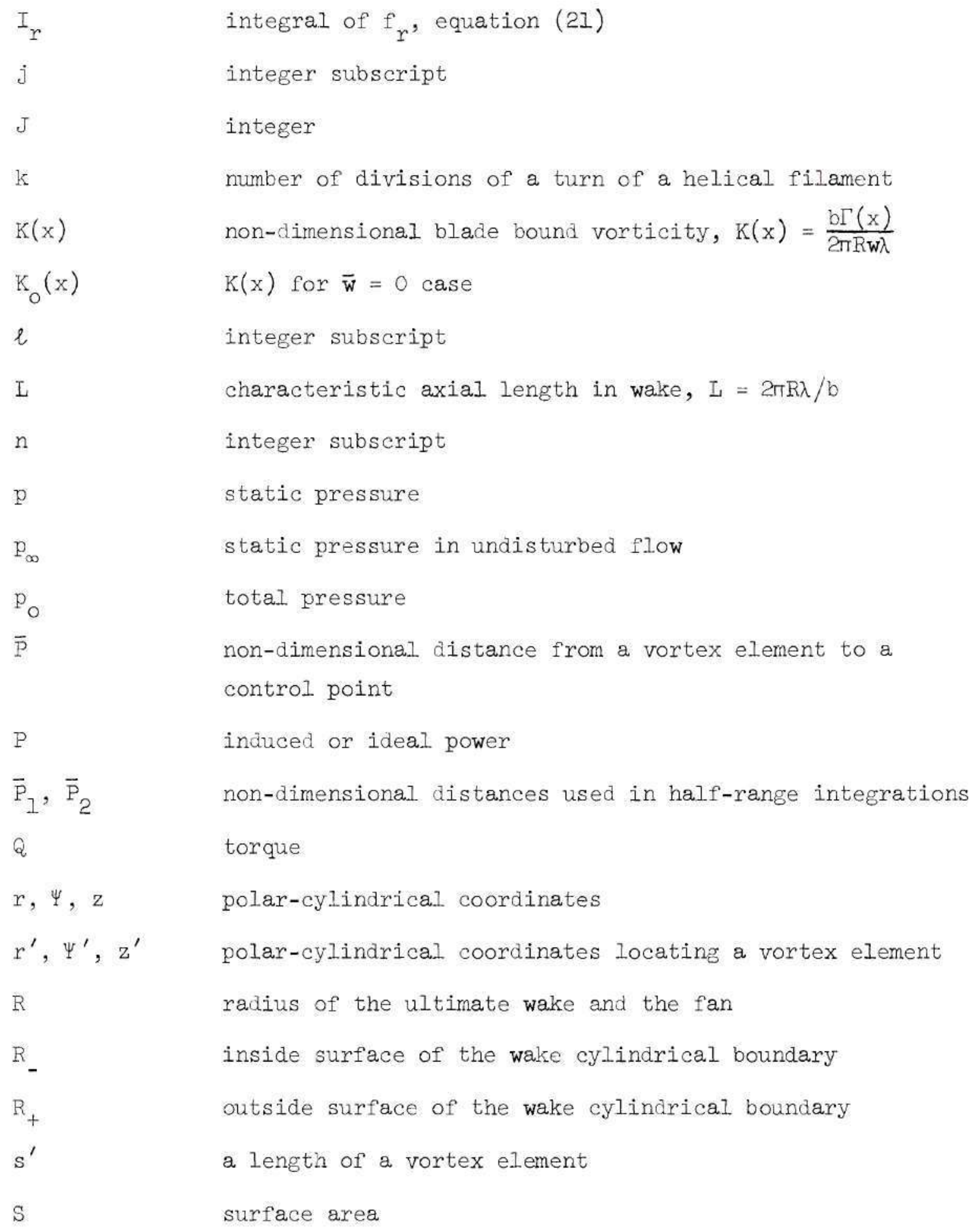




\section{NOMENCLATURE (Continued)}

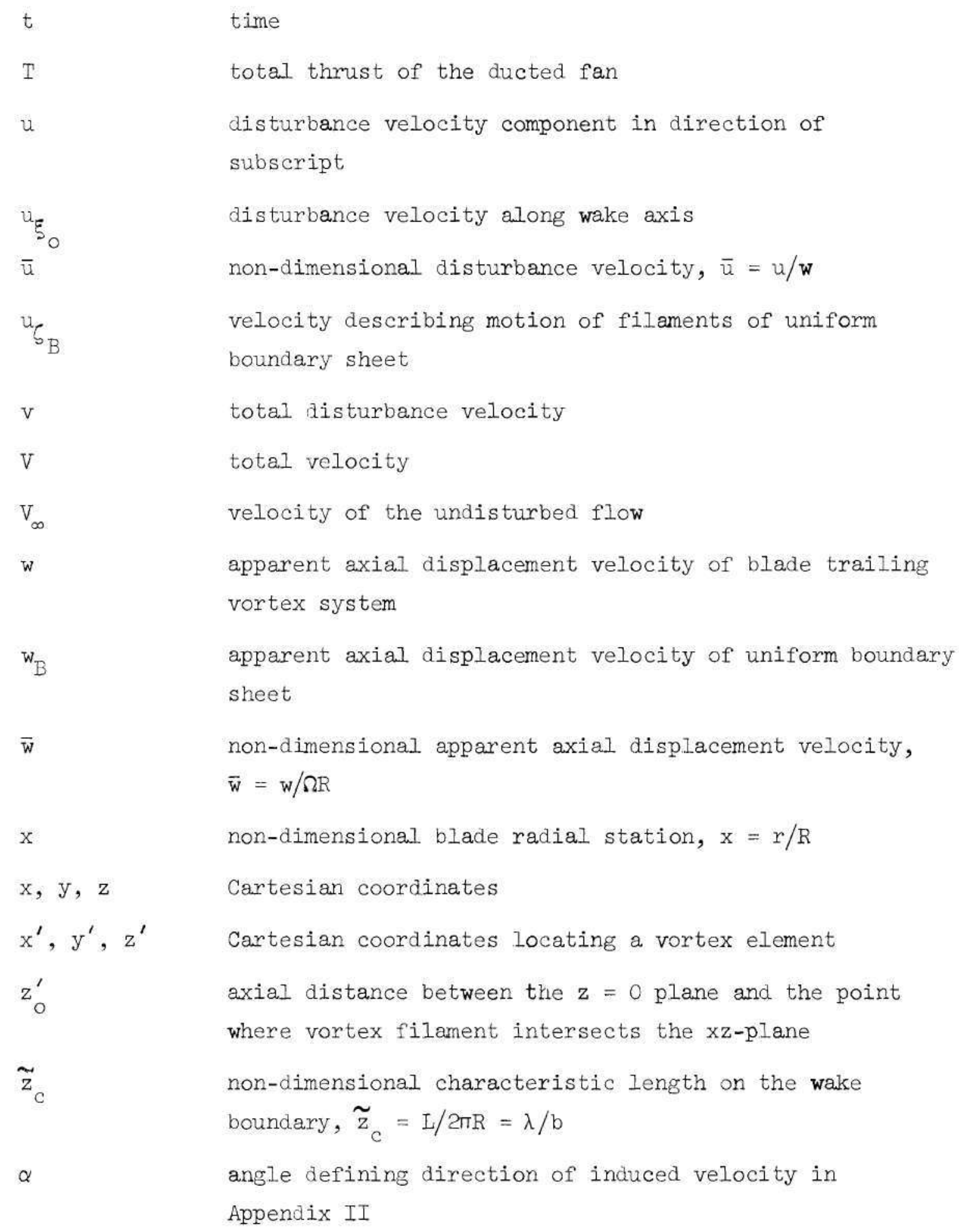


NOMENCLATURE (Continued)

$\beta$

$\gamma$

$\bar{\gamma}$

$\tilde{\gamma}$

$Y\left(\zeta_{B}\right)$

$\Gamma(\mathrm{x})$

$\delta_{\ell}(\Delta u / w)$

$\epsilon$

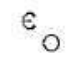

$\epsilon_{r}$

$\epsilon_{\Psi}$

$\varepsilon_{Z}$

$\epsilon_{R}$

5

$n_{i}$

飞

ko

$\lambda$ angle between $d s^{\prime}$ and $\overline{\mathrm{P}}$

a vortex filament strength

non-dimensional vortex filament strength, $\bar{\gamma}=\gamma /(4 \pi R w \lambda G)$

vortex sheet strength per unit length normal to the

filaments

vortex sheet strength of the uniform boundary sheet

blade bound vortex strength distribution

contribution of the $\ell$ th turn of a helical vortex

filament to $\Delta u / w$

defined as $\epsilon=\epsilon_{r}+\epsilon_{\Psi}+\epsilon_{z}$

numerical factor for $\mathrm{C}_{\mathrm{T}}$, equation (45)

defined as $\epsilon_{r}=\int_{0}^{1} \int_{0}^{1} \int_{0}^{2 \pi} \bar{u}_{r}^{2} \bar{r} d \vec{r} d \bar{z} d \Psi / 2 \pi$

defined as $\epsilon_{\Psi}=\int_{0}^{1} \int_{0}^{1} \int_{0}^{2 \pi} \bar{u}_{\Psi}^{2} \bar{r} d \bar{r} d \bar{z} d \Psi / 2 \pi$

defined as $\epsilon_{z}=\int_{0}^{1} \int_{0}^{1} \int_{0}^{2 \pi} \bar{u}_{z}^{2} \bar{r} d \bar{r} d \bar{z} d \Psi / 2 \pi$

defined as $\epsilon_{R_{-}}=\int_{0}^{1} \int_{0}^{1} \int_{0}^{2 \pi}\left[\bar{u}_{z_{R}}^{2}+\bar{u}_{\Psi_{R}}^{2}\right] \bar{r} d \bar{r} d \bar{z} d \Psi / 2 \pi$

helical coordinate normal to the filaments

induced efficiency, $\eta_{i}=(\lambda-\overrightarrow{\mathrm{w}}) \mathrm{C}_{\mathrm{T}} / \mathrm{C}_{\mathrm{P}}$

mass coefficient, $\kappa=2 \int_{0}^{1} K(x) x d x$

$k$ for $\overline{\mathrm{w}}=0$ case

pitch of the inner helical sheets, $\lambda=\left(V_{\infty}+w\right) / \Omega R$ 
NOMENCLATURE (Continued)

\begin{tabular}{|c|c|}
\hline$\lambda_{B}$ & $\begin{array}{l}\text { pitch of the uniform boundary sheet filaments, } \\
\lambda_{B}=\left(V_{\infty}+w_{B}\right) / \Omega R\end{array}$ \\
\hline$\mu$ & defined as $\mu=2 \int_{0}^{1} K(x) \frac{x}{x^{2}+\lambda^{2}} d x$ \\
\hline$\mu_{0}$ & $\mu$ lor $\overrightarrow{\mathrm{w}}=0$ case \\
\hline$\rho$ & fluid density \\
\hline$\varphi$ & pitch angle of an inner helical sheet at $r$ \\
\hline$P_{\mathrm{R}}$ & pitch angle of an inner helical sheet at $r=R$ \\
\hline$\varphi_{B}$ & pitch angle of the uniform boundary sheet \\
\hline$\varphi^{\prime}$ & $\begin{array}{l}\text { pitch angle of a filament of an inner helical sheet } \\
\text { at } r^{\prime}\end{array}$ \\
\hline$\omega_{r_{B}}$ & $\begin{array}{l}\text { dummy pitch angle defined by } \varphi_{r_{B}}=\tan ^{-1}\left(\tan \varphi_{B} / \bar{r}\right) \\
\text { disturbance velocity potential }\end{array}$ \\
\hline$\Psi_{0}$ & an aruitrarily fixed polar angle \\
\hline$\omega$ & half-width of a strip element of a vortex sheet \\
\hline$\Omega$ & blade rotational speed (radians per second) \\
\hline
\end{tabular}




\section{SUMMARY}

Like the free propeller in axial flight, a single-rotation ducted fan of highest induced efficiency is characterized by an ultimate wake vortex system shed from the blade trailing edges whose apparent motion is that of rigid helical surfaces. In addition and concentric with this inner sheet system there is a cylindrical surface of helical vortex filaments shed from the duct trailing edge. For zero hub diameter and neglecting compressibility, viscosity, and tip clearance, a consistent mathematical model of the constant-diameter vortex wake is developed and the compatibility relationships to be satisfied are presented. Using the Biot-Savart equation, the vortex strength distribution in the ultimate wake is determined and then related to the blade bound vortex strength distribution. In addition, expressions are developed for the thrust, power, and induced efficiency which depend on numerical integrations of velocity profiles in the ultimate wake. It is shown that the wake vorticity and the velocity distribution in the wake, for all loadings from the lightly loaded limit to the heavily loaded static thrust condition may be extracted from the lightly loaded result through the use of a simple algebraic scale factor. The performance parameters, thrust, power and blade bound vortex strength, are thus expressed in terms of a lightly loaded solution for a given freestream velocity, blade tip speed, blade number and the loading parameter. The lightly Ioaded case is compared to existing theoretical and experimental 
results. Some samplo results for heavily loaded performance parameters and design parameters are presented and compar'ed to the exact results for a heavily loaded lucted fan with an infinite number of blades. Results for the heavily loaded system over a broad range of conditions are presented. 


\section{CHAPTER I}

\section{INTRODUCTION}

In recent years there has been an increasing interest in low speed thrust and high-lift devices for application to aircraft with short or vertical take-off and landing characteristics. One of the devices under consideration for this role is the ducted fan or shrouded propeller. Analytical and experimental work in this field have been summarized in an excellent review of the state of the art through 1963 by Sacks and Burnell $I^{1}$. As pointed out in the review, there have existed for some time adequate means of sizing and designing a duct for a given choice of fan blade design but there has been no method available for optimizing the blade design for the finite bladed system which operates at any but the lightest loadings. For the lightly loaded case the problem has been solved by Tachmindji ${ }^{2}$, Theodorsen ${ }^{3}$, and Gray ${ }^{4,5}$. In more recent years Morgan ${ }^{6}$, Ordway, Sluyter, and Sonnerup ${ }^{7}$, Chaplin ${ }^{8}$, and others have published papers in the field of ducted fans, still with primary emphasis on the shroud design. It is the purpose of this research to provide the information needed to size and design the optimum heavily loaded ducted fan.

The basis for this work stems from the classical analysis of the free propeller. It has been shown by Betz ${ }^{9}$ that an isolated propeller having the highest possible induced efficiency, that is, an optimum free propeller, will generate an ultimate wake vortex 
system which moves through the fluid medium as if the vortex sheets of the wake formed a rigid helical structure of constant pitch. Application of this constraint to the motion of the sheets provides a straightforward means of determining the radial distribution of shed vortex sheet strength distribution. This determination has been carried out by Goldstein ${ }^{10}$ and Theodorsen ${ }^{11}$.

It has been shown by Gray ${ }^{4}$ that, for the ducted fan having the highest possible induced efficiency, the same arguments and considerations aro valid concerning the geometry and motion of those vortex sheets which are shed from the blade trailing edges. These arguments are reprortuced in Appendix I for completeness.

In addition to this constraint, the system must satisfy the Kutta condition at the duct trailing edge; that is, the flow at the trailing edge must be tangent to the duct mean camber surface. As a consequence of this condition a sheet of vorticity must be shed from the trailing edge forming a boundary sheet enclosing the screw-like sheets shed from the blade trailing edges. It is the determination of the geometry, motion, and strength distribution of this boundary sheet and its mutual interaction with the inner sheets which constitute the development of the ultimate wake vortex model for the ducted fan.

When the ceometry and motion of the vortex sheets of the ultimate wake have been defined, a straightforward application of the Biot-Savart equation determines the strength distribution of the vortex sheets. The blade bound circulation or vortex strength distribution is then obtained by integration of the inner helical sheet strength. This bound circulation is the basic information from which the fan blades 
can be designed using any of the methods available in the literature (e.g. Theodorsen ${ }^{11}$ ). In addition, the vortex sheet strength distributions enable a detailed calculation of the flow in the wake from which the thrust and induced power may be calculated.

In the development and analysis which follows the effects of hub diameter, blade tip clearance, compressibility and viscosity are neglected. The results will therefore give an upper limit on the performance of single-rotation fans without stator vanes for comparison with other design methods. 


\section{CHAPTER II}

\section{DEVELOPMENT OF THE WAKE VORTEX MODEL}

Earlier analyses by Gray (see Appendix I) have shown that the optimum condition is obtained for the ducted fan when the helical vortex sheets shed from the blades appear to move as rigid screw surfaces in the ultimate wake. The principle difference between the arguments used by Gray and those for the free propeller is that the induced velocity in the wake, at the surface of a helical vortex sheet, need not be normal to the sheet surface. Beyond the need for satisfying the Kutta condition at the duct trailing edge no other information is obtained and actitional relationships must be developed in order to assure a compatiblo vortex model.

Further information may be obtained through consideration of the flow associated with the vortex systems. A blade trailing vortex sheet has large radial velocity components associated with it and, with respect to a coordinate system fixed in the duct, this flow is periodic. Consequently, if the duct mean camber surface is to correspond to a strcamline, a distribution of vorticity must be placed along this surface to eancel all normal velocity components. Part of this distribution must he periodic in nature and must also be a function of the number of bladis in the fan and must rotate with the blades. A portion of this periolic distribution is considered to be a continuation of each blade bound vortex which passes directly from the blade tip onto the duct 
contour where it spreads out over the duct mean camber surface. Then, in accordance wi ith the Helmholtz theorems, it moves aft along the mean camber surface ar: is shed at the duct trailing edge as free vortex filaments of varying geometry and density wrapped on a cylindrical surface. While these filaments are within the duct contour it is assumed that th mak all of the necessary adjustments in phase relationship and tensity with the blade trailing vortex system so that the motion of this wake boundary system is along the tangent to the mean camber curface at the trailing edge of the duct as shown in Fig. 1. In addition to the blade bound vortex continuation, a portion of the tuct trailing vortex sheet is considered to be shed from bound vortices of vary ing strength rotating in the duct with the blades. It is also assumen that the duct is designed so that there is no contraction or expansion of the wake downstream of the fan. This is a feasibl design problem ${ }^{8}$ and guarantees that the duct is compatible with the wake gcometry of the analysis.

Continuing with the examination of the velocities associated with the wake vortex sheets, consider a helical coordinate system $r, \bar{\zeta}, \zeta$ defined in terms of the cylindrical coordinates $r, \Psi, z$ as shown in Pig. 2. At a givn instant an inner helical vortex sheet in the ultimate wake coincides with the $\zeta=0$ surface. Then

$$
\begin{gathered}
r=r \quad, \quad 0 \leq r \leq \infty \\
\xi=r \Psi \cos \varphi+z \cos \varphi,-\infty \leq \xi \leq \infty \\
\zeta=-r \Psi \sin \theta+z \cos \varphi, \frac{-\left(V_{\infty}+w\right) \cos \varphi}{2 b(\Omega / 2 \pi)} \leq \zeta \leq \frac{\left(V_{\infty}+w\right) \cos \varphi}{2 b(\Omega / 2 \pi)}
\end{gathered}
$$




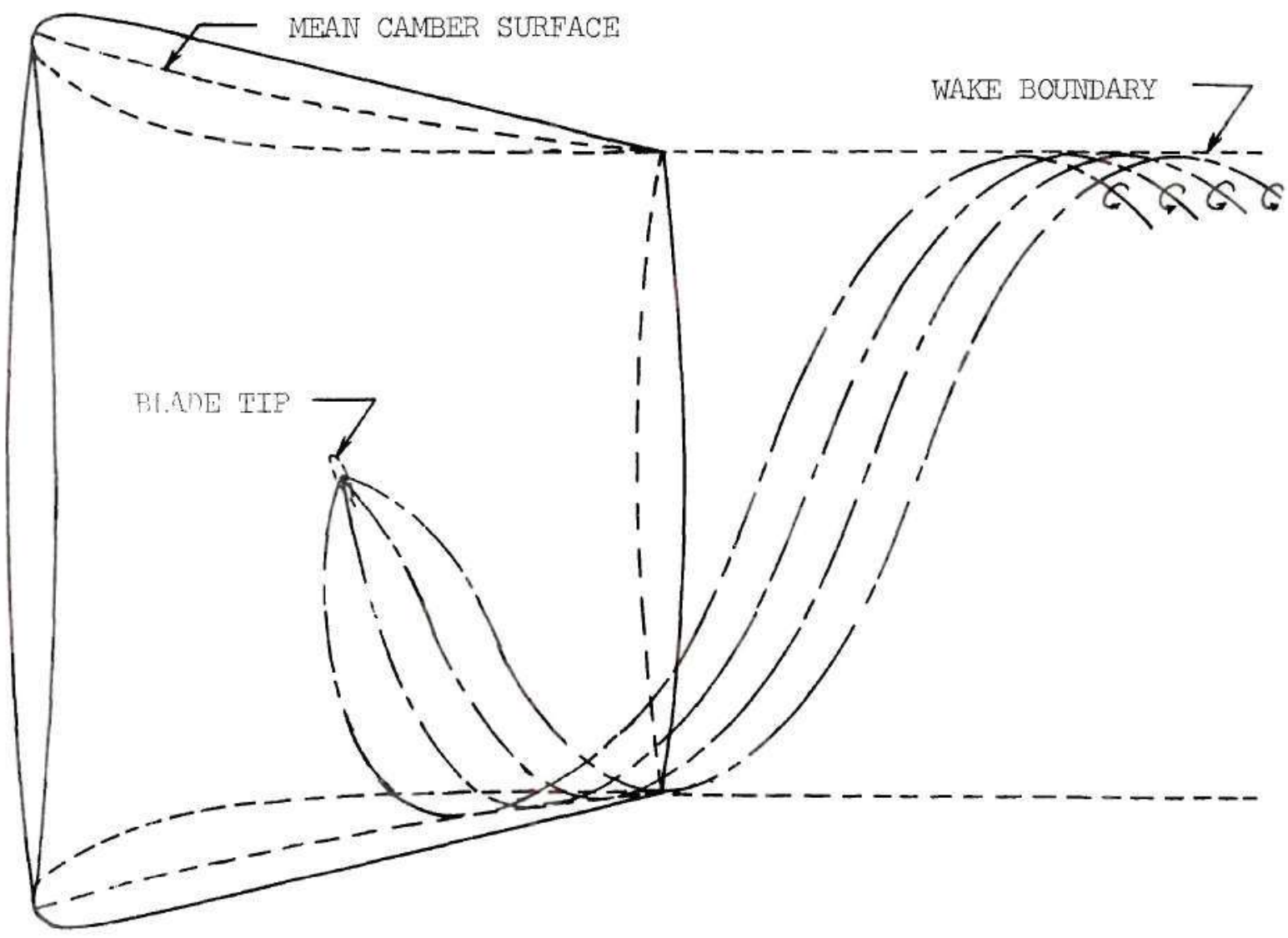

Pigur. L. Conceptual. Diagram of Vortex Sheduing at the Duct Trailing Edge. 


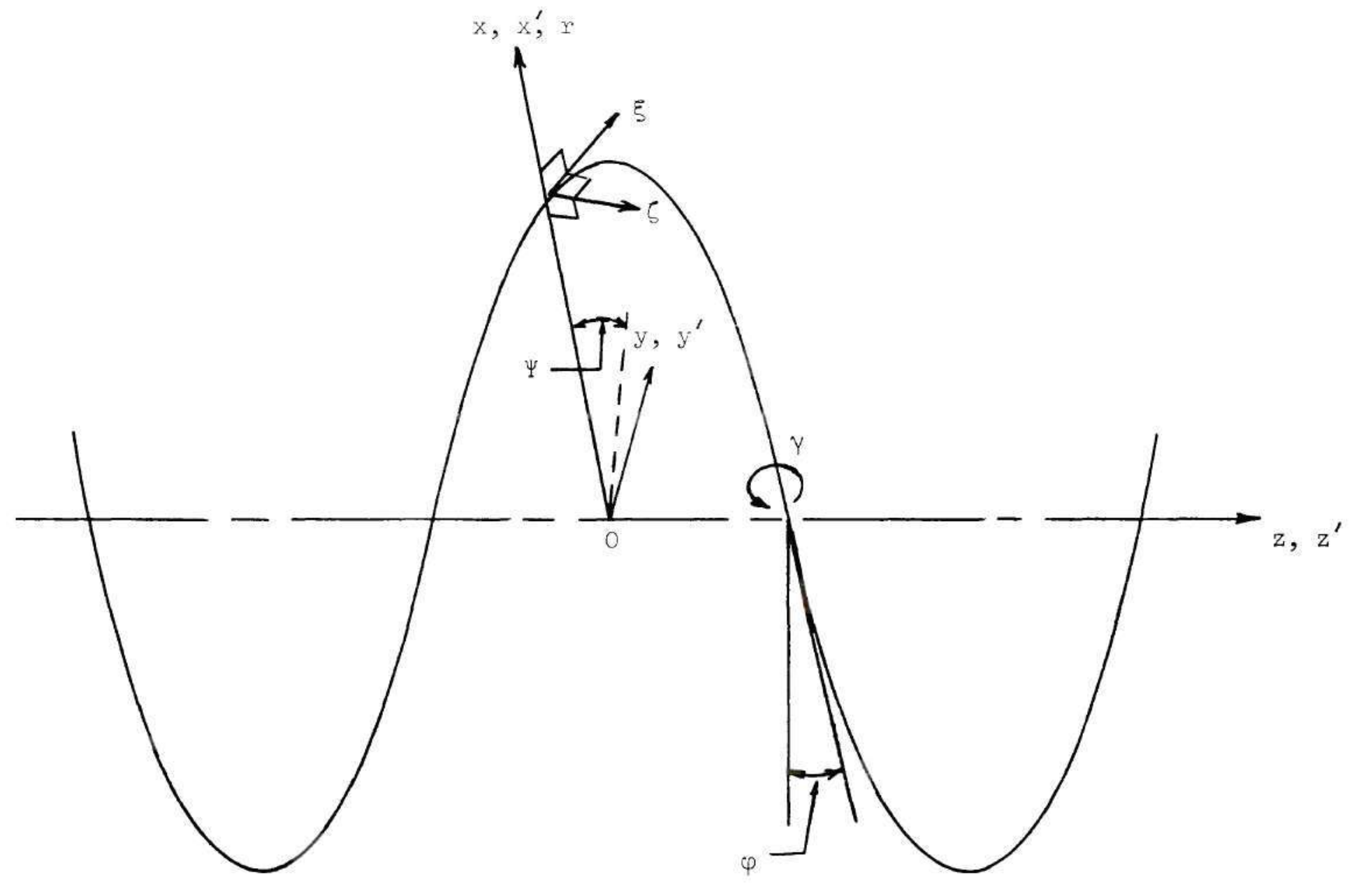

Figure 2. Helical Coordinate System. 
where $\xi$ is measured along the helical vortex filaments and $\zeta$ is measured along a helical line normal to the vortex filaments. It should be noted at the outset that $\mathrm{w}$, the apparent velocity parameter, is not an actual disturbance velocity associated with the vortex sheets of the wake. Rather, it is the speed along the wake axis with which the inner helical sheets appear to be moving relative to a coordinate system fixed in space. Similarly $\left(V_{\infty}+w\right)$ is the axial speed with which the inner sheets appear to move relative to the ducted fan.

If the apparent rigid stmucture of the inner helical sheets in the ultimate wake is to be maintained, an observer fixed to any point on a sheet must see the same distribution of vorticity in the wake regardless of his angular position on the sheet. He must further see the same distribution above as he sees below. These constraints on the distribution of vorticity preclude the possibility of radial distortion of the inner sheets and amount to a requirement of helical symmetry of the wake vortex system. Subject to this helical symmetry the disturbance velocity vector will be constant along the helical lines $r=$ constant and $\zeta=$ constant both inside and outside the wake. Now, consider a line integral of the velocity along the path $\overline{\mathrm{ABCDA}}$ within the ultimate wake as shown in Fig. 3. The velocity diagram with respect to the rotating blades is shown in Fig. 4 . Along $\overline{\mathrm{BC}} \mathrm{u}_{\xi}$ is constant since $r$ and $\zeta$ are constant. $\overline{\mathrm{AB}}$ and $\overline{\mathrm{CD}}$ are radial lines at two values of $\Psi$ on the sheet surface and $\overline{D A}$ coincides with the wake axis. Since no vorticity is enclosed by the path, the line integral. is zero so that 


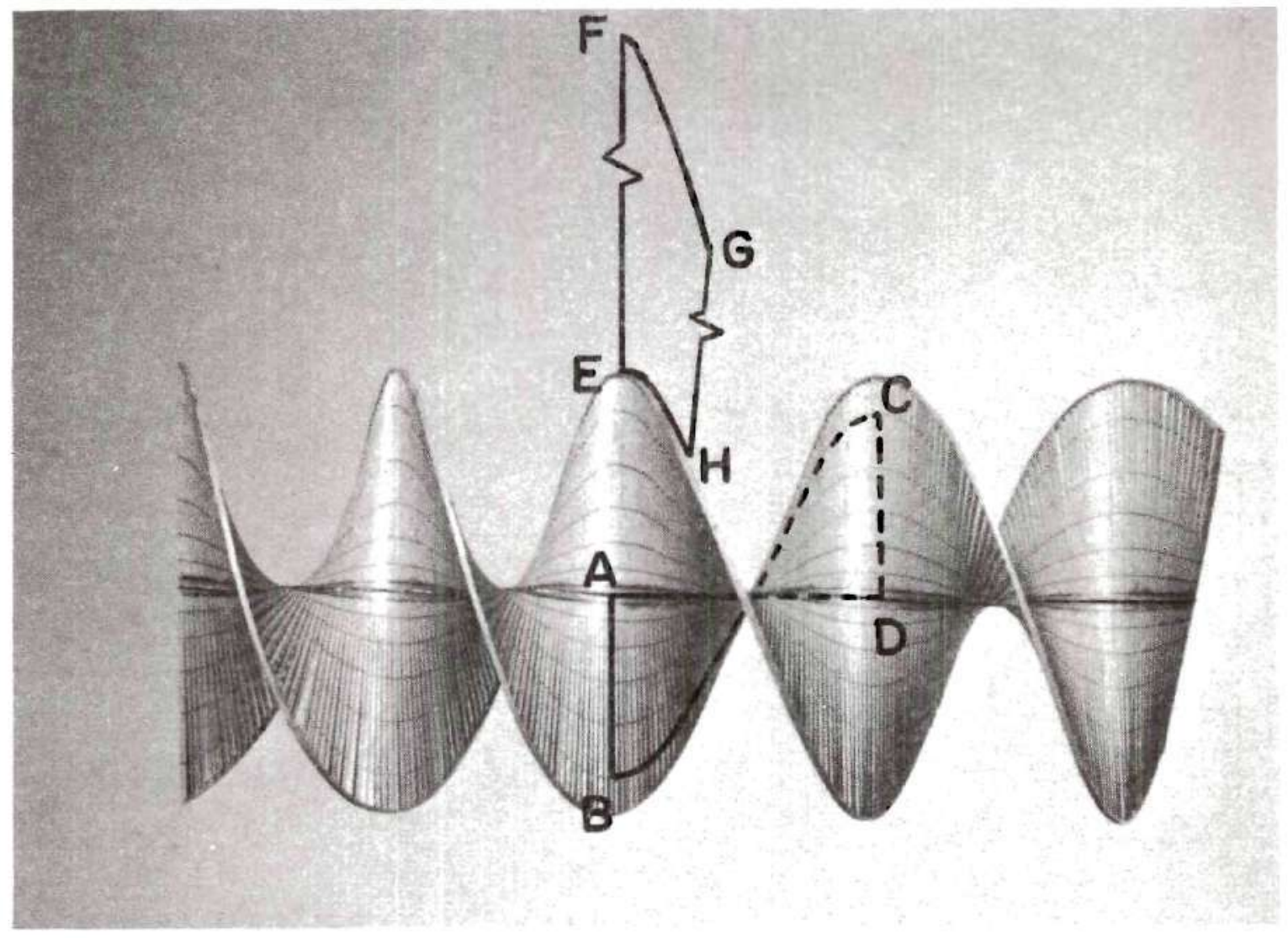

Fig. 3. Ultimate Wake System Showing Paths of Iine Integrals. 


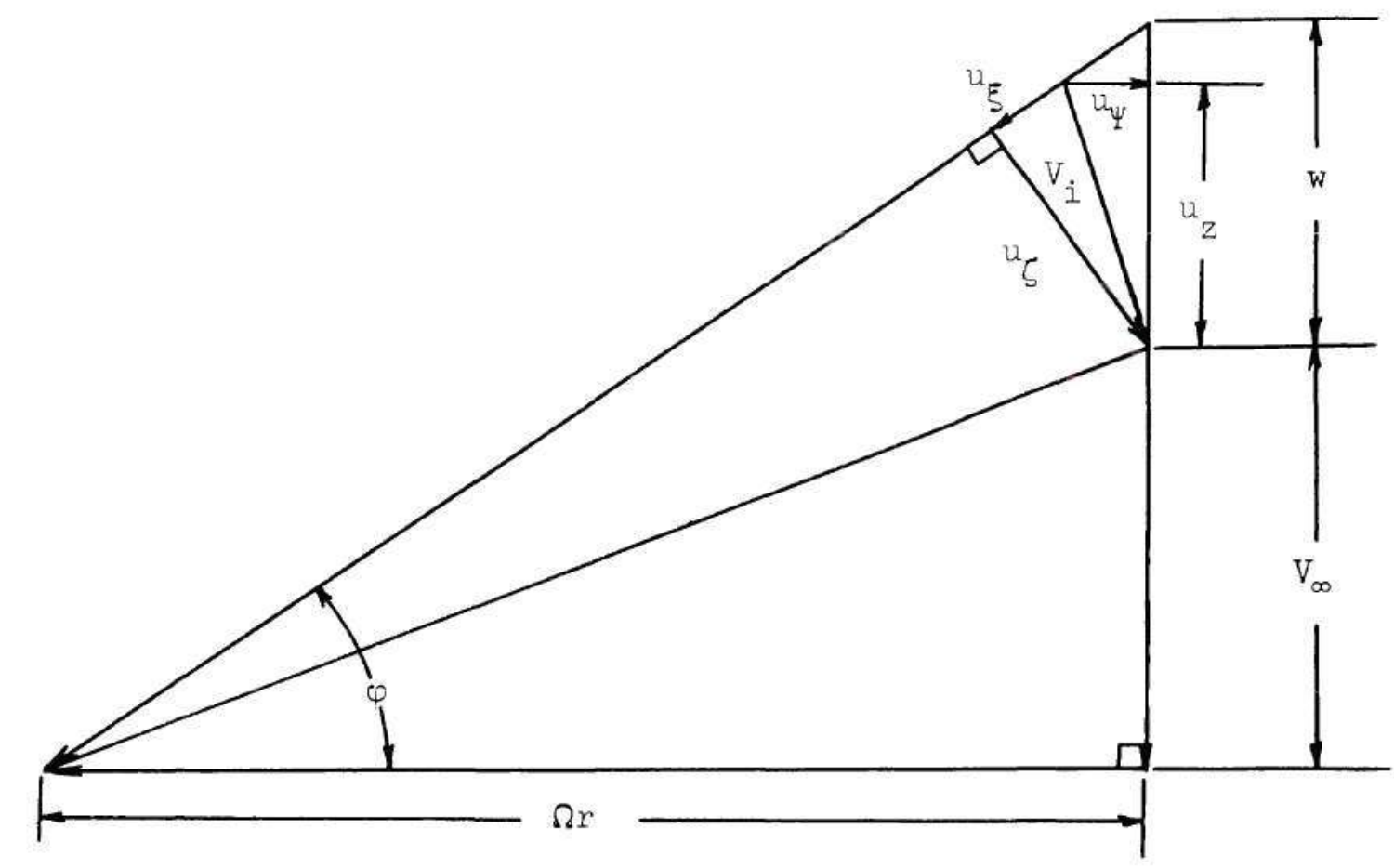

Figure 4. Velocity Diagram at an Inner Helical Vortex Filament of the Ultimate Wake. 


$$
\int_{A}^{B} u_{r} d r+\int_{B}^{C} u_{\xi} d \xi+\int_{C}^{D} u_{r} d r+\int_{D}^{A} u_{\xi} d \xi=0
$$

or

$$
u_{\xi}\left(\xi_{C}-\xi_{B}\right)+u_{\xi}\left(z_{A}-z_{D}\right)=0
$$

where $u_{\xi_{0}}$ is the value of $u_{\xi}$ on the axis $(x=0)$.

Employing the helical coordinate relationships

$$
-u_{\xi}\left(r \frac{2 \pi}{b} \cos \varphi+\frac{V_{\infty}+w}{b(\Omega / 2 \pi)} \sin \rho\right)+u_{\xi} \frac{V_{\infty}+w}{b(\Omega / 2 \pi)}=0 .
$$

Then

$$
u_{\xi}=u_{\xi}\left\{\frac{\left(v_{\infty}+w\right) / \Omega R}{\frac{r}{R} \cos \varphi+\frac{V_{\infty}+w}{\Omega R} \sin \varphi}\right\}
$$

but $\tan \varphi_{\mathrm{R}}=\left(\mathrm{V}_{\infty}+\mathrm{w}\right) / \Omega \mathrm{R}$ and for the coordinate $\zeta$

$r \tan \theta=R \tan \theta_{R}$ so that

$$
u_{\xi}=u_{\xi_{0}}\left\{\frac{\tan \varphi}{\cos \varphi+\tan \varphi \sin \varphi}\right\}
$$

or

$$
u_{\xi}=u_{\xi_{0}} \sin \varphi .
$$

Consider rext the line integral along the path $\overline{\text { EFGHE }}$ as shown in Fig. 3. The portion $\overline{\mathrm{EH}}$ is at $r=R_{-}$. From the requirement of helical symmetry, the filaments of the boundary vortex sheet must cross the line of intersection with an inner helical sheet at every 
point on the intersection line at the same filament pitch angle regardless of angular position. In particular, if the pitch angle of the boundary sheet filaments is identical to that of the inner sheet as the boundary is reached $(r=R)$ then no vorticity is enclosed by the path so that the line integral is zero. (The equal pitch constraint is a lightly loaded condition which will be relaxed later). Thus

$$
u_{\xi}\left(\xi_{G}-\xi_{F}\right)+u_{\xi_{O}} \sin \varphi_{R}\left(\xi_{E}-\xi_{H}\right)=0
$$

or

$$
{ }^{u_{\xi}}=u_{\xi_{0}} \sin \varphi
$$

outside the wake as weIl as inside.

Further, the path $\overline{\mathrm{ABCDA}}$ may be shifted axially so that the helical portion of the path lies along an arbitrary helical line at the pitch angle $\omega$ anywhere inside the wake. The restrictions of symmetry still require $u_{\xi}$ to be constant along this path so that $u_{\xi}=u_{\xi} \sin \varphi$ throughout the wake.

These results are subject to the additional constraint that the line integral of velocity taken along a path enclosing the wake must be zero. The line integral may then be taken along the path $\overline{\mathrm{ABCA}}$ in Fig. 5 where $u_{\xi}$ along $\overline{\mathrm{ABC}}$ is constant and known so that

$$
\int_{C}^{A} u_{z} d z+2 \pi R u_{\xi_{0}} \tan \varphi_{R}=0
$$

The integral is then taken along $\overline{\text { ACDEA}}$. Subject to helical symmetry 


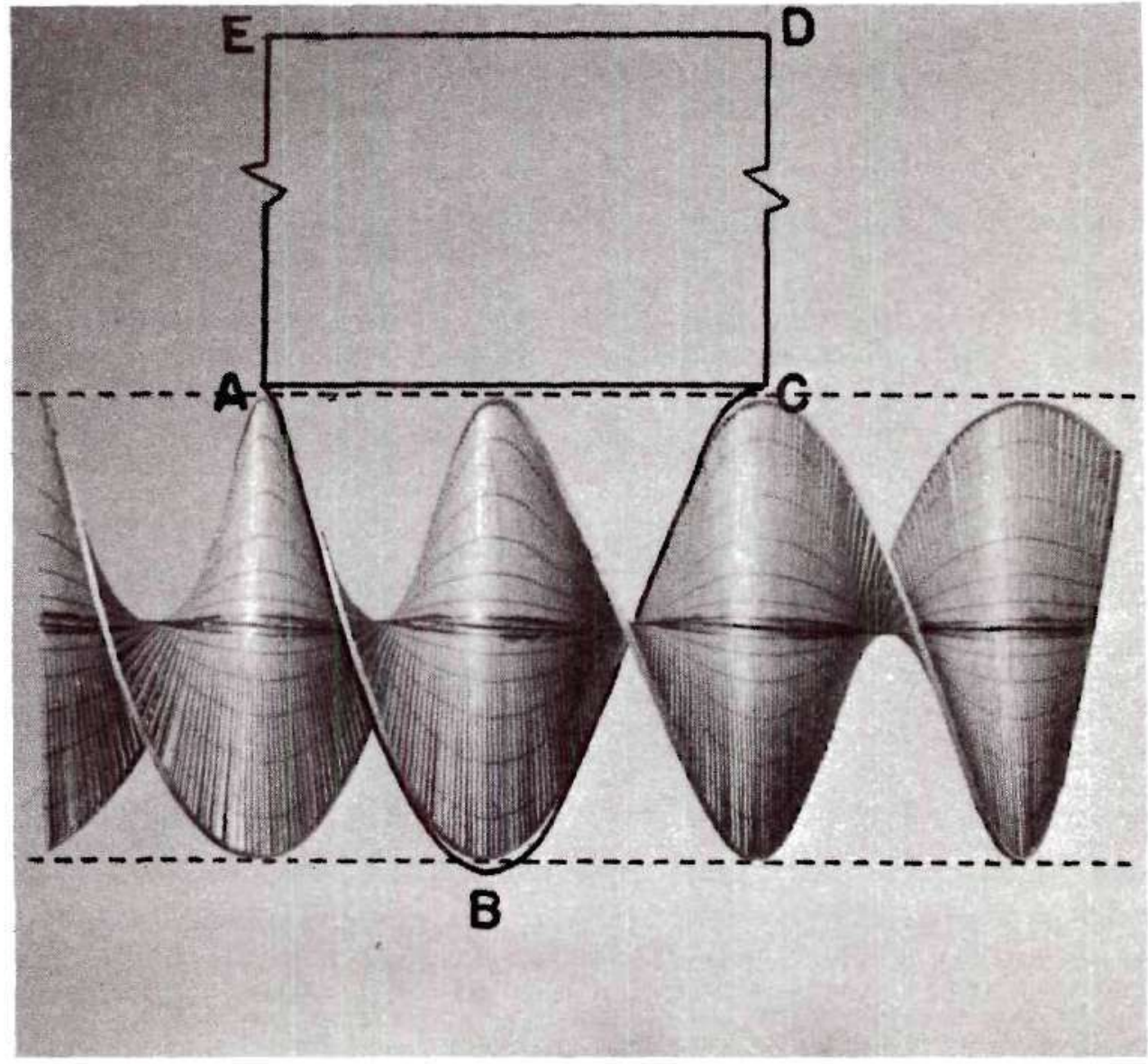

Fig. 5. Ultimate Wake System Showing Paths of Iine Integrals. 
the induend velcuity is zero along the radial lines $\overline{\mathrm{CD}}$ and $\overline{\mathrm{EA}}$. If $\overline{\mathrm{DF}}$ is allowed to apr roach infinity then $u_{z}$ allons $\overline{D E}$ is zero. Since no vorticit is enclus , by the path

$$
\int_{C}^{A} u_{z} d z=0
$$

The $n$, recing o the previous integration and subject to the condition of identical filament pitch angle along the intersection line, this result roguires that $u_{g_{0}}$ be zero. Thus, the induced velocity must bo nurmal to the inner helical sheets. These two coexistent conditions What filament piteh and normal induced velocity characterize the lightly loaded conditions as discussed by Gray ${ }^{4}$.

The morc cheil condition of load requires only that the filaments of the hounlary sheet all cross the intersection line at the sam pitch ansle so that a relative motion between the inner and outer civstoms ma: exist. Thus, the path $\overline{\text { EFGHF }}$ in Fig. 3 will enclose vorititi and no information will be obtained from the integral for is thivill loaded ductid fan. However, the requirements on $u_{\xi}$ still uppl, inside the walce, and for the apparent rigid motion of the inner he-heal sleets $u_{5}$ musl be proportional to $\cos \varphi$. That is, from the previons results ard the velocity diagram of Fig. 4

$$
\begin{aligned}
& u_{\xi}=u_{\xi} \sin \theta \\
& u_{\zeta}=w \cos c
\end{aligned}
$$


A geometry an motion must now be established for the boundary sheet which will mailtain helical symmetry of vortex strength distribution ani which will permit the boundary shoet to have an axial motion relative to the inner sheets. Further, the disturbance velocities must be zero outside the wak in order that the flow be irrotational there. This may bo showr by considexing the line integral about $\overline{A C D E A}$ as shown in 4 . 5. $\overline{\mathrm{EA}}$ and $\overline{C D}$ are radial lines along which $u_{r}$ must be zero by nulical symmetry. $\overline{E D}$ lies at $r=\infty$ so that $u_{z}$ is zero along this path. sinee the path rroless no vorticity the integral of $u_{z}$ along $\overline{\mathrm{AC}}$ must. a) zo. For this to occur either $u_{z}$ is identically zero or $u_{z}$ is part positive ant part nocative. From considerations of continuity, For whe latter endition to occur the flow must form closed streamlines within the area wint by the path of integration. However, integration of' velocit, along th so closer streamlin would yield finite values of circulation. Thus, a contradiction is arrived at in terms of the irrotationality of the flow so that $u_{z}$ must be zero along $\overline{A C}$. Another Line integral is taken along $\overline{\mathrm{ABCDE} A}$. For the helical portion of the path the velocity $u_{\xi}$ must be constant due to helical syrmetry. Since the vclocities along the other branches of the path are zero and no net: vorticity is cnclosed, $u_{\xi}$ must be identically zero along $\overline{\mathrm{ABC}}$. The path $\overline{A B C}$ may be shifted alone the z-axis to any axial position with no change in the results. The path is closed by joining the helical part to the rajial parts along the z-path $\overline{\mathrm{AC}}$. Thus with $u_{\xi}, u_{z}$ and $u_{r}$ all idontically zero on the outer surface it may be concluded that all. induced velocities are zero on the outer surface of the wake boundity. This results may be extonder to include the 
entire region oulside the wake by allowing the paths of integration to expand radial I.,

The strength " a vortex sheet is equal to the discontinuily in the velocity components as the sheet is crossed and the motion of the sheet along the line of discontinuity is equal to the mean value of the velocity $+1 R_{\text {- }}$ and $R_{+}$. At $r=R_{-}$, the indueed velocity at the inner surface of the boundary sheet is given by the components

$$
\begin{aligned}
& u_{\xi}=u_{\xi_{0}} \sin \omega_{R} \\
& u_{\zeta}=w \cos \varphi
\end{aligned}
$$

whiln the induced velocity a $\mathrm{R}_{+}$is zero. Thus at the line of intorsection with an imer holical sheet the oundary vortex sheel strength is givou t:

$$
y\left(r_{\beta}\right)-\left(u_{5} \sin ^{2} \varphi_{R}+w^{2} \cdot \cos ^{2} \omega_{R}\right)^{\frac{1}{2}}
$$

The velocity of the filaments of the boundary sheet as they cross the intersection lin must bo in the direction of the induced velocity at: $\mathrm{R}_{\text {_ }}$ and normal to the filament direction; that is

$$
u_{S_{B}}-\frac{1}{2}\left(u_{\xi_{0}}^{2} \sin ^{2} \varphi_{R}+w^{2} \cos ^{2} \theta_{R}\right)^{\frac{1}{2}}
$$

The filamerts of the bounary vorfox sheet must ther all eross the Iines of intersection between the inner surew surface and the cylintrical boundary at a constant angle $\varphi_{\mathrm{B}}$ not equal to $\varphi_{\mathrm{R}}$. This anclo may be deturnined from the light speed $\left(V_{\infty}\right)$, the blade rotational 
speed $(\Omega R)$ and the total disturbance velocity of equation (1). The two vortex systems are related through equations (1) and (2) but only along their linos of intersection. On the wake boundary between theso helical intersection lines, the filament density or sheet strength and the filament pitch angle will vary with the helical coordinate $\zeta$. The boundary sheet serves several basic purposes. First, it must cancel the radial velocity field at the boundary associated with the inner helical sheets. Second, it must accommodate the discontinuity in velocity as the boundary sheet is crossed. Third, it must not induce radial velocities, and hence radial distortions, at the inner sheet surfaces. Its remaining function is to preserve, in conjunction with the flow fields associated with the inner sheets, the apparent rigid axial motion of the inner sheets and to cancel the sum of vorticity of the inner sheets. The first and second requirements, along with the rigid axial motion constraint and the constraint on net vorticity, are satisfied by the strength distribution and the filament geometry both as yet unknown. The third condition may be automatically satisfied by a strength distribution and geometry that are symmetrical about the lines of intersection. This is simply a restatement of the helical. symmetry requirements on the vortex systems.

Having to solve for both the strength distribution and the filament feometry of the boundary vortex sheet presents considerable difficulty and implies the need for some kind of iterative procedure for locating the compatible strengths and positions of the boundary sheet filaments. This difficulty may be eliminated through a consideration of the implications of the helical symmetry requirements. 
It has been establishod that the boundary sheet strength and filament pi.tch angle are ronstent along an intersection line. At an arbitrary Jistance $\Delta z$ below this line, the strength and filament pitch angle will be different. However, at any other angular position at the same $\Delta z$ below the intersection line the pitch angle and strength must aiffer from the values at the intersection line by the same amounts due to the helical. symmetry requirement. That is, at a z-position between intersection lines it is possible to change the sheet strength and filament pitch angle only through the addition of an infinitesimal strength vortex filament at the pitch angle $\varphi_{R}$. Thus, it is possible to replace the boundary sheet by two simpler systems whose combine effects satisfy all of the conditions mentioned above.

The first of these sheets is a uniform sheet of helical vortex filaments having constant density $y\left(\zeta_{B}\right)$ and constant pitch angle $\varphi_{B}$ as shown in Fig. 6a. This sheet satisfies the required compatibility conditions as previously discussed. The second is a cylindrical sheet of helical filaments of varyine ant unknown strength but having a corstant and known pitch angle $\varphi_{\mathrm{R}}$ as shown in Fig. 6b. This sheet must have zero strength along the lines of intersection in order to proserve the compatibility established by the first sheet. It must have a symmetrical strength distribution about these lines and the lines midway between adjacent intersection lines and must cancel the radial velocities at the cylindrical boundary associated with the inner sheets. Superposition of the two sheets, as shown in Fig. 6c, must maintain the apparent rigid axial motion of the inner sheets in 


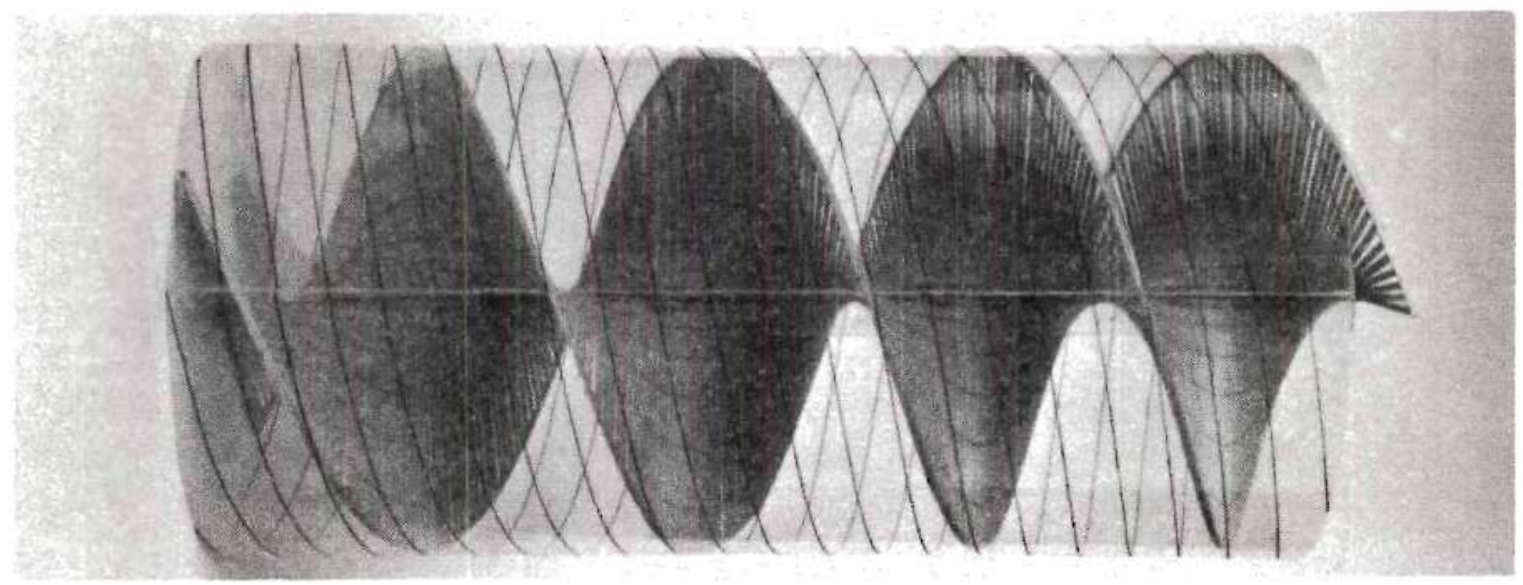

(a) Bourdary sheet of Uniform Strength.

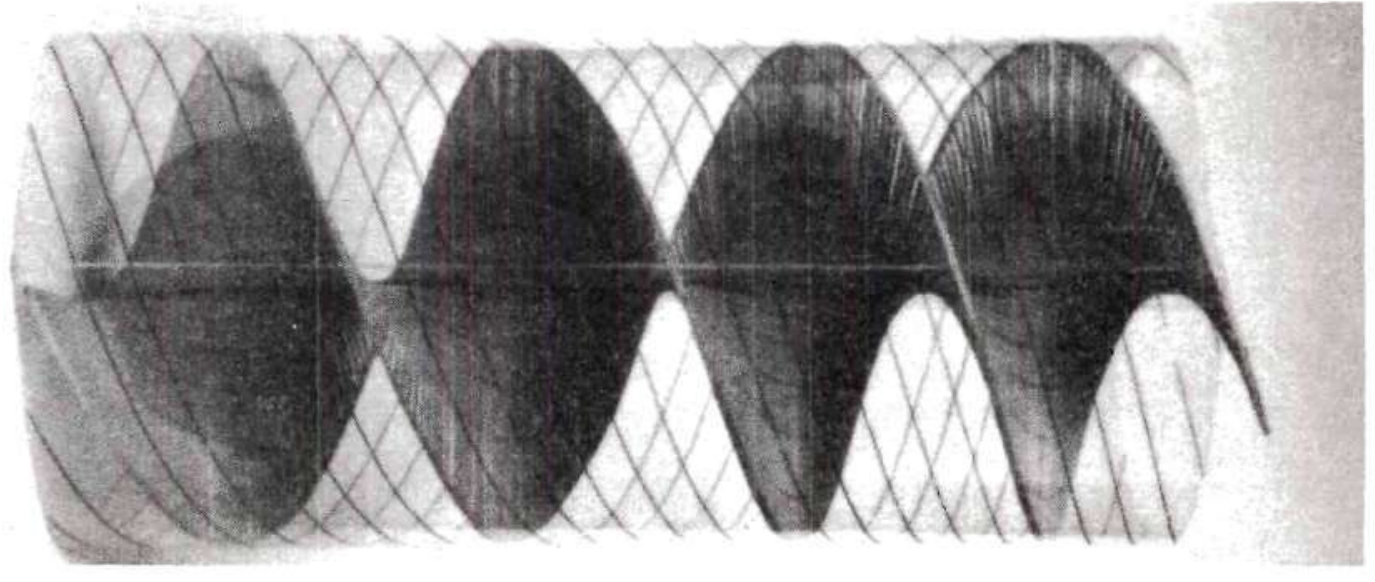

(b) Boundary sheet of Varying Strength.

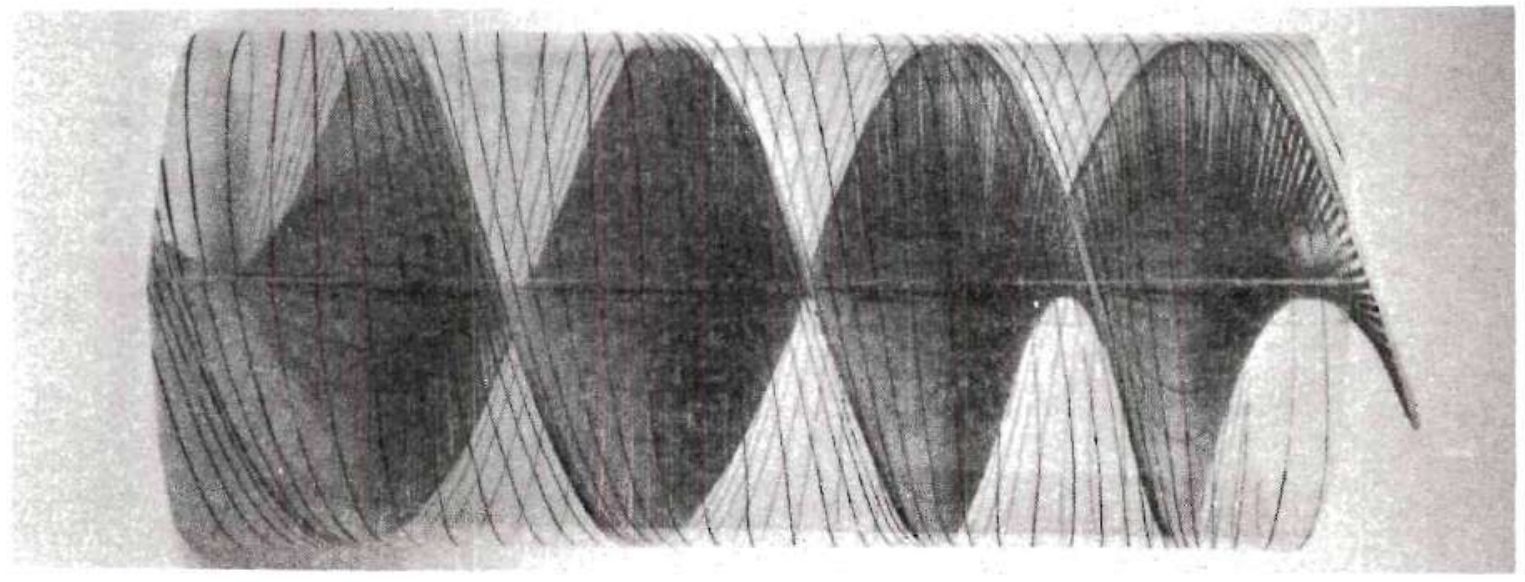

(e) Result of Superposition.

Fig. 6. Concept of Ultimate Wake Vortex System. 
conjunction with the flow fields associated with those sheets, and must satisfy the net vorticity constraint. These are the conditions which will be placed on the solution.

It should be noted that to an observer fixed on an inner helical sheet the boundary vortex sheet (consisting of the superposition of the uniform boundary sheet and the non-uniform boundary sheet) appears in terms of the local strength distribution and geometry to be fixed relative to the inner sheet. Although the boundary sheet is actually slipping forward relative to the inner sheets, the observer sees the same local vortex sheet strength at a given point on the boundary at any instant while the identity of the filaments at the point is constantIy changing.

Now consider the geometry and motion of the uniform boundary sheet relative to the last outboard filament of an adjacent inner: helical vortex sheet. The velocity diagram of Fig. 7 illustrates the relationship between the velocities associated with the two systems according to the compatibility condition expressed in equations (I) and (2). From Fig. 7

$$
\begin{aligned}
& \lambda=\tan \varphi_{R}=\frac{V_{\infty}+w}{\Omega R} \\
& \lambda_{B}=\tan \varphi_{B}=\frac{V_{\infty}+w_{B}}{\Omega R}
\end{aligned}
$$

where

$$
w_{B}=\frac{1}{2}\left(u_{\xi_{0}}^{2} \sin ^{2} \varphi_{R}+w^{2} \cos ^{2} \varphi_{R}\right)^{\frac{1}{2}} \sec \varphi_{B}
$$




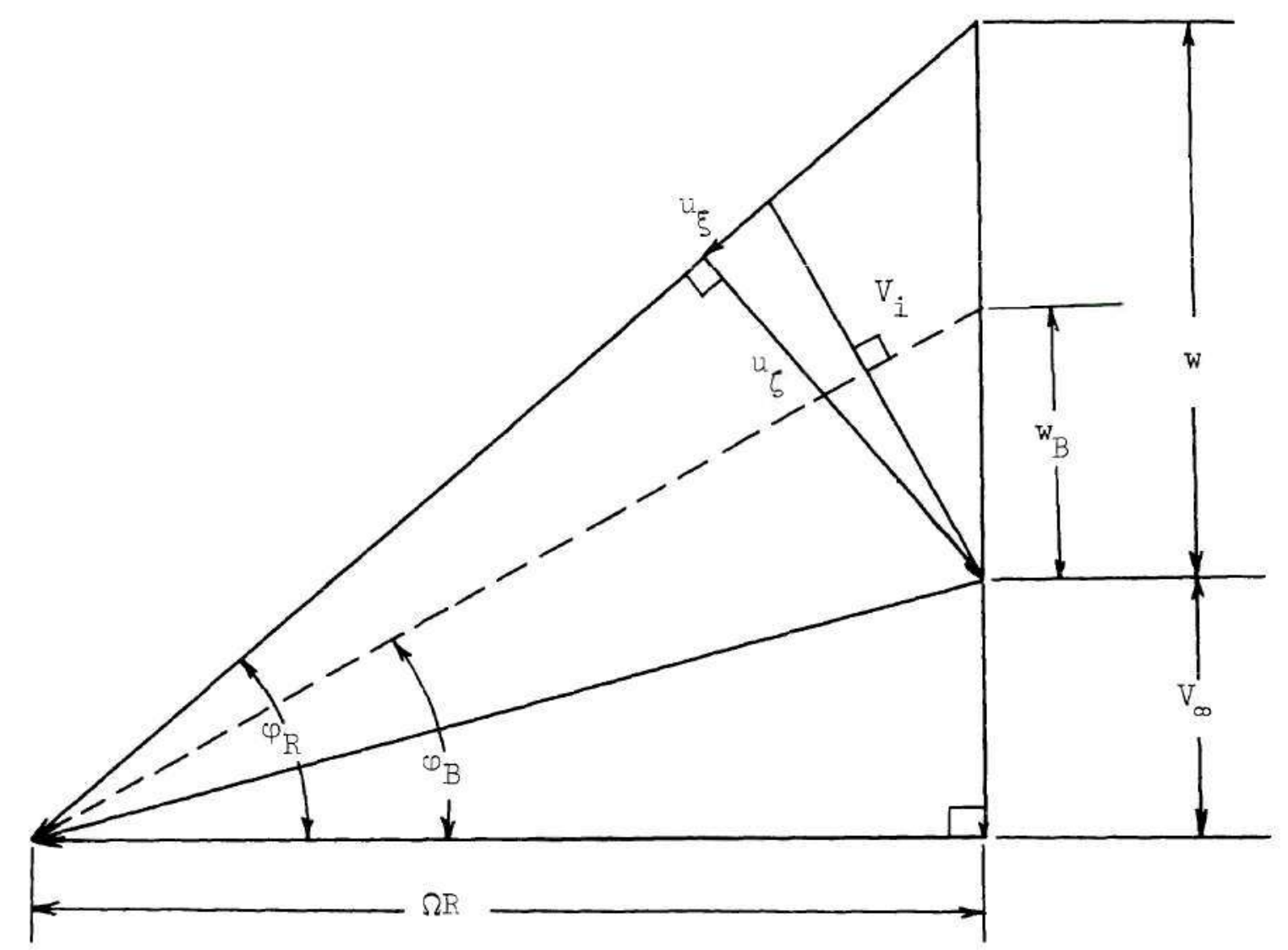

Figure 7. Velocity Diagram with respect to the Rotating Blades of the Outermost Filament of the Inner Vortex Sheet and at the Adjacent Point of the Boundary Sheet. 
From the same figure

$$
\frac{u_{\xi_{0}} \sin \varphi_{R}}{w \cos \varphi_{R}}=\tan \left(o_{R}-\varphi_{B}\right)
$$

Combining equation (4) and (5) to eliminate $u_{\xi_{0}}$ and substituting for the functions of aquations (3) yields

$$
\lambda_{B}=\lambda-\bar{w}\left[1+\frac{1}{2} \frac{1+\lambda_{B}^{2}}{1+\lambda \lambda_{B}}\right]
$$

where $\bar{w}=w / \Omega R, i \leq \overline{\mathrm{y}} \leq \lambda$. This result shows that, ior any choice of $\lambda$ and $\bar{w}, \lambda_{B}$ can be uniquely determined. It should be noted that for vanishingly small values of $\mathrm{w}, \lambda_{\mathrm{B}}$ approaches $\lambda$ which is in agreement with the lightly loadod case. Further, there would seem to be no simple redefinition of $\lambda$ which would reduce the solutions to a single case for all. values of $\overline{\mathrm{w}}$. This is in agreement with ulu arlier analyses of Gray ${ }^{4}$.

Solving the last expression for the pitch of the uniform boundary sheet yilids

$$
\lambda_{B}=\lambda-\frac{1+\lambda^{2}}{2 \lambda-\overline{\mathrm{w}}}+\left[\left(\lambda-\frac{1+\lambda^{2}}{2 \lambda-\overline{\mathrm{w}}}\right)^{3}+1\right]^{\frac{1}{2}}
$$

Since $\omega_{B}=\tan ^{-1} \lambda_{B}$, the value of $\gamma\left(\zeta_{B}\right)$ is known according to

$$
\gamma\left(\zeta_{B}\right)=w \cos \varphi_{R} \sec \left(\omega_{K}-{ }_{B}\right) \cdot
$$

The ultimate wake vortex system of the heavily loaded ducted fan is thus defined in terms of the inner helical cheets of Fig. 5 (one for 
each blade) having unknown strength but known geometry, a vortex sheet of uniform strength $\gamma\left(\zeta_{B}\right)$ and constant filament pitch angle $\varphi_{B}$ lying on the cylindrical wake boundary as shown in Fig. 6a, and a sheet of varying strength but constant filament pitch angle $\varphi_{R}$ also lying on the cylindrical wake boundary as shown in Fig. $6 \mathrm{~b}$. For a given blade number $b$, pitch $\lambda$, and loading parameter $\bar{w}$, the motion and geometry of the system are determined along with the strength of the uniform boundary sheet. The solution of this model for the unknown strength distributions of the inner sheets and the non-uniform boundary sheet proceeds directly through the application of the Biot-Savart equation. 
CHAPTER III

\section{ANALYSIS AND SOLUTION OF THE WAKE MODEL}

From the discussion of the preceding chapter, the geometry and motion of the ultimate wake vortex system of infinite length are known. The problem is now to find the distribution of vorticity in the wake which will satisfy the velocity boundary conditions implied by this geometry and motion. In this analysis, the Biot-Savart equation supplies the required relationship between the geometry, motion, and vortex sheet strengths. For an elemental length of an arbitrary vortex filament

$$
d v_{i}=\frac{\gamma}{4 \pi} \frac{\cos \beta d s^{\prime}}{\bar{P}^{2}}
$$

The integral relations for the velocity components in Cartesian coordinates are given by Lamb ${ }^{12}$ and are repeated here for a single finite strength vortex filament.

$$
\begin{aligned}
& \Delta u_{x}=\frac{y}{4 \pi} \int\left(\frac{d y^{\prime}}{d s^{\prime}} \frac{z-z^{\prime}}{\bar{P}}-\frac{d z^{\prime}}{d s^{\prime}} \frac{y-y^{\prime}}{\bar{P}}\right) \frac{d s^{\prime}}{\bar{P}^{2}} \\
& \Delta u_{y}=\frac{y}{4 \pi} \int\left(\frac{d z^{\prime}}{d s^{\prime}} \frac{x-x^{\prime}}{\bar{P}}-\frac{d x^{\prime}}{d s^{\prime}} \frac{z-z^{\prime}}{\bar{P}}\right) \frac{d s^{\prime}}{\bar{P}^{2}} \\
& \Delta u_{z}=\frac{y}{4 \pi} \int\left(\frac{d x^{\prime}}{d s^{\prime}} \frac{y-y^{\prime}}{\bar{P}}-\frac{d y^{\prime}}{d s^{\prime}} \frac{x-x^{\prime}}{\bar{P}}\right) \frac{d s^{\prime}}{\bar{P}^{2}}
\end{aligned}
$$


A vortex sheet of the wake system is divided into a number of equal width strips. These strips are replaced by vortex filaments of finite but unknown strengths lying on the centerlines of the strips. The strength of the filament must be equal to the integral of the sheet strength across the strip width. For purposes of numerical calculation, an adequate representation of the vortex sheet is achieved by placing a filament along the centerline of its corresponding strip, provided the strip width is sufficiently small compared to a characteristic sheet width.

The integrals for the velocity components associated with the finite strength helical vortex filaments introduced in this manner are more conveninntly expressed in polar coordinates so that the following transformation is made. (See Fig. 2.)

$$
\begin{aligned}
x^{\prime} & =r^{\prime} \cos \Psi^{\prime} \\
x & =r \cos \Psi \\
y^{\prime} & =r^{\prime} \sin \Psi^{\prime} \\
y & =r \sin \Psi \\
z^{\prime} & =z_{0}^{\prime}+r^{\prime} \Psi^{\prime} \tan \varphi^{\prime} \\
z & =z^{\prime} \\
s^{\prime} & =r^{\prime} \Psi^{\prime} \sec \varphi^{\prime}
\end{aligned}
$$

Employing the transformation yields 


$$
\begin{aligned}
& u_{x}=-\frac{y}{\pi} \int\left[r^{\prime} \tan \varphi^{\prime}\left(r \sin \psi-r^{\prime} \sin \Psi^{\prime}\right)\right. \\
& \left.-r^{\prime} \cos \Psi^{\prime}\left(z-z_{0}^{\prime}-r^{\prime} \Psi^{\prime} \tan \varphi^{\prime}\right)\right] \frac{d \Psi^{\prime}}{\overline{\mathrm{P}}^{3}} \\
& u_{y}=\frac{y}{4 \pi} \int\left[r^{\prime} \tan \varphi^{\prime}\left(r \cos \Psi-y^{\prime} \cos \Psi^{\prime}\right)\right. \\
& \left.+r^{\prime} \sin \Psi^{\prime}\left(z-z_{0}^{\prime}-r^{\prime} \Psi^{\prime} \tan 0^{\prime}\right)\right] \frac{d \Psi^{\prime}}{\overline{\mathrm{P}}^{3}} \\
& u_{z}-\frac{Y}{4 \pi} j\left[r^{\prime 2}-r r^{\prime} \cos \left(\Psi^{\prime}-\Psi\right)\right] \frac{1 \Psi^{\prime}}{\bar{p}^{3}}
\end{aligned}
$$

wherc

$$
\vec{p}^{2}=r^{2}+r^{\prime}-r^{\prime} \cos \left(\Psi^{\prime}-\Psi\right)+\left(z-z_{0}^{\prime}-r^{\prime} \Psi^{\prime} \tan \varphi^{\prime}\right)^{2}
$$

Thi boundary conditions are more onveniently expressed in terms of the velocity components along the vortex sheets and perpondicular to the shects. Thus

$$
\begin{aligned}
& u_{y^{r}}=u_{x} \cos \Psi+u_{y} \sin \Psi \\
& u_{\xi}=\left(u_{y} \cos \Psi-u_{x} \sin \Psi\right) \cos \varphi+u_{z} \sin \varphi \\
& u_{\zeta}=u_{z} \cos \varphi-\left(u_{y} \cos \Psi-u_{x} \sin \Psi\right) \sin \varphi
\end{aligned}
$$

The integrals for hes velorities due to a single finite strength iliamerit become 


$$
\begin{aligned}
& \Delta u_{r}=\frac{\gamma}{4 \pi} \int_{-\infty}^{\infty}\left[r^{\prime 2} \tan \varphi^{\prime} \sin \left(\Psi^{\prime}-\Psi\right)\right. \\
& \left.+r^{\prime}\left[z-z_{0}^{\prime}-r^{\prime} \Psi^{\prime} \tan \varphi^{\prime}\right] \cos \left(\Psi^{\prime}-\Psi\right)\right] \frac{d \Psi^{\prime}}{\bar{p}^{3}} \\
& \Delta u_{\xi}=\frac{\gamma}{4 \pi} \int_{-\infty}^{\infty}\left[\left\{r^{\prime} \tan \phi^{\prime}\left[r-r^{\prime} \cos \left(\Psi^{\prime}-\Psi\right)\right]\right.\right. \\
& \left.+r^{\prime}\left[z-z_{0}^{\prime}-r^{\prime} \Psi^{\prime} \tan \omega^{\prime}\right] \sin \left(\Psi^{\prime}-\Psi\right)\right\} \cos \varphi \\
& \left.+\left\{r^{\prime 2}-r r^{\prime} \cos \left(\Psi^{\prime}-\Psi\right)\right\} \sin \varphi\right] \frac{\mathrm{d} \Psi^{\prime}}{\overline{\mathrm{p}}^{3}} \\
& \Delta u_{\zeta}=\frac{y}{4 \pi} \int_{-\infty}^{\infty}\left[\left\{r^{\prime 2}-r r^{\prime} \cos \left(\Psi^{\prime}-\Psi^{\prime}\right)\right\} \cos \varphi\right. \\
& -\left\{r^{\prime} \tan \varphi^{\prime}\left[r-r^{\prime} \cos \left(\Psi^{\prime}-\Psi\right)\right]\right. \\
& \left.\left.+r^{\prime}\left[z-z_{0}^{\prime}-r^{\prime} \Psi^{\prime} \tan \varphi^{\prime}\right] \sin \left(\Psi^{\prime}-\Psi\right)\right\} \sin \varphi\right] \frac{\mathrm{d} \Psi^{\prime}}{\overline{\mathrm{P}}^{3}}
\end{aligned}
$$

where the limits on the integrals refer to the infinite extent of the ultimate wake.

Now, non-imensionalizing these equations with $\mathrm{w}$ and $\mathrm{R}$, and employing the helical relation

$$
r \tan \varphi=r^{\prime} \tan \varphi^{\prime}=R \tan \varphi_{\mathrm{R}},
$$

the elemental velocities associated with one filament become 


$$
\begin{aligned}
& \frac{\Delta u_{r}}{w}=\frac{\gamma}{4 \pi R w} \int_{-\infty}^{\infty}\left[\bar{r}^{\prime} \tan \varphi_{R} \sin \left(\Psi^{\prime}-\Psi\right)\right. \\
& \left.+\bar{r}^{\prime}\left[\bar{z}-\bar{z}_{\circ}^{\prime}-\Psi^{\prime} \tan \varphi_{R}\right] \cos \left(\Psi^{\prime}-\psi\right)\right] \frac{d \psi^{\prime}}{\bar{p}^{3}} \\
& \frac{\Delta u_{\xi}}{\mathrm{w}}=\frac{\gamma \cos \varphi}{4 \pi R \mathrm{~W}} \int_{-\infty}^{\infty}\left[\bar{r}^{\prime} \tan \varphi_{\mathrm{R}}\left(\frac{\bar{r}}{\bar{r}}+\frac{\bar{r}^{\prime}}{\bar{r}}-2 \cos \left(\Psi^{\prime}-\psi\right)\right)\right. \\
& \left.+\left[\bar{z}-\bar{z}_{0}^{\prime}-\Psi^{\prime} \tan \varphi_{R}\right] \sin \left(\Psi^{\prime}-\Psi\right)\right] \frac{d \Psi^{\prime}}{\bar{P}^{3}} \\
& \frac{\Delta u_{\zeta}}{w}=\frac{\gamma \cos \varphi}{4 \pi R w} \int_{-\infty}^{\infty}\left[\bar{r}^{\prime 2}-\bar{r} \bar{r}^{\prime} \cos \left(\Psi^{\prime}-\psi\right)\right. \\
& -\tan ^{2} \varphi_{R}\left[1-\frac{\bar{r}^{\prime}}{\bar{r}} \cos \left(\Psi^{\prime}-\Psi\right)\right] \\
& \left.-\frac{\bar{r}^{\prime}}{\overline{\bar{r}}} \sin \left(\Psi^{\prime}-\Psi\right) \tan \varphi_{\mathrm{R}}\left[\overline{\bar{z}}-\bar{z}_{0}^{\prime}-\Psi^{\prime} \tan \varphi_{\mathrm{R}}\right]\right] \frac{d \Psi^{\prime}}{\overline{\mathrm{P}}^{3}}
\end{aligned}
$$

where

$$
\begin{aligned}
& \overline{\mathrm{P}}^{2}=\bar{r}^{2}+\bar{r}^{\prime 2}-2{\bar{r} \bar{r}^{\prime}} \cos \left(\psi^{\prime}-\psi\right)+\left[\bar{z}-\bar{z}_{0}^{\prime}-\psi^{\prime} \tan \varphi_{R}\right]^{2} \\
& \varphi=\tan ^{-1}\left[\frac{1}{\overline{\bar{r}}} \tan \varphi_{R}\right]
\end{aligned}
$$

and $\bar{r}, \bar{r}^{\prime}, \bar{z}, \bar{z}_{0}^{\prime}$ are non-dimensionalized by $R$.

The boundary conditions may be written by summing the contributions of every filament of the system. They are, 
on the inner shect:

$$
\begin{aligned}
& \sum\left(\frac{\Delta u_{r}}{w}\right)=\cos \varphi \\
& \sum\left(\frac{\Delta u_{r}}{w}\right)=0
\end{aligned}
$$

and on the cylindrical boundary:

$$
\sum\left(\frac{\Delta u_{r}}{w}\right)=0
$$

The uniform boundary sheet induces no radial velocities anywhere in the ultimate wake and the non-uniform boundary sheet induces no radial velocities on an inner sheet due to its symmetry above and below a line of intersection. Examination of the integrand of equation (9) reveals that no radial velocities are induced at an inner helical sheet by the evenly spaced inner sheets themselves. Thus, equation (13) is satisfied identically. The condition on the cylindrical boundary, equation (14) involves only the inner sheets and the non-uniform boundary sheet. The condition of equation (12) involves all three systems. The remaining constraint requires that the sum of the strengths of all of the vortex filaments comprising the wake be zero. The boundary conditions may now be written on the inner sheet (from equation (12))

$$
\sum_{1}\left(\frac{\Delta u_{\zeta}}{w}\right)+\sum_{2}\left[\left(\frac{\Delta u_{\zeta}}{w}\right) A_{1}-\left(\frac{\Delta u_{\xi}}{w}\right) A_{2}\right]+\sum_{3}\left(\frac{\Delta u_{\zeta}}{w}\right)=\cos \varphi
$$

on the cylinarical boundary (from equation (14)) 


$$
\sum_{1}\left(\frac{\Delta u_{r}}{w}\right)+\sum_{3}\left(\frac{\Delta u_{r}}{w}\right)-0
$$

and

$$
\zeta_{1}\left(\frac{y}{\pi \pi R w}\right)+\sum_{2}\left(\frac{y}{4 \pi R w}\right)+\bar{\zeta}_{3}\left(\frac{\gamma}{4 \pi R w}\right)=0
$$

where $\sum_{1}$ refers to the inner sheets, $\sum_{2}$ refers to the uniform boundary sheet, $\sum_{2}$ refors to the non-uniform bouriary sheet anl

$$
\begin{gathered}
A_{1}=\cos \left(\varphi-\omega_{r_{B}}\right), \\
A_{2}=\sin \left(\varphi-\omega_{r_{B}}\right) \\
\tan r_{B}=\frac{1}{\bar{r}} \tan { }_{B} \text { and } \tan B=\frac{1}{r} \tan \oplus_{R} .
\end{gathered}
$$

For a fixed choice of $\lambda$ and $\bar{w}$, the conuributions of the uniform houmary shect to equitions (15) and (17) are fixed in terms of the isfort str ugth $\gamma\left(\zeta_{B}\right)$ and tho piteh anglc $0_{B}$. Thus the system of equatious wh be written more conveniently as

$$
\begin{gathered}
\sum_{1}\left(\frac{\Delta u_{C}}{w}\right)+\sum_{n_{3}}\left(\frac{\Delta u_{\zeta}}{w}\right)=\cos n-\sum_{2}\left\{\left(\frac{\Delta u_{c}}{w}\right) A_{1}-\left(\frac{\Delta u_{\xi}}{w}\right) A_{2}\right\} \\
\sum_{\eta}\left(\frac{\Delta u_{r}}{w}\right)+\sum_{3}\left(\frac{\Delta u_{r}}{w}\right)=0
\end{gathered}
$$


and

$$
\sum_{1}\left(\frac{y}{4 \pi R w}\right)+\sum_{3}\left(\frac{y}{4 \pi R w}\right)=-\sum_{c}\left(\frac{y}{4 \pi R w}\right)
$$

Equation (18) can be evaluated at a number of control points on an inner sheet. These points are placed between the filaments comprising the stoct, and are equal in number to the number of filaments on the sheet (the final point being placed at $r=\mathrm{R}$ ). Equation (19) can be evaluated at control points on the cylindrical boundary between un intersection line and the point midway between intersection lines. The points are ain placed between filaments with one less point than Pilaments. Equalion (20) includes the filaments on one inner sheet, the rilaments on the wn-uniform boundary shert lying between an intersection line and he adjacent intersection line, and those filaments of the niform boundary sheet passirg through a line comecting two adjacent intersection lines. These filaments comprise a characteristic portion of the vortex wake, although the velocitios must be caleulated using all of the filaments of the wake system.

$$
\text { Now, the intagrals of equations (9) and (11) may be lefined }
$$

respictivoly as

$$
\begin{gathered}
I_{r}=\left(\frac{\Delta u_{r}}{w}\right) /\left(\frac{\gamma}{4 \pi R w}\right) \\
I_{\zeta}=\left(\frac{\Delta u_{\zeta}}{w}\right) /\left(\frac{\gamma}{4 \pi R w} \cos 0\right)
\end{gathered}
$$

and the s.rstem of equations may be written as follows: 
for the control points on an inner sheet

$$
\sum_{1} I_{\zeta}\left(\frac{\gamma}{4 \pi R w}\right)+\sum I_{\zeta}\left(\frac{\gamma}{4 \pi R w}\right)=1-\sum_{-2}\left\{\left(\frac{\Delta u_{c}}{w}\right) A_{1}-\left(\frac{\Delta u_{\xi}}{w}\right) A_{2}\right\} / \cos \varphi
$$

for the control points on the cylindrical boundary

$$
\sum_{1} I_{r}\left(\frac{\gamma}{4 \pi R w}\right)+\sum_{3} I_{r}\left(\frac{\gamma}{4 \pi R w}\right)=0
$$

and

$$
\sum_{1_{c}}\left(\frac{\gamma}{4 \pi R w}\right)+\sum_{3_{c}}\left(\frac{\gamma}{4 \pi R w}\right)=-\sum_{c}\left(\frac{\gamma}{4 \pi R w}\right)
$$

where

$$
\begin{aligned}
& \sum_{c} \text { is over the filaments of one inner sheet, } \\
& \sum_{3_{c}} \text { is over the filaments of a characteristic }
\end{aligned}
$$

and

$$
\begin{aligned}
& \sum_{2} \text { is ovar the filaments of a characteristic } \\
& \text { portion of the uniform boundary sheet. }
\end{aligned}
$$

With the equations in this form, the influence of $\overline{\mathrm{w}}$ is conf'ined to the right hand sides of the equations through the velocity field of the uniform boundary sheet, and the coefficients $I_{r}$ and $I_{\zeta}$ depend only on the shice for $\lambda$ (the pitch of the inner sheets) and $b$ (the number of blates). 
Owing to the simplicity of the uniform bouniary sheet, the velocity rield insine the wake associated wi, th lihis sheet has been evaluated explicity in terms of $\lambda$ and $\bar{w}$ in Appendix II. The result, is

$$
\begin{aligned}
& \frac{u_{z}}{w}=\frac{\gamma\left(\zeta_{B}\right)}{w} \cos D_{B} \\
& \frac{u_{\Psi}}{w}=\frac{u_{r}}{w}=0 .
\end{aligned}
$$

Using equation $(i), \frac{u_{z}}{w}$ mon be written as

$$
\frac{u_{z}}{\eta}=\cos \omega_{\mathrm{L}} \sec \left(\omega_{\mathrm{R}}-\omega_{B}\right) \cos \varphi_{\mathrm{B}}
$$

$1)^{\circ}$

$$
\frac{u}{w}=\frac{1}{I+\lambda \lambda_{B}} .
$$

Then, the right hand side of equation (23) becomes

$$
1-L\left\{\left(\frac{\Delta u_{\zeta}}{w}\right) A_{\lambda}-\left(\frac{\Delta u_{\xi}}{L}\right) A_{2}\right\} / \cos \varphi=1-\frac{1}{1+\lambda \lambda_{B}}
$$

Further, h. right hand side of equation (25) becomes

$$
-\sum_{L_{c}}\left(\frac{\gamma}{4 \pi R w}\right)=-\frac{\gamma\left(\zeta_{B}\right)}{4 \pi R w}\left(2 \pi R \lambda_{B} \cos \varphi_{B} / b\right)
$$

Since $2 \pi R \lambda_{1} / b$ is the longth of the line defineng the characteristic portion of the uniform boundary sheet and $\gamma\left(\zeta_{B}\right)$ is the sheet strensth 
per unit length. This

$$
-\sum_{c_{c}}\left(\frac{\gamma}{4 \pi R_{w}}\right)=-\frac{1}{2 b}\left(\frac{\lambda_{B}}{I+\lambda \lambda_{B}}\right) .
$$

Some rearrangemcnt. oi the terms of equations (5) and (27) yields

$$
\begin{aligned}
& 1-I /\left(1+\lambda \lambda_{B}\right)=\frac{\lambda^{2}}{1+\lambda^{2}}\left[1-\frac{1}{\lambda}\left(\frac{\lambda-\lambda_{B}}{1+\lambda \lambda_{B}}\right)\right] \\
& \left.-\sum_{3}\left(\frac{\gamma}{\pi \operatorname{li} w}\right)=\frac{-1}{2 b}\left(\frac{\lambda}{1+\lambda^{2}}\right)\left[1-\frac{1}{\lambda} \frac{\lambda-\lambda_{B}}{\lambda+\lambda \lambda_{B}}\right)\right]
\end{aligned}
$$

From quation (5)

$$
\frac{{ }_{\xi_{0}} \sin \varphi_{R}}{w \cos \omega_{R}}-\tan \left(\omega_{R}-{ }_{B}\right)
$$

m

$$
\frac{\mathrm{u}_{\mathrm{O}}}{\mathrm{w}}=\frac{1}{\lambda}\left(\frac{\lambda-\lambda_{\mathrm{B}}}{1+\lambda \lambda_{\mathrm{B}}}\right)
$$

Wh that, definine

$$
G=1-\frac{{ }^{u} \xi_{0}}{w}
$$

equations (28) aru (29) may ve rowritten as

$$
1-1 /\left(1+\lambda \lambda_{B}\right)=\mathrm{G} \frac{\lambda}{1+\lambda^{2}}
$$




$$
-\sum_{3}\left(\frac{\gamma}{4 \pi R w}\right)=-\frac{G}{2 b}\left(\frac{\lambda}{1+\lambda^{2}}\right)
$$

Thus, the system of equations can be written

at the inner sheet control points

$$
\sum_{1} I_{5}\left(\frac{\gamma}{4 \pi R w}\right)+\sum_{3} I_{\zeta}\left(\frac{\gamma}{4 \pi R w}\right)=G \frac{\lambda^{2}}{I+\lambda^{2}}
$$

at the cylindrical boundary control points

$$
\sum_{1} I_{r}\left(\frac{\gamma}{4 \pi R w}\right)+\sum_{3} I_{r}\left(\frac{\gamma}{4 \pi r w}\right)=0
$$

and

$$
\sum_{c}\left(\frac{\gamma}{4 \pi R w}\right)+\sum_{3_{c}}\left(\frac{\gamma}{4 \pi R w}\right)=-G \frac{I}{2 b}\left(\frac{\lambda}{I+\lambda^{2}}\right)
$$

Since the right hand side of every equation is multiplied by $G$ (or is zero) a new vortex filament strength can be defined as

$$
\bar{Y}=\frac{1}{G}\left(\frac{Y}{4 \pi R W}\right)
$$

and the system written

$$
\begin{aligned}
& \text { at the inner sheet control points } \\
& \sum_{1} I_{\zeta} \bar{v}+\sum_{3} I_{5} \bar{\gamma}=\frac{\lambda^{2}}{1+\lambda^{2}}
\end{aligned}
$$


at the cylindrical boundaiy cortrol points

$$
\begin{aligned}
& \sum_{1} I_{r} \bar{Y}+\sum_{3} I_{Y} \bar{Y}=0 \\
& \sum_{1_{c}} \bar{Y}+\sum_{3_{c}} \bar{Y}=-\frac{1}{2 b}\left(\frac{\lambda}{1+\lambda^{2}}\right)
\end{aligned}
$$

The system of equatiuns in this form does wot conlain the $\mathrm{w}$ parameter. so that a colution ma be obtained which may be scaled direct. Ly for and value of $\overline{\mathrm{w}}$. 'that is, the equations are solved for $G=1(\overline{\mathrm{w}}=(3)$ wh the wak vorincity distribution for any wiue of $\overline{\mathrm{W}}$ is obtained by miltiply in the rosult, by the appropriate value of $\mathrm{G}$. The bladr: bound vortex strength is foun at any radial stetion: by summing the filamoris of an inner sheet lying inboard of the radial station in the ultimate wake. The remainirg lementes of the solut ion, the strengths of the lilament; of the nort-uniform boundary sheet, are requiruit in the calculation of the power ant thrust. The evaluation of the coeficionis $I_{Y}$ and $I_{5}$ of the unknown lilament strengths, the posiflusing of the filaments and control points on the sheets, and the simultarcous solution of the system of linear pquations tor the unknown strencths ar all considered in some detail in chapter 
CHAPTER IV

CALCUIATION OF THRUST, TOWER, AND INUCED EIPICIEIVY

The solution or the distribution of veltirity in the ultimst

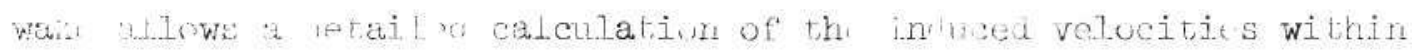
the wake. A knowl we of these velonitios, and hener of the momentum and ancre in the wake, leads to a straishtfu'ward calculasion of the thrust, power and efficiency.

\section{Thruat}

Following the t.lalysis of Theolorsen ${ }^{11}$.onsider a control volume enclosing the durte tar an! ins l]timal wak ds show whmatically in Fig. 8. Using tite monutum theorem the bisust oi th ducued fun may the found by considerit : the uverase preswar forces acting on the concrol surface and the averape il of momentum hroigh the surface. These averages are taken over time $\Delta t=2 \pi / \Omega$ an the integration is with respect to time, it $=\mathrm{z}_{\mathrm{z}} /\left(\mathrm{V}_{\mathrm{H}}+\mathrm{w}\right)$. Thus, from Fig.

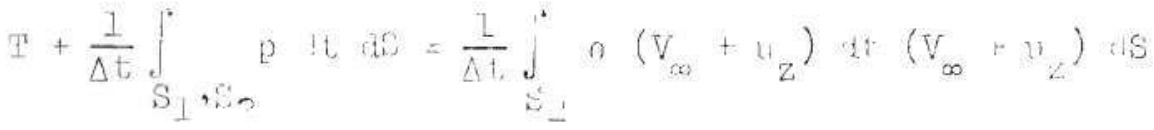

$$
\begin{aligned}
& -\frac{1}{\Delta t} \int_{0} \rho V_{\infty} \quad V_{t}, V_{i} \text { is }
\end{aligned}
$$

which can be written as 


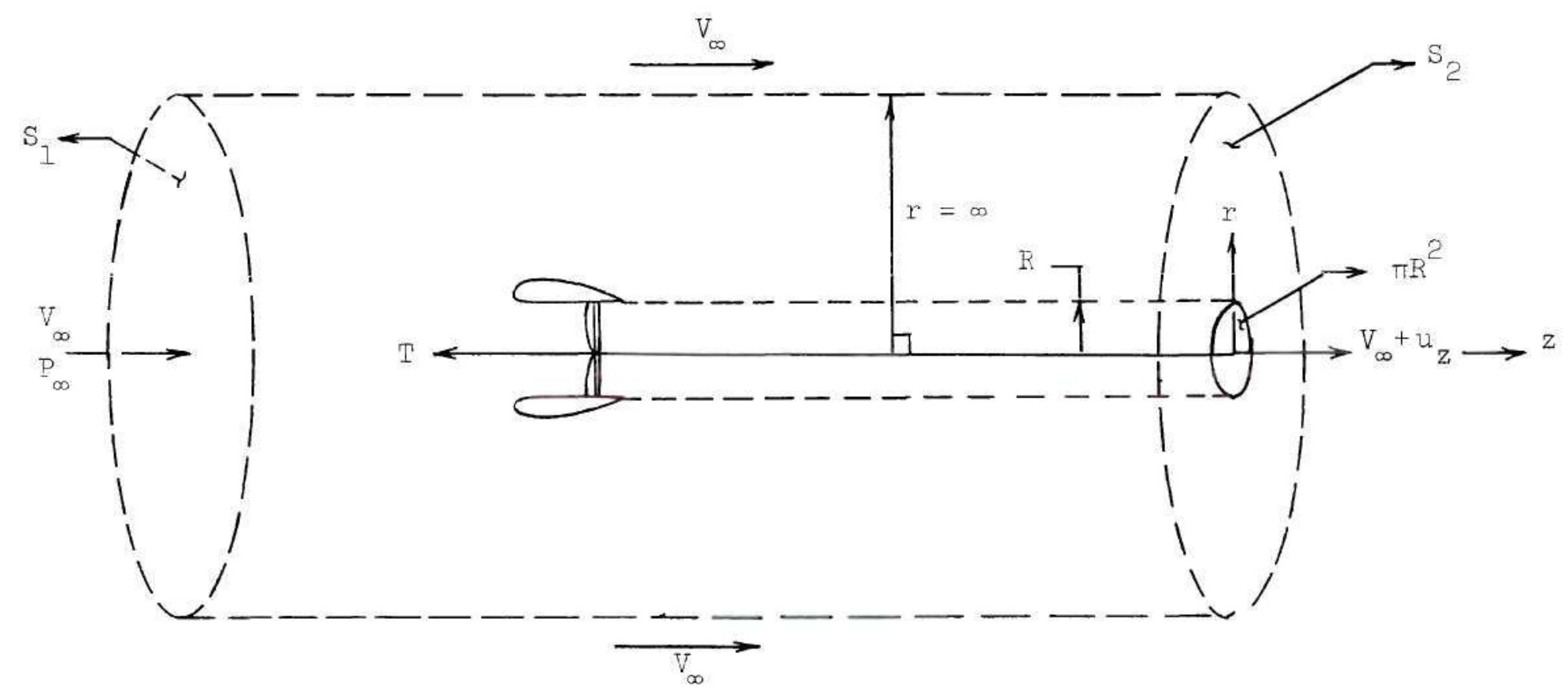

Figure 8. Control Volume Used in Determining the Thrust. 


$$
T+\frac{b \Omega}{2 \pi\left(V_{\infty}+w\right)} \int\left(p_{\infty}-p\right) d z d S=\frac{b \rho \rho}{2 \pi\left(V_{\infty}+w\right)} \int\left(V_{\infty} u_{z}+u_{z}^{2}\right) d z d S
$$

In order to integrate equation (34) the pressure term must first be eliminated by employing the equation of motion for an unsteady, incompressible, potential flow.

$$
\frac{\partial \Phi}{\partial t}+\frac{p}{p}+\frac{1}{2} v^{2}=f(t)
$$

Since there can be no induced velocities at an infinite radial distance from the wake axis

$$
\frac{\partial \Phi}{\partial t}+p / \rho+v^{2} / 2=p_{0} / \rho
$$

The unsteady term may be eliminated by considering the potential f'ield in a steady coordinate system such that

$$
\Phi(r, \Psi, z)=\Phi\left(r, \Psi_{0}+\Omega r t, z\right)
$$

Theri

$$
\frac{\partial \Phi}{\partial t}=\frac{\partial \Phi}{\partial \Psi} \frac{\partial \Psi}{\partial t}=u_{\Psi} \Omega r=x u_{\Psi} \Omega R
$$

Now $u_{\Psi}$ may be written as

$$
u_{\psi}=u_{\xi_{0}} \sin \varphi \cos \varphi-u_{\zeta} \sin \varphi
$$

and 


$$
u_{z}=u_{\xi_{0}} \sin ^{2} \varphi+u_{\zeta} \cos \varphi,
$$

Thus

$$
u_{5} \sin \theta=u_{z} \tan \theta-u_{0} \sin ^{2} \varphi \tan \theta .
$$

$u_{\psi}$ becomes

$$
u_{\psi}=\tan \varphi w(1-G)-u_{z} \tan \varphi
$$

and, since $\tan \varphi=\lambda / x=\left(\frac{V_{\infty}+w}{\Omega R}\right) / x, \frac{\partial \Phi}{\partial t}$ inside the wake becomes

$$
\frac{\partial \Phi}{\partial t}=-\left(V_{\infty}+w\right) u_{z}+w\left(V_{\infty}+w\right)(I-G)
$$

Then inside the wake equation (35) is

$$
p / 0+v^{2} / 2-\left(V_{\infty}+w\right) u_{z}+w\left(V_{\infty}+w\right)(1-G)=p_{o_{R_{-}}} / \rho
$$

The pressure must also be constrained by a static pressure balance at the wake boundary. Since all disturbances vanish outside the wake, the constraint becomes $p_{R_{-}}=p_{\infty}$ or

$$
p_{\infty}=p_{o_{-}}-\frac{1}{2} \rho v_{R_{-}}^{2}+\rho\left(V_{\infty}+w\right) u_{z_{R_{-}}}-\rho\left(V_{\infty}+w\right) w(I-G)
$$

Then using equation (36) to eliminate $\mathrm{p}_{\mathrm{O}_{\mathrm{R}}}$ yields 


$$
\left(p_{\infty}-p\right)=\frac{1}{2} \rho\left(v^{2}-v_{R_{-}}^{2}\right)-\rho\left(v_{\infty}+w\right)\left(u_{z}-u_{z_{-}}\right)
$$

where

$$
\begin{aligned}
& v^{2}=\left(v_{\infty}+u_{z}\right)^{2}+u_{r}^{2}+u_{\psi}^{2} \\
& \mathrm{~V}_{\mathrm{R}_{-}}^{2}=\left(\mathrm{v}_{\infty}+u_{\mathrm{z}_{-}}\right)^{2}+\psi_{\mathrm{R}_{-}} \\
& \text {Crabtitution of equation (37) into equ tion (34) vields } \\
& \uparrow=\frac{k \Omega \rho}{2 \pi\left(v_{\infty}+w\right)} \int_{\substack{\text { Volume } \\
\text { Inside } \\
\text { Wake }}}\left[v_{\infty} u_{z}+u_{z}^{2}-\frac{1}{2}\left(\left[v_{\infty}+u_{i}\right]^{2}\right.\right. \\
& \left.-\left[v_{\infty}+z_{R_{-}}\right]^{2}+u_{r}^{2}: u_{\psi}^{2}-u_{\psi_{R}}^{2}\right) \\
& \left.+\left(v_{\infty}+w\right)\left(u_{z}-u_{z_{R}}\right)\right] r d r d z d \Psi
\end{aligned}
$$

The limits of integrauion are taken over a chiracteristic volume cf i.h. waks such that

$$
\begin{gathered}
0 \leq z \leq 2 \pi R \lambda / b \\
0 \leq r \leq R \\
0 \leq \Psi \leq 2 \pi
\end{gathered}
$$

Then def'ining non-dimensional lengths as 


$$
\begin{gathered}
\vec{z}=z /(2 \pi R \lambda / b), \quad 0 \leq \bar{z} \leq 1 \\
\bar{r}=r / R, \quad 0 \leq \bar{r} \leq 1
\end{gathered}
$$

the thrust can be written as

$$
\begin{aligned}
& T=\frac{b \Omega \rho}{2 \pi\left(V_{\infty}+w\right)} R^{2}(2 \pi R / / b) \int_{0}^{l} \int_{0}^{l} \int_{0}^{2 \pi}\left[V_{\infty} u_{z}+u_{z}^{2}-\frac{1}{2}\left[\left(V_{\infty}+u_{z}\right)^{2}\right.\right. \\
& \left.\left.-\left(V_{\infty}+u_{z_{R_{-}}}\right)^{2}+u_{r}^{2}+u_{\psi}^{2}-u_{z_{R_{-}}^{2}}^{2}\right]+\left(V_{\infty}+w\right)\left(u_{z}-u_{z_{R}}\right)\right] \bar{r} d \bar{r} d \bar{z} d \Psi / 2 \pi
\end{aligned}
$$

Now, non-dimensionalizing the velocities according to $\bar{u}=u / w$ and $\overline{\mathrm{w}}=\mathrm{w} / \Omega \mathrm{R}$ yields

$$
\begin{aligned}
& T=2 \bar{w}^{2}(\Omega R)^{2} \int_{0}^{1} \int_{0}^{1} \int_{0}^{2 \pi}\left[\frac{1}{w} \frac{V_{\infty}}{\Omega R} \bar{u}_{z}+\bar{u}_{z}^{2}-\frac{1}{2}\left[\left(\frac{V_{\infty} / \Omega R}{\bar{w}}+\bar{u}_{z}\right)^{2}\right.\right. \\
& \left.-\left(\frac{V_{\infty} / \Omega R}{\bar{w}}+\bar{u}_{z_{R}}\right)^{2}+\bar{u}_{r}^{2}+\bar{u}_{\Psi}^{2}-\bar{u}_{\psi}\right] \\
& \left.+\left(\frac{V_{\infty} / \Omega R}{\bar{w}}+1\right)\left(\bar{u}_{z}-\bar{u}_{z_{-}}\right)\right] \bar{r} d \bar{r} d \bar{z} d \Psi / 2 T
\end{aligned}
$$

Noting that $\left(V_{\infty} / \Omega R\right)=(\lambda-\bar{w})$ and defining a thrust coefficient as

$$
C_{T}=T /\left[\rho(\Omega R)^{2} \pi R^{2}\right]
$$

the result may be reduced to 


$$
\begin{aligned}
& C_{T}=2 \bar{w}^{2} \int_{0}^{1} j_{0}^{1} \int_{0}^{2 \pi}\left[\frac{\lambda}{\bar{w}} \bar{u}_{z}+\bar{u}_{z}^{2}-\frac{1}{2}\left[\bar{u}_{z}^{2}+\bar{u}_{r}^{2}+\bar{u}_{\psi}^{2}\right]+\frac{1}{2}\left[\vec{u}_{z_{R}}^{2}+\bar{u}_{\psi_{R}}^{r}\right]\right. \\
& \left.-\vec{u}_{z_{R}}\right] \bar{r} \quad \vec{x} d \vec{z} d \psi / 2 \pi
\end{aligned}
$$

Some of the terms may be integrated imnediately as follows. Consiler a line integral within the waike as shown in Fig. 9. The intugral about the path $\overline{A P C D A}$ encloses those filaments she by a la toun 3 vortex botwen $\because=0$ and $\mathrm{x}=\mathrm{x}(\mathrm{x}-\overline{\mathrm{x}})$ so that the line intogr: i. $\quad \Gamma(x)$ and

$$
\int_{A}^{B} u_{r}+\int_{\Gamma}^{C} u_{z} d z+\int_{C}^{D} u_{r} d r+\int_{T}^{A} u_{z} d z=\Gamma(x)
$$

But rom the remiring on velocity discontinuity acrosis the shet, (for which $u_{r}$ of the filaments is zero)

$$
\int_{A}^{b} u_{r} d r=-\int_{C}^{D} u_{r} d t
$$

se that

$$
\int_{D}^{A} u_{z} d z-u_{\xi_{0}}(2 \pi R \lambda / b)=\Gamma(x)
$$

or.

$$
\int_{c}^{1} \bar{u}_{z} d \bar{z}=\frac{b \Gamma(x)}{2 \pi R w \lambda}+\bar{u}_{\xi_{0}}
$$




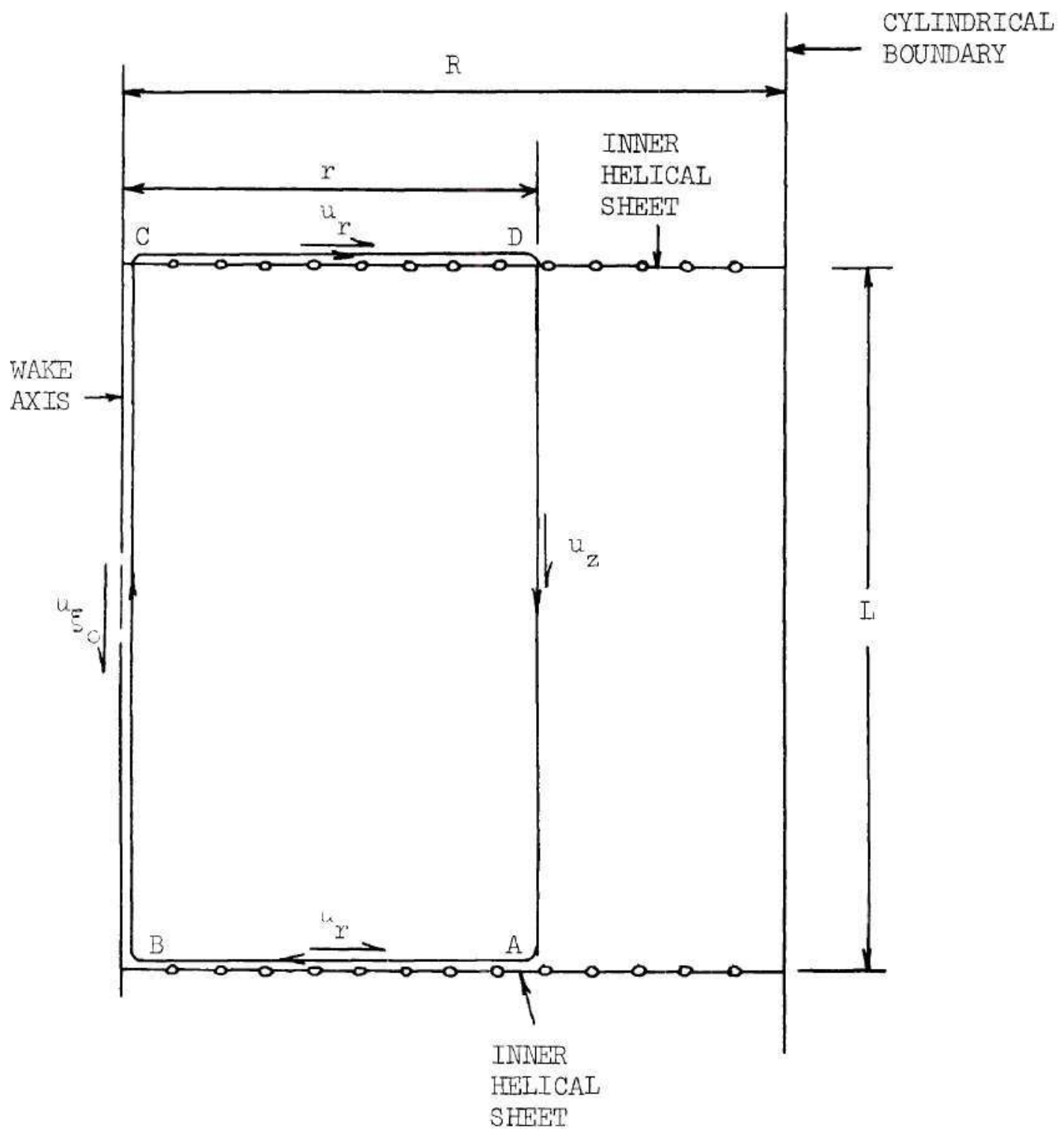

Pisur 9. A Path of Line Integration in the Ultimate Wake. 
Imploying the definition of Theodorsen for the non-dimensional blade bound vortex strength as

$$
K(x)=\frac{b \Gamma(x)}{2 \pi R w \lambda}
$$

yieIds

$$
\int_{0}^{1} \bar{u}_{z} d \bar{z}=K(x)+\bar{u}_{\xi_{0}}
$$

so that

$$
\begin{aligned}
\int_{0}^{1} \int_{0}^{1} \int_{0}^{2 \pi} \bar{u}_{z} \bar{x} d \bar{r} d \bar{z} d \Psi / 2 \pi & =\int_{0}^{1} \int_{0}^{2 \pi}\left[K(x)+\bar{u}_{\xi_{0}}\right] x d x d \Psi / 2 \pi \\
& =\frac{1}{2}\left[2 \int_{0}^{1} K(x) x d x+\bar{u}_{\xi_{0}}\right] .
\end{aligned}
$$

But the integral term is defined ${ }^{11}$ as $K$, the propeller mass coefficient,

$$
x=2 \int_{0}^{1} K(x) x d x
$$

Thus

$$
\int_{0}^{1} \int_{0}^{1} \int_{0}^{c \pi} \bar{u}_{z} \bar{r} d \bar{r} d \bar{z} d \Psi / 2 \pi=\frac{I}{2}\left[\kappa+\bar{u}_{\xi_{0}}\right]
$$

If $\bar{x}$ is taken as $\bar{r}=x=I$ then $u_{z}$ becomes $\bar{u}_{z_{R}}$ so that the integration of the last term of equation (39) becomes 


$$
\int_{0}^{1} \int_{0}^{1} \int_{0}^{2 \pi} \bar{u}_{z_{R_{-}}} \bar{r} d \bar{r} d \bar{z} d \Psi / 2 \pi=\frac{1}{2}\left[K(1)+\bar{u}_{\xi_{0}}\right]
$$

The remaining terms of the integral of equation (39), which must be evaluated numerically, may be defined as

$$
\begin{aligned}
& \epsilon_{z}=\int_{0}^{1} \int_{0}^{I} \int_{0}^{2 \pi} \bar{u}_{z}^{2} \bar{r} d \bar{r} d \bar{z} d \Psi / 2 \pi \\
& \epsilon_{r}=\int_{0}^{1} \int_{0}^{1} \int_{0}^{2 \pi} \bar{u}_{r}^{2} \bar{r} d \bar{r} d \bar{z} d \Psi / 2 \pi \\
& \epsilon_{\Psi}=\int_{0}^{1} \int_{0}^{1} \int_{0}^{2 \pi} \bar{u}_{\Psi}^{2} \bar{r} d \bar{r} d \bar{z} d \Psi / 2 \pi \\
& \epsilon_{R}=\int_{0}^{1} \int_{0}^{1} \int_{0}^{2 \pi}\left[\bar{u}_{z_{R}}^{2}+\bar{u}_{\Psi}^{2}\right] \bar{r} d \bar{r} d \bar{z} d \Psi / 2 \pi
\end{aligned}
$$

and $\epsilon=\epsilon_{z}+\epsilon_{r}+\epsilon_{\psi}$. Employing these definitions and equations (42) and (43), equation (39) becomes

$$
C_{T}=\overline{\mathrm{w}}^{2}\left[\frac{\lambda}{\overline{\mathrm{w}}} \mathcal{K}-\mathrm{K}(1)+\left(\frac{\lambda}{\overline{\mathrm{w}}}-1\right) \overline{\mathrm{u}}_{\xi_{0}}+2 \epsilon_{z}-\epsilon+\epsilon_{R_{-}}\right]
$$

It has been established that the flow field of the uniform boundary sheet is known and that the vortex sheet strength distributions, and hence the flow fields, of the sheets of varying strength need only 
be solved for tho ljghtly loaded condition. It is possible, then, to ealculate the thrust coefficient in terms or the $\overline{\mathrm{w}}=0$ wake solution and the scale factor G. Referring to equation (39) the integral terms can be modified as follows. The velocities are separated into those associated with the inner helical and non-uniform boundary sheets (variable strength, subscript $\mathrm{vs}_{\overline{\mathrm{w}}}$ ) and those associated with the uniform boundary sheet. The vs $\bar{w}_{\bar{w}}$ velocities can be scaled according to

$$
\overline{\mathrm{u}}_{\mathrm{vS_{ \overline { \textrm {w } } }}}=\mathrm{G} \overline{\mathrm{u}}_{\mathrm{vS}}=\mathrm{G} \overline{\mathrm{u}}_{\mathrm{VS}}
$$

Further

$$
K(x)=G K(x)_{\overline{\mathrm{w}}=0}=G K_{0}(x) \text { and } \kappa=G K_{0} \text {. From }
$$

equation (28) the velocity associated with the uniform boundary sheet is simply $\vec{u}_{z}=1-\frac{G \lambda^{2}}{1+\lambda^{2}}$ Cor all. $\vec{r}$. For simplicity in the following development $\int d\left(\right.$ vol.) will l be taken to represent $\int_{0}^{1} \int_{0}^{1} \int_{0}^{2 \pi} \bar{r} d \bar{r} d \bar{z} d \Psi / 2 \pi$.

The integrals in cquation (39) can then be written as

$$
\begin{aligned}
& \int \bar{u}_{z}^{2} a(\text { vol })=\int\left[G \bar{u}_{z_{\text {vs }}}+\left(1-\frac{G \lambda^{2}}{1+\lambda^{2}}\right)\right]^{2} d(\text { vol }) \\
& =G^{2} \int \vec{v}_{z_{v s}^{2}} a(\operatorname{vol})+2 G\left(I-\frac{G \lambda^{2}}{1+\lambda^{2}}\right) \int \vec{u}_{z_{v s}} d(\operatorname{vol}) \\
& +\frac{1}{2}\left(1-\frac{G \lambda^{2}}{1+\lambda^{2}}\right)^{2}, \\
& \int \bar{u}_{\psi}^{2} a(\text { vol })=G^{2} \int_{\psi_{\text {vs }}}^{2} d(\text { vol }),
\end{aligned}
$$




$$
\begin{aligned}
& \int \bar{u}_{r}^{2} a(\text { vol })=G^{2} \int \bar{u}_{r_{\text {vs }}}^{2} d(\text { vol }), \\
& \int \bar{u}_{z_{-}^{2}}^{2} d(\text { vol })=G^{2} \int \bar{u}_{z_{\text {vs }}} d(\text { vol })+2 G\left(1-\frac{G \lambda^{2}}{1+\lambda^{2}}\right) \int \bar{u}_{z_{\text {vs }}} d(\text { vol }) \\
& +\frac{1}{2}\left(1-\frac{G \lambda^{2}}{1+\lambda^{2}}\right)^{2}, \\
& \int \bar{u}_{\Psi_{-}} a(\operatorname{vol})=G^{2} \int_{v_{\mathrm{Vs}_{-}}} d(\operatorname{vol}) .
\end{aligned}
$$

From equation (42)

$$
\int \bar{u}_{z_{V S}} \partial(\operatorname{vol})=\frac{1}{2}\left[K_{0}-\frac{1}{1+\lambda^{2}}\right]
$$

and from equation (43)

$$
\int \bar{u}_{z_{\text {vs } F_{-}}} d(\text { vol })=\frac{1}{2}\left[K_{0}(I)-\frac{I}{I+\lambda^{2}}\right]
$$

Substitutine these results and the results of equations (42) and (43) into equation (39) yields

$$
\begin{aligned}
C_{T}=2 \bar{w}^{2}\left[\frac{1}{2} \frac{\lambda}{\bar{w}}\left(G \kappa_{0}+1-G\right)\right. \\
\\
\quad+\frac{1}{2}\left[G^{2} \int \vec{u}_{z_{V S}}^{2} a(v O I)+G\left(1-\frac{G \lambda^{2}}{1+\lambda^{2}}\right)\left(\kappa_{0}-\frac{1}{1+\lambda^{2}}\right)\right.
\end{aligned}
$$




$$
\begin{aligned}
& \left.+\frac{1}{2}\left(1-\frac{G \lambda^{2}}{1+\lambda^{2}}\right)^{2}\right]-\frac{1}{2} G^{2}\left[\int \bar{u}_{r_{\text {vs }}}^{2} d(\text { vol })+\int \bar{u}_{\psi}^{2} d(\text { vol })\right] \\
& +\frac{1}{2}\left[G^{2} \int \bar{u}_{v_{V s_{-}}^{2}}^{u_{R_{-}}} d(\text { vol })+G\left(1-\frac{G \lambda^{2}}{1+\lambda^{2}}\right)\left(K_{0}(1)-\frac{1}{1+\lambda^{2}}\right)\right. \\
& +\frac{1}{2}\left(1-\frac{G \lambda^{2}}{1+\lambda^{2}}\right)^{2} \\
& \left.\left.+G^{2} \int_{V_{V S_{R}}^{2}}^{2} d(\text { Vol })\right]-\frac{1}{2}\left(G K_{0}(I)+I-G\right)\right]
\end{aligned}
$$

The remaining integrals, which must be evaluated numerically, may be regrouped and defired as

$$
\epsilon_{u}=\int_{0}^{1} \int_{0}^{2 \pi}\left[\bar{u}_{z_{\text {VS }}}^{2}-\bar{u}_{r_{\text {VS }}}^{2}-\bar{u}_{\Psi}^{2}+\bar{u}_{z_{\text {vs }}}^{2}\right] \bar{r} d \bar{r} d \bar{z} d \Psi / 2 \pi
$$

Ising the definition of equation (45), the thrust coefficient may be written as

$$
\begin{aligned}
C_{T}=\overline{\mathrm{w}}^{2}\left[G\left(\frac{\lambda}{\overline{\mathrm{w}}}+1-\frac{G \lambda^{2}}{1+\lambda^{2}}\right) k_{0}-\frac{G^{2} \lambda^{2}}{1+\lambda^{2}} K_{0}(1)+G^{2} \epsilon_{0}\right. \\
\left.+(1-G)\left(\frac{\lambda}{\overline{\mathrm{w}}}+1-\frac{2 G \lambda^{2}}{1+\lambda^{2}}\right)-\left(1-\frac{G \lambda^{2}}{1+\lambda^{2}}\right)^{2}\right]
\end{aligned}
$$


where, for a given $b$ and $\lambda, k_{0}, K_{0}(I)$ and $\epsilon_{0}$ are calculated from the $\overline{\mathrm{w}}=0$ solution. Since $\mathrm{G}$ is calculated algebraically for a given $\lambda$ and $\overrightarrow{\mathrm{w}}$, equation (46) provides an entire family of values for $C_{T}$ whereas equation (44) provides only a single value.

\section{Power}

The ideal power required by the heavily loaded ducted fan may be determined through a consideration of the induced energy loss in the ultimate wake. Following Theodorsen ${ }^{11}$ the energy loss, E, is given by the methods of classical mechanics as

$E=\otimes \Omega-T V_{\infty}=\frac{b \Omega}{2 \pi\left(V_{\infty}+w\right)} \int\left[\frac{1}{2} \rho V_{\infty} v^{2}+\left(\frac{I}{2} \rho v^{2}+p-p_{\infty}\right) u_{z}\right] d(v o l)(47)$

where $Q$ is the torque of the fan and $v^{2}$ is the magnitude of the total induced velocity, $v^{2}=u_{z}^{2}+u_{r}^{2}+u_{\psi}^{2}$. Substituting from equation (37) for $\left(p-p_{\infty}\right)$, the energy loss becomes

$$
E=\frac{b \Omega p}{2 \pi\left(v_{\infty}+w\right)} \int\left[\frac{1}{2} v_{\infty} v^{2}+w u_{z}^{2}+u_{z}\left(\frac{1}{2} v_{R_{-}}^{2}-w u_{z_{-}}\right)\right] d(v 0 l)
$$

Then, defining a non-dimensional energy loss as

$$
e=E /\left[\rho(\Omega R)^{3} \pi R^{2}\right]
$$

and non-dimensionalizing velocities and lengths as was done for the thrust coefficient, e becomes

$e=2 \bar{w}^{3} \int_{0}^{1} \int_{0}^{1} \int_{0}^{2 \pi}\left[\bar{u}_{z}^{2}+\frac{1}{2}\left(\frac{\lambda}{\bar{w}}-1\right) \bar{v}^{2}+\frac{1}{2} \bar{u}_{z}\left(\bar{v}_{R_{-}}^{2}-\bar{u}_{z_{R_{-}}}\right)\right] \bar{r} d \bar{r} d \bar{z} d \Psi / 2 \pi$ 
Again, dividing the induced velocities into those associated with the uniform boundary sheet and those associated with the variable strength sheets, the expression for e can be calculated in terms of $G$ and the $\overline{\mathrm{w}}=0$ solution. All manipulations and integrations are similar to those performed in the thrust analysis. The result is given as

$$
\begin{aligned}
& e=\bar{w}^{3}\left[G\left(\frac{\lambda}{\bar{w}}+\frac{1}{2}\left[1-\frac{G \lambda^{2}}{1+\lambda^{2}}\right]\right)\left(1-\frac{G \lambda^{2}}{1+\lambda^{2}}\right)\left(K_{0}-\frac{1}{1+\lambda^{2}}\right)\right. \\
& -\frac{G^{2} \lambda^{2}}{1+\lambda^{2}}\left(1-\frac{G \lambda^{2}}{1+\lambda^{2}}\right)\left(K_{0}(1)-\frac{1}{1+\lambda^{2}}\right) \\
& +\frac{1}{2}\left(\frac{\lambda}{\bar{w}}-1\right)\left(1-\frac{G \lambda^{2}}{1+\lambda^{2}}\right)^{2}+\frac{1}{2}\left(1-\frac{G \lambda^{2}}{1+\lambda^{2}}\right)^{3} \\
& +G^{2}\left(\frac{\lambda}{\overline{\mathrm{w}}}+1\right) \int \bar{u}_{z_{v s}} \alpha(\operatorname{vol}) \\
& +G^{2}\left(\frac{\lambda}{\bar{w}}-1\right) \int\left[\bar{u}_{\Psi_{\text {Vs }}}^{2}+\bar{u}_{r_{\text {Vs }}^{2}}^{2}\right] d(\text { vol }) \\
& +G^{2}\left(1-\frac{G \lambda^{2}}{1+\lambda^{2}}\right) \int\left[\bar{u}_{z_{v s_{R}}^{2}}+\bar{u}_{\Psi}^{2}\right] d(\text { vol }) \\
& -2 \frac{G^{3} \lambda^{2}}{1+\lambda^{2}} \int \bar{u}_{z_{\text {vs }}} \bar{u}_{z_{\text {vs }}} d(\operatorname{vol})
\end{aligned}
$$

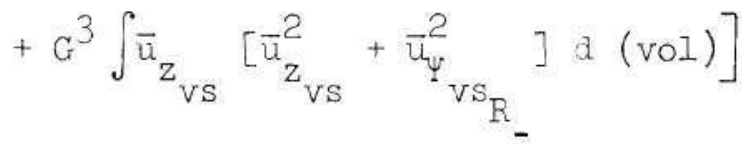


of the remaining integral terms the first three are evaluated in the calculation of $\epsilon_{0}$ for the thrust coefficient. The last two must be numerically integrated for the $\overline{\mathrm{w}}=0$ case in a similar manner.

From equation (47) the power can be written as

$$
P=\infty=T V_{\infty}+E
$$

so that defining a power coefficient as

$$
C_{P}=P /\left[\rho(\Omega R)^{3} \pi R^{2}\right]
$$

yields

$$
C_{P}=(\lambda-\bar{w}) C_{T}+e
$$

The power requirement for a constant diameter wake can also be calculated directly by the Kutta-Joukowski theorem. An increment of torque is given by $d Q=b p V_{\text {axial }} \Gamma r d r$ where $V_{\text {axial }}$ is given by

$$
\begin{aligned}
V_{\text {axial }} & =V_{\infty}+u_{\xi} \sin \varphi+u_{\zeta} \cos \varphi \\
& =V_{\infty}+w\left(1-\frac{G \lambda^{2}}{x^{2}+\lambda^{2}}\right) .
\end{aligned}
$$

Then

$$
d Q=\rho(2 \pi R w \lambda) K(x)\left[V_{\infty}+w\left(1-\frac{G \lambda^{2}}{x^{2}+\lambda^{2}}\right)\right] R^{2} x d x
$$

and

$$
Q=\rho(\pi R)^{2} R w \lambda\left[\left(V_{\infty}+w\right) 2 \int_{0}^{1} K(x) x d x-2 G w \lambda^{2} \int_{0}^{1} K(x) \frac{x}{x^{2}+\lambda^{2}} d x\right]
$$


From equation (41)

$$
k=2 \int_{0}^{1} K(x) x d x
$$

Defining the remainine integral as

$$
\mu=2 \int_{0}^{1} K(x) \frac{x}{x^{2}+\lambda^{2}} d x
$$

the power can be written as

$$
P=Q \Omega=\rho\left(\pi R^{2}\right)(\Omega R)^{3} \bar{w} \lambda^{2}[\hbar-G \lambda \vec{w} \mu] .
$$

Then, since $K=G_{0}$ and $\mu=G_{0}$, the power coefficient is given by

$$
C_{P}=G \bar{w} \lambda^{2}\left[\kappa_{0}-G \bar{w} \lambda \mu_{0}\right] .
$$

\section{Efficiency}

The indueet efficiency is defined as $\eta_{i}=T V_{\infty} / P$. Thus from equation (50)

$$
\eta_{i}=\frac{(\lambda-\overline{\mathrm{w}}) C_{T}}{(\lambda-\overline{\mathrm{w}}) \mathrm{C}_{T}+\epsilon} .
$$

Effieiency may also be written as

$$
\eta_{i}=(\lambda-\bar{w}) C_{T} / C_{P} .
$$


CHAPTER V

\section{NUMERICAL PROCEDURES}

In the preceding chapters the model and its mathematical solution have been outlined in terms of the basic procedures and developments required. To obtain such a solution it is necessary to evaluate the velocity contributions of a filament of unknown strength at an arbitrary location in the flow field of that filament. This evaluation will be carried out by numerically calculating the integrals of equations (9), (10) and (11). Using these results a system of linear equations will be developed in terms of the unknown filament strengths of the system by equating the sums of velocities at control points in the ultimate wake to the required normal velocities at these points. In order to specify the parameters of these equations, decisions will be made about the manner of subdividing the vortex sheets into finite strength filaments and the placement of control points on the sheets.

After the system of equations is determined and solved, the integrals of equations (45) and (46) will be evaluated in order to calculate the thrust and power of the ducted fan. In addition the values of $K_{0}(x), k_{0}$ and $\mu_{0}$ will be evaluated.

\section{Evaluation of Velocity Components}

The evaluation of the velocity components associated with a single finite strength helical vortex filament at an arbitrary location in the flow field of the filament depends (in this analysis) on a 
numerical integration of an integrand which is solely a function of the geometry of the filament and the point at which the velocity is to be calculated. The expressions for the components, equations (9), (10), and (11), are repeatcd here for convenience.

$$
\begin{aligned}
& \frac{\Delta u_{r}}{w}=\frac{Y}{4 \pi R w} \int_{-\infty}^{\infty}\left\{\vec{r}^{\prime} \tan \varphi_{R} \sin \left(\Psi^{\prime}-\Psi\right)\right. \\
& \left.+\bar{r}^{\prime}\left(\bar{z}-\bar{z}_{0}^{\prime}-\Psi^{\prime} \tan \varphi_{R}\right) \cos \left(\Psi^{\prime}-\Psi\right)\right\} \frac{d \Psi^{\prime}}{\bar{p}^{3}} \\
& \frac{\Delta u_{\xi}}{w}=\frac{\gamma \cos \varphi}{4 \pi R w} \int_{-\infty}^{\infty}\left\{\bar{r}^{\prime} \tan D_{R}\left(\frac{\bar{r}}{\bar{r}}+\frac{\bar{r}^{\prime}}{\bar{r}}-2 \cos \left(\Psi^{\prime}-\Psi\right)\right)\right. \\
& \left.+\left(\bar{z}-\bar{z}_{0}^{\prime}-\Psi^{\prime} \tan D_{R}\right) \sin \left(\Psi^{\prime}-\Psi\right)\right\} \frac{a \Psi^{\prime}}{\bar{p}^{3}} \\
& \frac{\Delta u_{\zeta}}{w}=\frac{y \cos \varphi}{4 \pi R w} \int_{-\infty}^{\infty}\left\{\bar{r}^{\prime 2}-\bar{r} \bar{r}^{\prime} \cos \left(\Psi^{\prime}-\Psi\right)\right. \\
& -\tan ^{2} \omega_{R}\left(1-\frac{\bar{r}^{\prime}}{\bar{r}} \cos \left(\Psi^{\prime}-\Psi\right)\right) \\
& \left.-\frac{\vec{r}^{\prime}}{\bar{r}} \sin \left(\Psi^{\prime}-\Psi\right) \tan \oplus_{R}\left(\bar{z}-\bar{z}_{\circ}^{\prime}-\Psi^{\prime} \tan \emptyset_{R}\right)\right\} \frac{d \Psi^{\prime}}{\bar{p}^{3}}
\end{aligned}
$$

where

$$
\begin{aligned}
& \overline{\mathrm{P}}^{2}=\bar{r}^{2}+\bar{r}^{\prime 2}-2 \bar{r} \bar{r}^{\prime} \cos \left(\Psi^{\prime}-\Psi\right)+\left[\bar{z}-\bar{z}_{0}^{\prime}-\Psi^{\prime} \tan \varphi_{\mathrm{R}}\right]^{2}, \\
& \varphi=\tan ^{-1}\left(\frac{1}{\bar{r}} \tan \varphi_{R}\right),
\end{aligned}
$$


and the primed dimensions $\bar{r}^{\prime}, \bar{z}_{0}^{\prime}$, $\psi^{\prime}$ refer to the location of an elemental length of the vortex filament. The unprimed dimensions refer to the location of the point at which the velocity is to be calculated. The computations needed to numerically evaluate these integrals may be simplified by converting their limits from $-\infty \leq \psi^{\prime} \leq \infty$ to $0 \leq \psi^{\prime} \leq \infty$. This is done in the usual manner by splitting the integral. at $\Psi^{\prime}=0$, switching the limits on the $-\infty \leq \psi^{\prime} \leq 0$ portion and redefining $\psi^{\prime}$ to $-\psi^{\prime}$ in the negative range. The resulting velocity component relations are (using the substitution $\lambda=\tan \varphi_{R}$ )

$$
\begin{aligned}
& \frac{\Delta u_{r}}{\mathrm{w}}=\frac{\gamma}{4 \pi R w} \int_{0}^{\infty}\{[ \bar{r}^{\prime} \lambda \sin \left(\Psi^{\prime}-\Psi\right) \\
&\left.+\bar{r}^{\prime}\left[\bar{z}-\bar{z}_{0}^{\prime}-\lambda \Psi^{\prime}\right] \cos \left(\Psi^{\prime}-\Psi\right)\right] \frac{1}{\overline{\mathrm{P}}_{1}^{3}} \\
&-\left[\bar{r}^{\prime} \lambda \sin \left(\Psi^{\prime}+\Psi\right)\right. \\
&\left.\left.-\bar{r}^{\prime}\left[\bar{z}-\bar{z}_{0}^{\prime}+\lambda \Psi^{\prime}\right] \cos \left(\Psi^{\prime}+\Psi\right)\right] \frac{1}{\overline{\mathrm{P}}_{2}^{3}}\right\} d \Psi \\
& \frac{\Delta u_{\xi}}{\mathrm{w}}=\frac{\gamma \cos \varphi}{4 \pi R w} \int_{0}^{\infty}\left\{\overline { r } ^ { \prime } \left[\lambda \left(\frac{\bar{r}^{\prime}}{\bar{r}}+\frac{\bar{r}}{\bar{r}^{\prime}}-2 \cos \left(\Psi^{\prime}-\Psi\right)\right.\right.\right. \\
& \\
&\left.\quad+\left[\bar{z}-\bar{z}^{\prime}-\lambda \Psi^{\prime}\right] \sin \left(\Psi^{\prime}-\Psi\right)\right] \frac{1}{\overline{\mathrm{P}}_{\perp}^{3}}
\end{aligned}
$$




$$
\begin{aligned}
& +\bar{r}^{\prime}\left[\lambda \left(\frac{\bar{r}^{\prime}}{\bar{r}}+\frac{\bar{r}}{\bar{r}}-2 \cos \left(\Psi^{\prime}+\Psi\right)\right.\right. \\
& \left.\left.-\left[\bar{z}-\bar{z}_{0}^{\prime}+\lambda \Psi^{\prime}\right] \sin \left(\Psi^{\prime}-\Psi\right)\right] \frac{1}{\bar{p}_{2}^{3}}\right\} d \Psi^{\prime}, \\
& \frac{\Delta u_{\zeta}}{w}=\frac{\gamma \cos \varphi}{4 \pi R w} \int_{0}^{\infty}\left\{\left[\bar{r}^{\prime 2}-2 \bar{r} \bar{r}^{\prime} \cos \left(\Psi^{\prime}-\Psi\right)\right.\right. \\
& -\lambda^{2}\left[1-\frac{\bar{r}^{\prime}}{\bar{r}} \cos \left(\Psi^{\prime}-\Psi\right)\right] \\
& \left.-\frac{\bar{r}^{\prime}}{\bar{r}} \lambda \sin \left(\psi^{\prime}-\Psi\right)\left[\bar{z}-\bar{z}_{c}^{\prime}-\lambda \Psi^{\prime}\right]\right] \frac{1}{\bar{p}_{1}^{3}} \\
& +\left[\vec{r}^{\prime 2}-\overrightarrow{r r}^{\prime} \cos \left(\Psi^{\prime}+\Psi\right)\right. \\
& \left.-\lambda^{2}[]-\frac{\bar{r}^{\prime}}{\bar{r}} \cos \left(\Psi^{\prime}+\Psi\right)\right] \\
& \left.\left.+\frac{\bar{r}^{\prime}}{\overline{r^{\prime}}} \lambda \sin \left(\Psi^{\prime}+\Psi\right)\left[\bar{z}-\bar{z}_{0}^{\prime}+\lambda \Psi^{\prime}\right]\right] \frac{1}{\overline{\mathrm{p}}_{2}^{3}}\right\} d \Psi^{\prime},
\end{aligned}
$$

where

$$
\begin{aligned}
& \overline{\mathrm{P}}_{I}^{2}=\bar{r}^{2}+\bar{r}^{\prime 2}-2 \bar{r}^{\prime} \cos \left(\psi^{\prime}-\psi\right)+\left[\bar{z}-\bar{z}_{0}^{\prime}-\lambda \Psi^{\prime}\right]^{2} \\
& \overline{\mathrm{P}}_{2}^{2}=\bar{r}^{2}+\bar{r}^{\prime 2}-2 \bar{r}^{\prime} \cos \left(\Psi^{\prime}+\Psi\right)+\left[\bar{z}-\bar{z}_{0}^{\prime}+\lambda \Psi^{\prime}\right]^{2} .
\end{aligned}
$$


For convenience in the ensuing discussion the integrands of the velocity components are defined by $f_{r}, f_{\xi}$ and $f_{\zeta}$ such that

$$
\begin{aligned}
& \frac{\Delta u_{r}}{w}=\frac{\gamma}{4 \pi R w} \int_{0}^{\infty} f_{r}\left(\Psi^{\prime} ; \bar{r}, \bar{r}^{\prime}, \bar{z}, \bar{z}_{0}^{\prime}, \Psi, \lambda\right) d \Psi^{\prime} \\
& \frac{\Delta u_{\xi}}{w}=\frac{\gamma \cos \varphi}{4 \pi R w} \int_{0}^{\infty} f_{\xi}\left(\Psi^{\prime} ; \vec{r}, \bar{r}^{\prime}, \bar{z}, \bar{z}_{0}^{\prime}, \Psi, \lambda\right) d \Psi^{\prime} \\
& \frac{\Delta u_{\zeta}}{w}=\frac{\gamma \cos \varphi}{4 \pi R w} \int_{0}^{\infty} f_{\zeta}\left(\Psi^{\prime} ; \bar{r}, \bar{r}^{\prime}, \bar{z}, \bar{z}_{0}^{\prime}, \Psi, \lambda\right) d \Psi^{\prime}
\end{aligned}
$$

The numerical integrations proceed in a straightforward manner; $\frac{\Delta u_{r}}{w}$ will be used as the illustrative example. The integration is performed by subdividing each turn of the helix $\left(\Delta \Psi^{\prime}=2 \pi\right)$ into $\mathrm{k}$ subintervals of included angle $2 \pi / k$. $f_{r}$ is calculated at $\psi^{\prime}=0,2 \pi / k, 2(2 \pi / k), \ldots$ $\mathrm{k}(2 \pi / \mathrm{k})$ and these values are stored as they are calculated. Then the contributions of these segments of the helical filament are summed by a trapezoidal rule integration such that

$$
\begin{aligned}
\delta_{I}\left(\frac{\Delta u_{r}}{w}\right)=(2 \pi / k)\left[f_{r}(0)+\frac{1}{2}\left(f_{r}(0)+f_{r}(2 \pi / k)\right)\right. \\
+f_{r}(2 \pi / k)+\frac{1}{2}\left(f_{r}(2 \pi / k)+f_{r}\left(2 \frac{2 \pi}{k}\right)\right)+ \\
\\
\ldots+f_{r}\left([k-1] \frac{2 \pi}{k}\right) \\
\left.+\frac{1}{2}\left(f_{r}\left([k-1] \frac{2 \pi}{k}\right)+f_{r}\left(k \frac{2 \pi}{k}\right)\right)\right] .
\end{aligned}
$$


(Note that $\delta_{I}$ is a coefficient of velocity subject to multiplication by the non-dimensional filament strength $\gamma / 4 \pi \mathrm{Rw}.) \delta_{1}$ measures the contribution of the first turn of the helical filament (and its reflection for $\left.-2 \pi \leq \Psi^{\prime} \leq 0\right)$ to the radial velocity associated with the filament. This result is stored and the contribution of the turn defined by $2 \pi \leq \psi^{\prime} \leq 4 \pi$ (and its reflection) is calculated in the same manner yielding $\delta_{2}\left(\frac{\Delta u_{r}}{w}\right)$. Then $\delta_{2}$ is compared to the sum of $\delta_{1}$ and $\delta_{2}$. A percent change is defined as $100 \times\left|\delta_{2} /\left(\delta_{1}+\delta_{2}\right)\right|$. If this change is not sufficiently small then $\delta_{3}$ is calculated and the comparison is made again according to $100 \times\left|\frac{\delta_{l}}{l}\right|$. The process is continued until $\sum_{i} \delta_{i}$

the percent change in the coefficient of $\frac{\Delta u_{r}}{w}$ is less than some specified tolerance. The coefficient is then set equal to the sum of the contributions of all terms for which $\delta_{i}$ was calculated.

Clearly, the accuracy of this result depends on the choice for $k$ and the specified tolerance. The magnitudes of these parameters depend in general on the geonetrical values $\vec{r}, \bar{r}^{\prime}, o_{R}$ and on the number of blades in the fan. It would be possible to control these values very closely by a process of repeated calculations and comparisons within a given integration. For example, $\delta_{I}$ could be evaluated with $k=90$, then reevaluated with $k=120$, ggain wi-h $k=150,180$ and so on until two successive values were identical to an arbitrarily specified number of significart digits. Then $\delta_{2}$ would bo handied in the same manner. Similarly the $\delta_{i}$ contributions would be summed until the final contribution failed to change the sum in the chosen number 
of significant digits. It is apparent that such a procedure could be enormously time consuming, and the choice for the number of significant digits to be carried must be made very conservatively. Choosing the best alternative at hand, reliance is made on the specification of minimum requirements over a broad range of cases.

The values of $k$ and the tolerances for the velocity components were sized somewhat subjectively on the basis of their effects on solutions of the wake vorticity distribution. The method of sizing a parameter consists of fixing all but one of these at very conservative levels and systematically varying the remaining parameter over a wide range. This procedure is repeated for various values of $b$ and $\lambda$ and the resulting wake vortex strength distributions are compared. For example, $k$ was varied over a range from 90 to 720 . It was found that for $k$ greater than 180 the solutions for wake vorticity were essentially identical for $b$ and $\lambda$ taken over very wide ranges. That is, the value of $K_{0}(I)$ at $b$ and $\lambda$ was changed by less than $1 / 2$ of one percent by increasing $k$ from 180 to 720 . On the basis of this result $k$ was fixed at 180 for all subsequent solution, obviating the need for any repetitive calculations.

The permissable tolerances for summing the $\delta_{i}$ contributions to $\frac{\Delta u_{\xi}}{w}$ and $\frac{\Delta u_{c}}{w}$ were treated in a similar manner. The minimum values were found to vary with the choice of $b$ and $\lambda$ but in general could be fairly large due to a favorable compensation of the tmuncation errors. For example, the value for $\frac{\Delta u_{C}}{w}$ for ten turns of a filament might be only 95 percent of the value for 30 turns, but the errors for the $\frac{\Delta u_{C}}{w}$ values for the other filament,s would be nearly the same so that solutions for 
wake vorticity (or for $K(1)$ ) would differ by perhaps $1 / 4$ of one percent. Thus it was decided that the tolerances on $\frac{\Delta u_{\xi}}{w}$ and $\frac{\Delta u_{c}}{w}$ could be set at two percent tor all subsequent solutions without incurring significant errors.

For the calculation of $\frac{\Delta u_{r}}{w}$ the truncation tolerance was found to be strongly depentunt on the characteristic axial separation of two adjacent inner helical sheets as measured at the wake boundary. This characteristic length may be non-dimensionalized by the wake circumference to yield

$$
\tilde{z}_{C}=\frac{2 \pi R \lambda / b}{2 \pi R}=\lambda / b
$$

When $\tilde{z}_{c}$ is small problems arise in accurately calculating the radial velocities. That is, when a calculation point lies on the cylindrical. boundary, the radial velocity due to a filament in a helical sheet lying to one side of the point must be summed with the velocity contribution of a filament at the same radial station of the helical. sheet lying to the other side of the point. The radial velocity contributions of two such filaments will be nearly equal in magnitude but large and opposite in sign. Thus an acceptable calculation of the sum of these contributions requires accuracy to a large number of significant aigits.

The primary influence of these calculations on the wake vorticity solution occurs in the sizing of the non-uniform boundary sheet strength. If the percent change of $\left|\delta_{l} / \sum_{I}^{\ell} \delta_{i}\right|$ is not hela sufficiently small the strength distribution of this sheet may become quite irregular, changing sign and generally degencrating. Fortunately, the magnitude of the 
non-uniform boundary sheet strength becomes very small (as compared to $K(x)$ ) when $\tilde{z}_{c}$ becomes small so that the effect of inaccuracies in this sheet strength is of secondary importance to the solution for $K(x)$ and to the calculation of velocity profiles in the wake. (When $\tilde{z}_{c}$ becomes zero the model reduces to the infinite blade case for which the radial velocities vanish along with the strength of the non-uniform boundary sheet.) It was found that the solutions could be adequately controlled if. for $\tilde{z}_{c}<1 / 4$ the tolerance was held to 0.1 percent; for $1 / 4<\widetilde{z}_{c}$ $<1 / 2$ the tolerance was $1 / 2$ percent; and for $1 / 2<\tilde{z}_{c}<1$ the tolerance was held to 1.5 percent. Higher values of $\tilde{z}_{c}$ are not considered to be of practical interest.

\section{System of Linear Equations}

As may be seen in the preceding section, the velocity component contribution of a single filament at any point in its flow field is a linear function of the vortex strength of the filament. If the contribution of $n$ filaments to a velocity component, say $\Delta u_{5} / w$, at a given point are summed then the result is of the form

$$
g_{1} \bar{\gamma}_{1}+g_{2} \bar{\gamma}_{2}+g_{3} \bar{\gamma}_{3}+\ldots .+g_{n} \bar{\gamma}_{n}=\bar{u}_{\mathrm{s}} / \cos \varphi
$$

where $g_{i}$ is the integrated functions of geometry, $f_{\zeta}$, related to the set of filaments (one for each blade) of strength $\bar{\gamma}_{i}$ and to the calculation point, $\bar{r}, \Psi, \bar{z}$. If the filament strengths $\left(\bar{\gamma}_{i}\right)$ are unknown and the geometry of the filaments is known, then specifying a value for $\bar{u}_{\zeta}$ at the calculation point yields a linear equation in $\bar{\gamma}_{1}, \bar{\gamma}_{2} \cdot \cdot \bar{\gamma}_{n}$. If $n$ such calculation points are chosen and the 
velocity component is specified at each point, the result is a system of $\mathrm{n}$ linear equations in $\mathrm{n}$ unknowns. Such a system of equations is used in the solution for the distribution of vorticity in the ultimate wake. The inner helical sheets and the non-uniform boundary sheets must be divided into strips and the strips replaced by finite strength vortex filaments. In order to arrive at some criterion for a minimum number of finite strength vortex filaments to be used to represent a sheet, the blade circulation for an optimum free propeller was calculated using the methods of this chapter for comparison with the results of Goldstein ${ }^{10}$ Solutions were generated using $4,6,8,10,12$, and 18 filaments per sheet. The results, which are considered in greater detail in Appendix III, indicated that ten filaments constituted an adequate approximation to the vortex sheet (i.e., a maximum value of $K(x)$ of about 98 percent of Goldstein's value).

Initial calculations for the ducted fan wake model were performed using ten filaments to replace an inner helical sheet and eight filaments to replace a characteristic portion of the non-uniform boundary sheet. Because of the symmetry of vortex strength in the boundary sheet the eight filaments introduce only four unknowns. Control points were placed between adjacert filaments using ton points on the inner sheet at $\Psi^{\prime}=0$ and three points on the cylinorical boundary. This arrangement is illustrated schematically in Fig. 10. At the ten control points on the inner sheet the contributions to $\bar{u}_{\zeta} / \cos \varphi=1$ are calculated and summed to yield ten equations. At the three control points on the cylindrical boundary the contributions to $\bar{u}_{r}=0$ are summed to yield three equations. A final (14th) equation is written in terms of the 


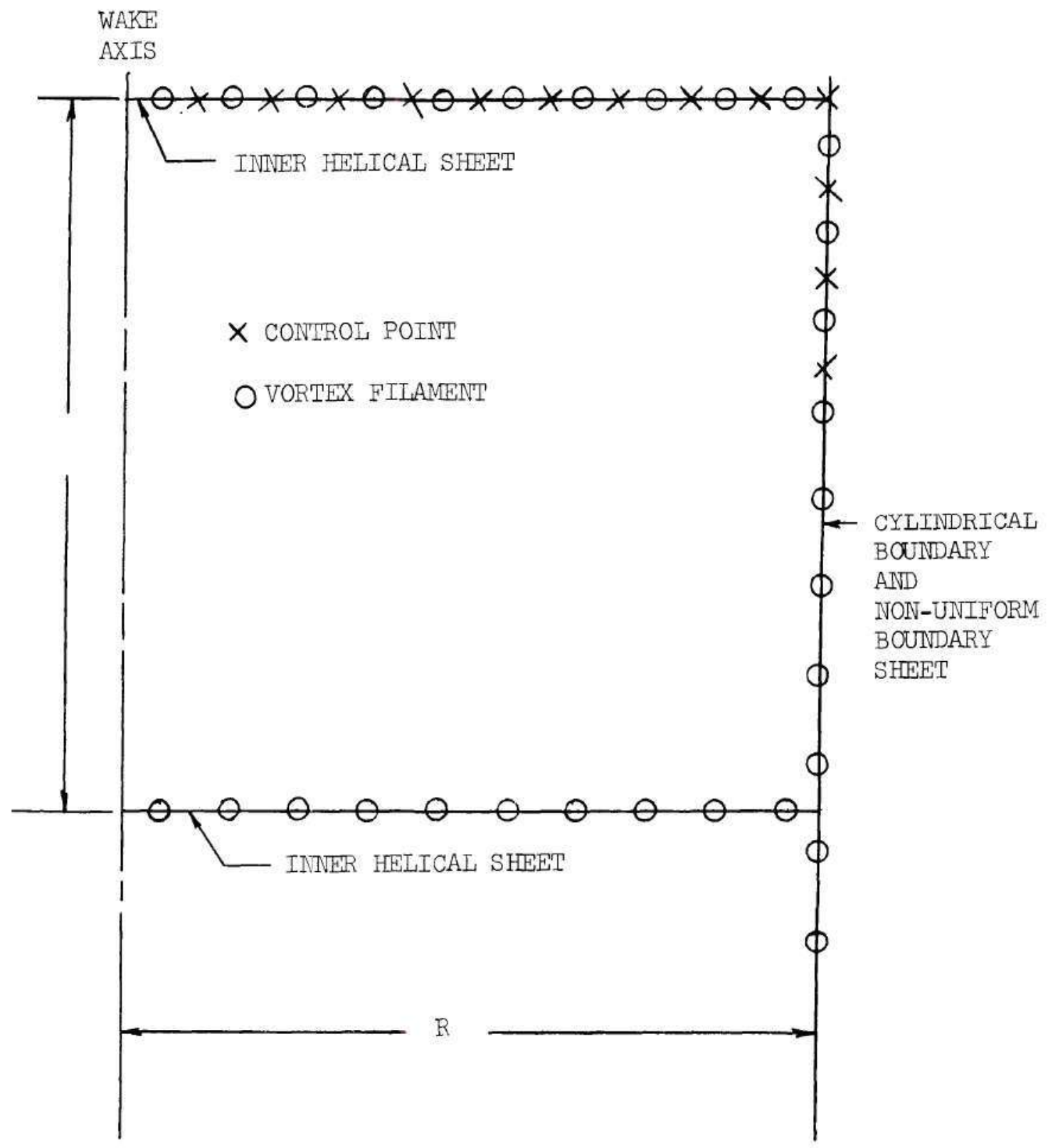

Figure 10. Schematic Diagram of the Arrangement of Vortex Filaments and Control Points in the Ultimate Wake of the Ducted Fan. 
net vorticity. The forms of the right hand elements of the equations are modified to include the effect of the uniform boundary sheet as discussed in Chapter III so that the system of equations takes the form

$$
\begin{aligned}
& A_{1,1} \bar{\gamma}_{1}+A_{1,2} \bar{\gamma}_{2}+\cdots \cdot+A_{1,14} \bar{\gamma}_{14}=\lambda^{2} /\left(1+\lambda^{2}\right) \\
& A_{2,1} \bar{Y}_{1}+A_{2,2} \bar{Y}_{2}+\ldots .+A_{2,14} \bar{Y}_{14}=\lambda^{2} /\left(1+\lambda^{2}\right) \\
& \cdot \\
& \cdot \\
& \text {. } \\
& A_{10,1} \bar{Y}_{1}+A_{10,2} \bar{Y}_{2}+\cdots \cdot+A_{10,14} \bar{Y}_{14}=\lambda^{2} /\left(1+\lambda^{2}\right) \\
& A_{11,1} \bar{Y}_{1}+A_{11,2} \bar{Y}_{2}+\ldots .+A_{11,14} \bar{Y}_{14}=0 \\
& \text {. } \\
& \text {. } \\
& A_{13,1} \bar{v}_{1}+A_{13,2} \bar{\gamma}_{2}+\ldots \cdot+A_{13,14} \bar{v}_{14}=0 \\
& A_{14,1} \bar{Y}_{1}+A_{14,2} \bar{\gamma}_{2}+\ldots .+A_{14,14} \bar{Y}_{14}=-\left(\frac{\lambda}{2 b}\right) /\left(1+\lambda^{2}\right) .
\end{aligned}
$$

$A_{1,1}$ through $A_{10,10}$ are sums of b of the integrated function, $f_{5}$, with the sum taken over the corresponding filaments of each inner helical sheet. $A_{1,11}$ through $A_{10,14}$ are the sums of $b$ integrations of $f_{5}$ for the corresponding filaments of the characteristic portions of the nonuniform boundary sheet. Similarly, $\mathrm{A}_{11,1}$ through $\mathrm{A}_{13,10}$ are integrations of $\mathrm{f}_{2}$ for the inner sheets and $\mathrm{A}_{11,11}$ through $\mathrm{A}_{13,14}$ are the integrated conficients for the non-uniform boundary sheet. $A_{14,1}$ through $A_{14,10}$ are 1.0 and $\mathrm{A}_{14,11}$ through $\mathrm{A}_{14,14}$ are 2.0. $\bar{\gamma}_{1}$ through $\bar{\gamma}_{10}$ are the 
unknown strengths, $\gamma /\left({ }_{4}\right.$ RWG $)$ of the inner sheet filaments and $\bar{\gamma}_{I I}$ through $\bar{\gamma}_{14}$ are the unknown strengths for the non-uniform boundary sheet filaments.

Thorough investigations of the effect of the total numbers of filaments and control point locations and their spacing on the inner sheets indicate that the arrangement shown in Fig. 10 is satisfactory and does not required modification with $\tilde{z}_{c}$ (i.e., $\lambda$ and b). The satisfactory numbers of filaments and control points on the cylindrical boundary were found to be clearly dependent on $\tilde{z}_{c}$. That is, for $\tilde{z}_{c}<1 / 46$ filaments and 2 control points suffice; for $1 / 4<\tilde{z}_{c}<1 / 2$ 8 filaments and 3 control points suffice; and for $1 / 2<\tilde{z}_{c}<1,12$ filaments and 5 control points are adequate. The decisions for adequate arrangements were made rather subjectively on the basis of comparisons of a blade number family of solutions to the exact infinite blade solution by Gray ${ }^{13}$. A further criterion which involves the smoothness of the vorticity listributions on the sheets was used as a neasure of the convergence of the sheet strength distributions.

In Chapter II it was noted that the strength of the non-uniform boundary sheet must be zero at the lines of intersection and must maintain helical. symmetry between lines of intersection. In order to meet these requirements the sheet strength was defined as a series of trigonometric functions such that

$$
\tilde{y}=\sum_{n=1}^{N} a_{n} \sin \left[\frac{2 n-1}{2} \pi \bar{z}\right]
$$


where $N+I$ is the number of control points on the sheet. The series is integrated over a strip width of the sheet to yield the corresponding finite filament strength. If $2 \omega$ represents the strip width such that $\omega=1 / 4 N$ then the filament strength is

$$
\bar{\gamma}=\int_{\bar{z}-\omega}^{\bar{z}+\omega} \bar{\gamma} d \bar{z}=\sum_{n=1}^{N}\left[a_{n}\left(\frac{4 / \pi}{2 n-\lambda}\right) \sin \left[\frac{2 n-1}{2} \pi \omega\right] \cos \left[\frac{2 n-1}{2} \pi \bar{z}\right]\right]
$$

The strength distribution of an inner sheet is also expressed in series form. As $\vec{r}$ approaches 1.0 it is necessary that $\tilde{\gamma}$ become zero so that for points immediately above and below the sheet $\bar{u}_{r}$ may vanish. Since the strength of the sheet is $\tilde{y}=-\frac{d \Gamma(x)}{d x}$ this restriction requires that $\frac{d \Gamma}{d x}$ be zero at $x=1.0$. Thus the bound vortex strength may be written as

$$
\frac{\Gamma(x)}{2 \pi R \omega \lambda}=\sum_{n=1}^{N-1} B_{n} \sin \left[\frac{2 n-1}{2} \pi x\right]
$$

To calculate the strength of a filament of the inner sheet the difference in bound strength is taken at the radial stations corresponding to the edges of the strip which the filament replaces. Thus

$$
\bar{\gamma}=-\sum_{n=1}^{N-1} 2 B_{n} \sin \left[\frac{2 n-1}{2} \pi \omega\right] \cos \left[\frac{2 n-1}{2} \pi x\right] .
$$

The system of linear equations is formed in the same manner as before by suming the velocity component contributions at a given point tue to all of the filaments of unknown strength. However, the unknowns now become the coefficients of the two series for $\bar{\gamma}$. That is, at the point $(\bar{r}, \Psi, \bar{z}) \mathrm{n}$ is fixed and the contributions of all filaments for 
$B_{n}$ (or $a_{n}$ ) are summed to yield the coefficient $A_{i, j}$. For example, set $n=1$ in the $B_{n}$ series so the coefficient for $B_{1}$ is

$$
A_{1,1}=-\sum_{i=1}^{\mathbb{N}} \frac{1}{2 \pi R w} \int_{0}^{\infty} f_{\zeta_{i}} d \Psi^{\prime}\left[\sin \left(\frac{\pi}{2} \omega\right) \cos \left(\frac{\pi}{2} x\right)\right] .
$$

The system formed in this manner is equivalent to the previous system (with discrete filament strengths) with the additional constraints on the end values of the vorticity distributions.

Solution of the linear system for either the filament strengths or the series coefficients is carried out by the Gauss method of successive eliminations as presented by Fadeeva ${ }^{14}$. Having solved for the wake vorticity the blade bound vortex strength distribution may be calculated directly from the series of equation (55). $\kappa_{0}$ may be calculated by integration of the series to yield

$$
\kappa_{0}=\frac{4 b}{\lambda} \sum_{n=1}^{N-1}\left(\frac{2 n-1}{n} \pi\right)^{-1}\left[(-1)^{n+1}-\left(\frac{2 n-1}{\pi}\right)^{-1}\right] B_{n},
$$

and

$$
K_{0}(x)=\frac{2 b}{\lambda} \sum_{n=1}^{N-1} B_{n} \sin \left[\frac{2 n-1}{2} \pi x\right] .
$$

$\mu_{0}$ may be obtained by a simple strip integration based on filament strength such that

$$
\mu_{0}=\frac{4 b}{\lambda}\left\{\sum_{i=1}^{N-1}\left(-\bar{\gamma}_{i}\right) \frac{x_{i}}{\left(x_{i}^{2}+\lambda^{2}\right)^{2}} 2 \omega\right\}
$$


Thrust and Power Integrations

In Chapter IV the thrust and power coefficients were shown to contain integrals of the velocity distribution through a characteristic volume of the wake. These integrals, which must be evaluated numerically are

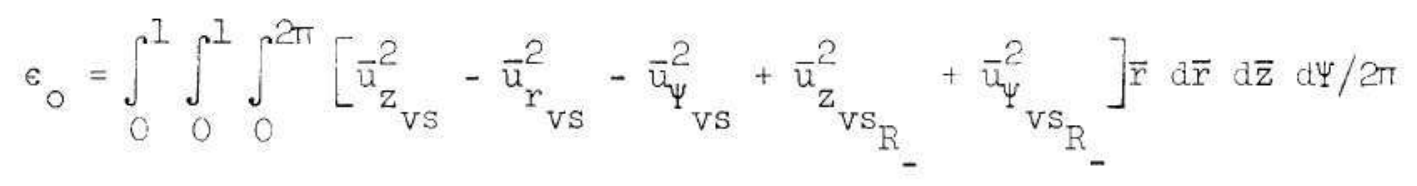

and the integrals occurring in the power calculation

$$
\int_{0}^{1} \int_{0}^{1} \int_{0}^{2 \pi} \bar{u}_{z_{V s}} \bar{u}_{z_{V s}} \bar{r} d \bar{r} d \bar{z} d \Psi / 2 \pi
$$

and

$$
\int_{0}^{1} \int_{0}^{1} \int_{0}^{2 \pi} \bar{u}_{z_{v S}}\left[\bar{u}_{z_{V S_{R}}^{2}}+\vec{u}_{\Psi}^{2}{ }_{v_{S_{R}}}\right] \bar{r} d \bar{r} d \bar{z} d \Psi / 2 \pi
$$

When the strengths of all of the vortex filaments comprising the ultimate wake are known, the components of induced velocity can be calculated at any point in the wake by evaluating the integrals for each of the filaments and multiplying the result by the filament strength. The procedures for integrating and summing the contributions of the filaments are the same as those used in setting up the system of linear equations. These results are then projected to yield the components of induced velocity in the $r-, z-$, and $\psi$ - directions. Due to the helical symetry of the vorticioy and velocity distributions in the ultimate wake, the volume integrations may be performed by 
obtaining a detailed knowledge of the flow field on a $\bar{z} \bar{r}$ - surface. This surface is bounded by the wake axis, the cylindrical boundary, an inner helical sheet, and the radial line midway between two adjacent inner helical sheets. The surface is divided into a network or grid. At the intersection points of the grid the induced velocity components are calculated, their squares are calculated, and all of this information is formed into two-dimensional arrays of data. A typical integration surface is shown schematically in Fig. 11.

Since the motion and vorticity of the wake are known, numerical evaluation of the velocity components at the edges of the $\bar{z} \bar{r}$ - surface is not required. Specifically, at $\bar{r}=0 \quad \bar{u}_{y}=\bar{u}_{r}=0$ and $\bar{u}_{z}=\bar{u}_{\xi_{0}}$. At $\bar{z}=0$, $\bar{u}_{z}=1-G \lambda^{2} /\left(\bar{r}^{2}+\lambda^{2}\right), \bar{u}_{\Psi}=-G \lambda \bar{r} /\left(\bar{r}^{2}+\lambda^{2}\right)$, and $\bar{u}_{r}=-\frac{\lambda}{2 b} \frac{d \Gamma(x)}{d x}$. At $\bar{r}=1 \quad \bar{u}_{r}=0, \bar{u}_{z}=1-G \lambda^{2} /\left(\bar{r}^{2}+\lambda^{2}\right)+2 \tilde{\gamma} \cos \varphi_{R}, \bar{u}_{\psi}=-G \lambda \bar{r} /$ $\left(\bar{r}^{2}+\lambda^{2}\right)+2 \tilde{\gamma} \sin \omega_{R}$. (Here, $\tilde{y}$ is the strength of the non-uniform boundary sheet.) At $\bar{z}=1 / 2 \vec{u}_{r}=0$ but $\bar{u}_{z}$ and $\bar{u}_{\psi}$ must be evaluated numerically. For the arrangement shown in Fig. 1I, all of the velocity components must be evaluated at the internal grid points of which there are 27, and the $\bar{u}_{\psi}$ and $\bar{u}_{z}$ values must be calculated at the 9 lower edge points.

The elements of these two-dimensional arrays of data are then squared to form three new arrays denoted by $\bar{u}_{z_{i, j}}^{2}, \bar{u}_{r_{i, j}}^{2}$ and $\bar{u}_{\Psi}^{2}$. Taking the $\vec{u}_{z_{i, j}}^{2}$ array as an example, the integration may be performed by a simple strip method such that 


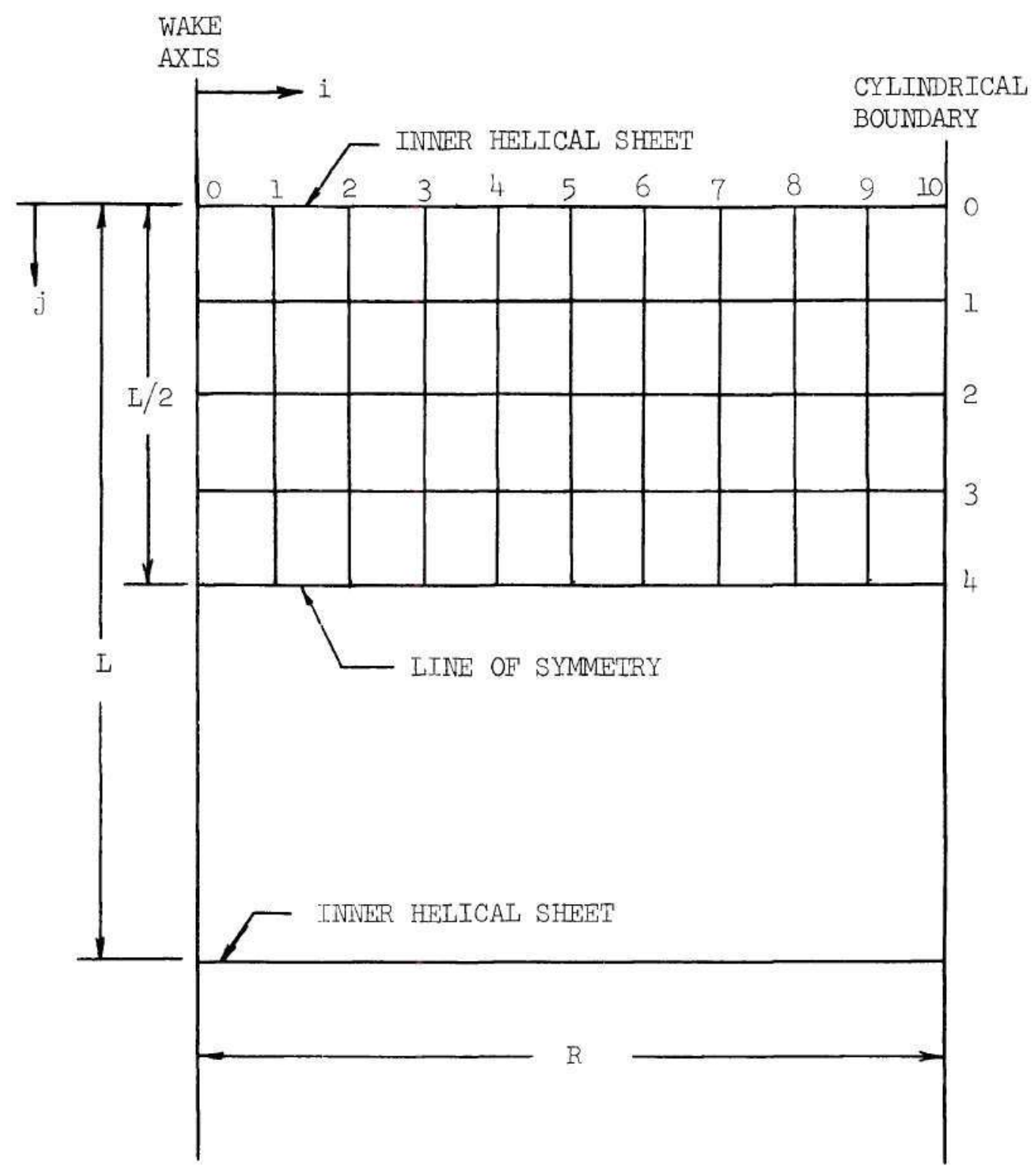

Figure 1I. Network Used in Calculating the Velocity Integrals on the $\bar{z} \bar{r}$ - surface. 


$$
\begin{array}{r}
\int_{0}^{I} \int_{0}^{I} \int_{0}^{2 \pi} \bar{u}_{z} \bar{r} d \bar{r} d \bar{z} d \Psi / 2 \pi=\sum_{i=0}^{I} \sum_{j=0}^{J} \frac{(2 i-1)}{4 I^{2} J}\left[\bar{u}_{z_{i, j}^{2}}+\bar{u}_{z_{i+1, j}}\right. \\
\left.+\bar{u}_{z_{i, j+1}}^{2}+\bar{u}_{z_{i+1, j+1}}^{2}\right]
\end{array}
$$

where $(I+I)$ is the number of grid points in the $\bar{r}$ - direction and $(J+1)$ is the number of grid points in the $\bar{z}-$ direction. For the network illustrated in Fig. 9, $I=10, \mathrm{~J}=4$ and $4 \mathrm{I}^{2} \mathrm{~J}=1600$.

For the integrations involving the (vs) velocities, calculation of the array members is restricted to the filaments associated with the inner helical sheets and the non-uniform boundary sheet. The edge values become: at $\bar{r}=0 \bar{u}_{\Psi_{V S}}=\bar{u}_{r_{V S}}=0, \bar{u}_{z_{V S}}=-1 /\left(1+\lambda^{2}\right)$; at $\bar{z}=0 \bar{u}_{z_{v S}}=\frac{1}{1+\lambda^{2}}-\frac{\lambda^{2}}{\bar{r}^{2}+\lambda^{2}}, \bar{u}_{\Psi_{v S}}=-G \lambda \bar{r} /\left(\bar{r}^{2}+\lambda^{2}\right)$ and $\bar{u}_{r_{v s}}=$ $-\frac{\lambda}{2 b} \frac{\mathrm{d} \Gamma(\mathrm{x})}{\mathrm{dx}} ;$ at $\bar{r}=I \bar{u}_{r_{\text {VS }}}=0, \bar{u}_{\psi_{\text {VS }}}=-\mathrm{C} \lambda /\left(I+\lambda^{2}\right)+2 \tilde{\gamma} \sin \varphi_{\mathrm{R}}$, $\bar{u}_{z_{v S}}=2 \tilde{y} \cos \varphi_{R}$. At $\bar{z}=I / 2 \bar{u}_{r_{v S}}=0$ and $\bar{u}_{z_{v S}}$ and $\bar{u}_{\psi}$ must be evaluated numerically.

The accuracy of the integration technique is clearly dependent on the choice for $I$ and $J$ and on the strip method employed. As a check on the method the $\vec{u}_{z}$ velocity distribution was calculated for several values of $\lambda$ and $b$ and integrated using the method outlined above. From equation (42) of Chapter IV

$$
\int \bar{u}_{z} \mathrm{~d} \operatorname{vol}=\frac{1}{2}\left[\kappa+\bar{u}_{\xi_{0}}\right]
$$


Using values of $I=5$ and $J=4$ the numerical. results agreed with the more exact results of equation (42) to within about 1 percent in all. cases. (See Appendix III.) As a further check the values of $\epsilon_{r}, \varepsilon_{\Psi}$ and $\varepsilon_{z}$ were calculated in the same manner for $\lambda=1.356$ and for increasingly large blade number. Their convergence to the exact value for the infinite blade case ${ }^{13}$ was satisfactorily observed and is considered in more detail in Appendix III. 


\section{CHAPTER VI}

\section{RESULTS}

Following the initial checkouts of the numerical procedures, the methods for the generation and solution of the system of equations for the wake vorticity distribution of the Iightly loaded ducted fan were programmed for the digital computer. The system was solved first for a value of $\lambda=1.356$ with $b=2$ and 4 . The results for the blade bound circulation, $K(x)$, were compared to the electro-potential analogy results of Gray ${ }^{4}$. These comparisons are shown in Fig. 12 and Fig. 13. In both cases the agreement is considered to be good.

Solutions were also generated for comparison to the theoretical results of Tachmindji ${ }^{2}$ who used the velocity potential approach. $K(x)$ is shown for the two methods in Fig. 14 and Fig. 15, with $\lambda=1 / 3$ and $2 / 3$ and $b=4$ in both cases. The agreement is again considered to be good.

For comparison to the exact theory ${ }^{3}$ for a ducted fan having an infinite number of blades, solutions were obtained for $b=2,4,6,8$, 12, 16, and 24 with $\lambda=1.356$. The results for the blade bound vortex strength show excellent convergence to the exact solution as seen in Fig. 16. Since the measure of convergence may be seen more easily in terms of the mass coefficient, the values of $k_{0}$ for increasing blade number are shown in Fig. 17. 


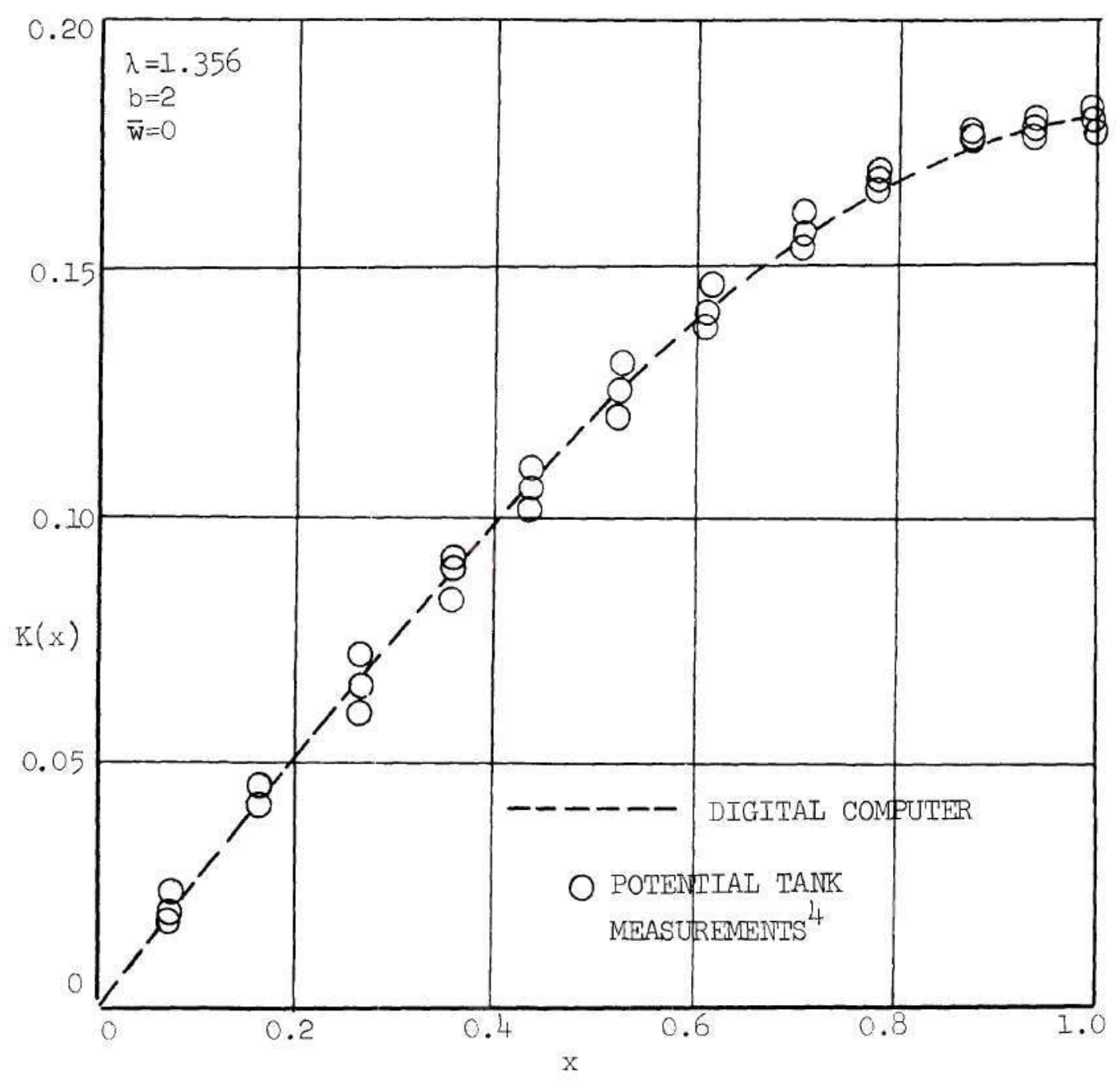

Figure 12. Comparison of Two Methods for Determining the Blade Bound Vorticity for a Iightly Loaded Ducted Fan. 


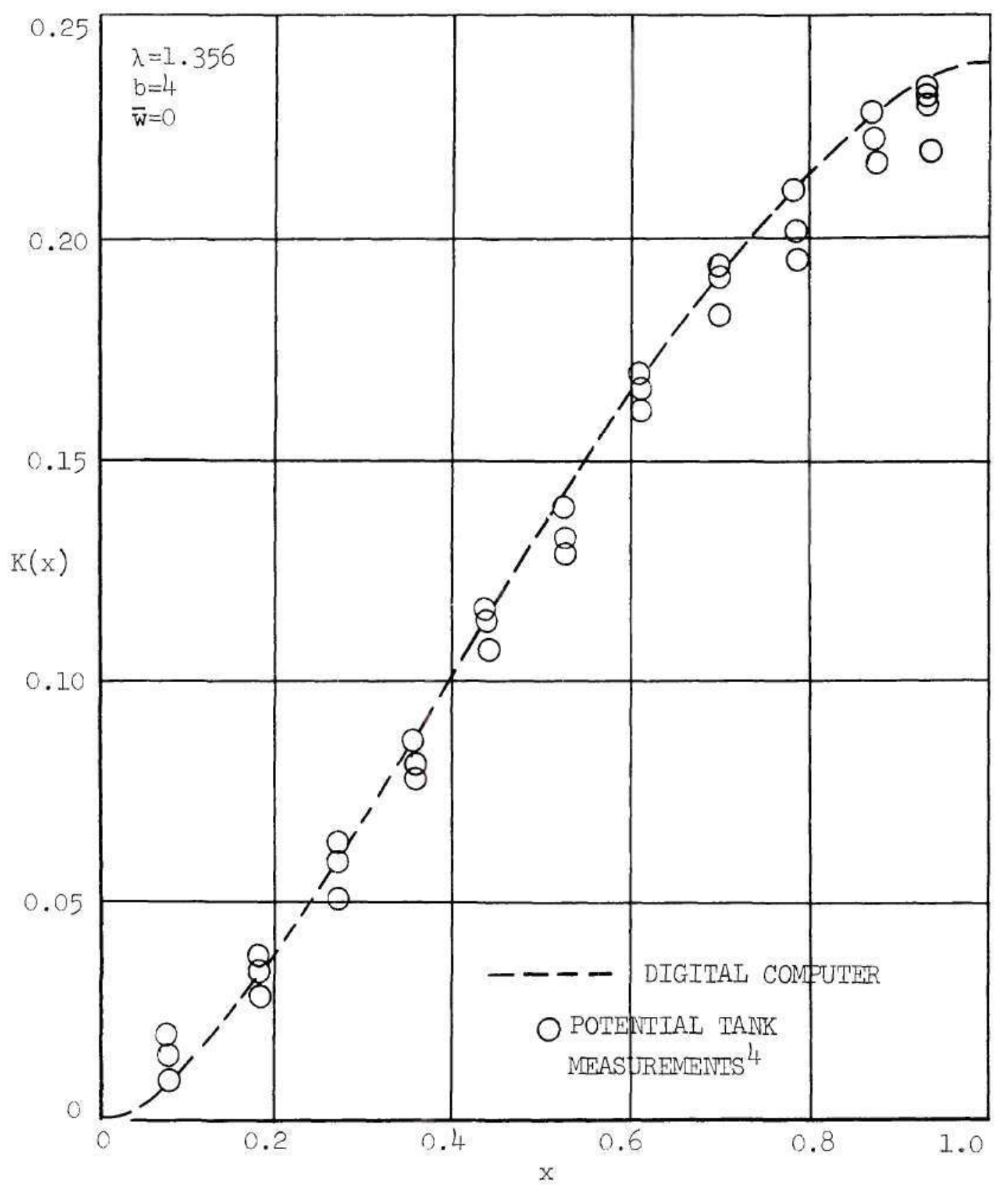

Figure 13. Comparison of Two Methods for Determining the Blade Bound Vorticity for a Lightly Loaded Ducted Fan. 


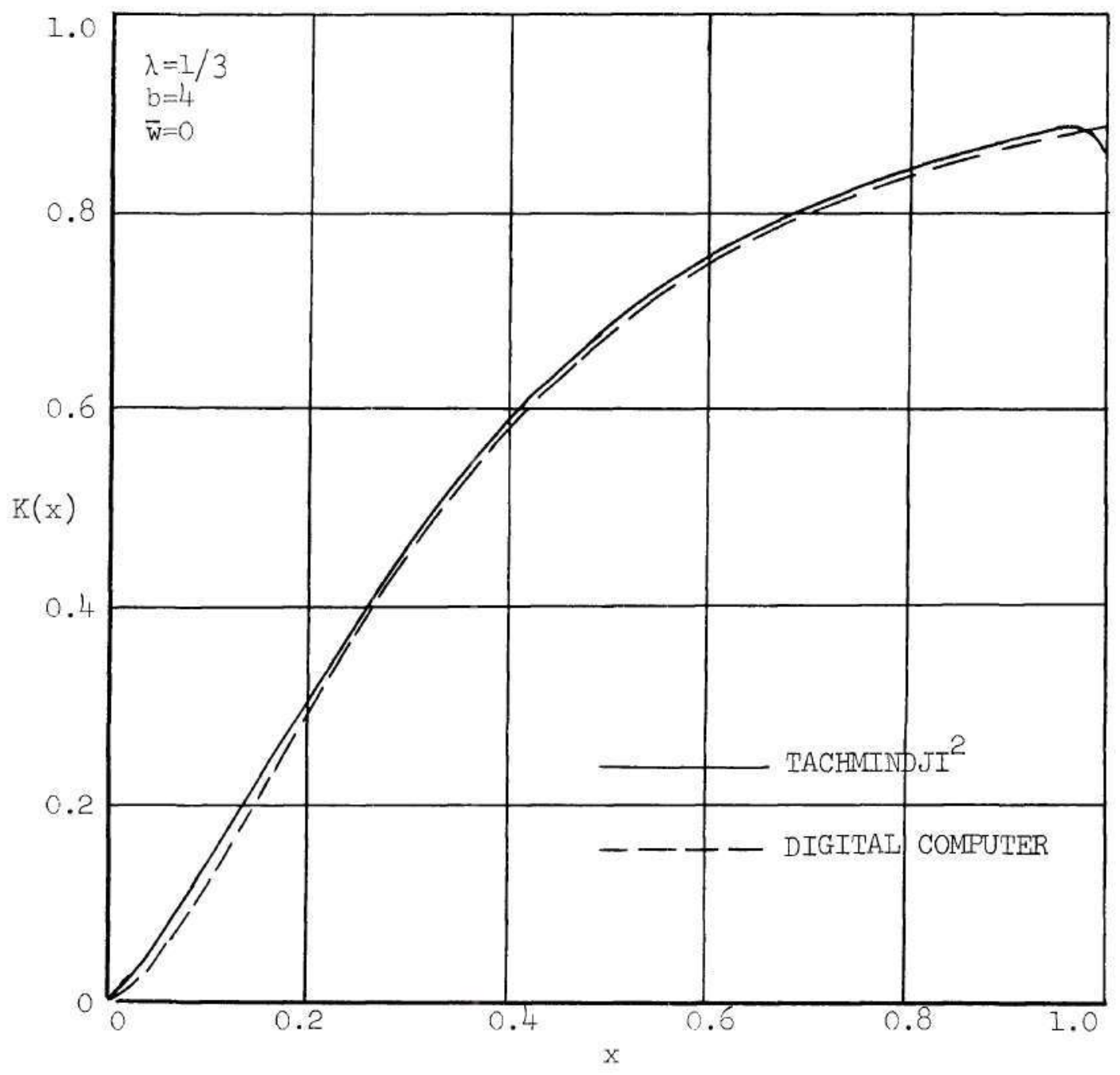

Figure 14. Comparison of Two Methods of Calculating the Blade Bound Vorticity for a Lightly Loaded Ducted Fan. 


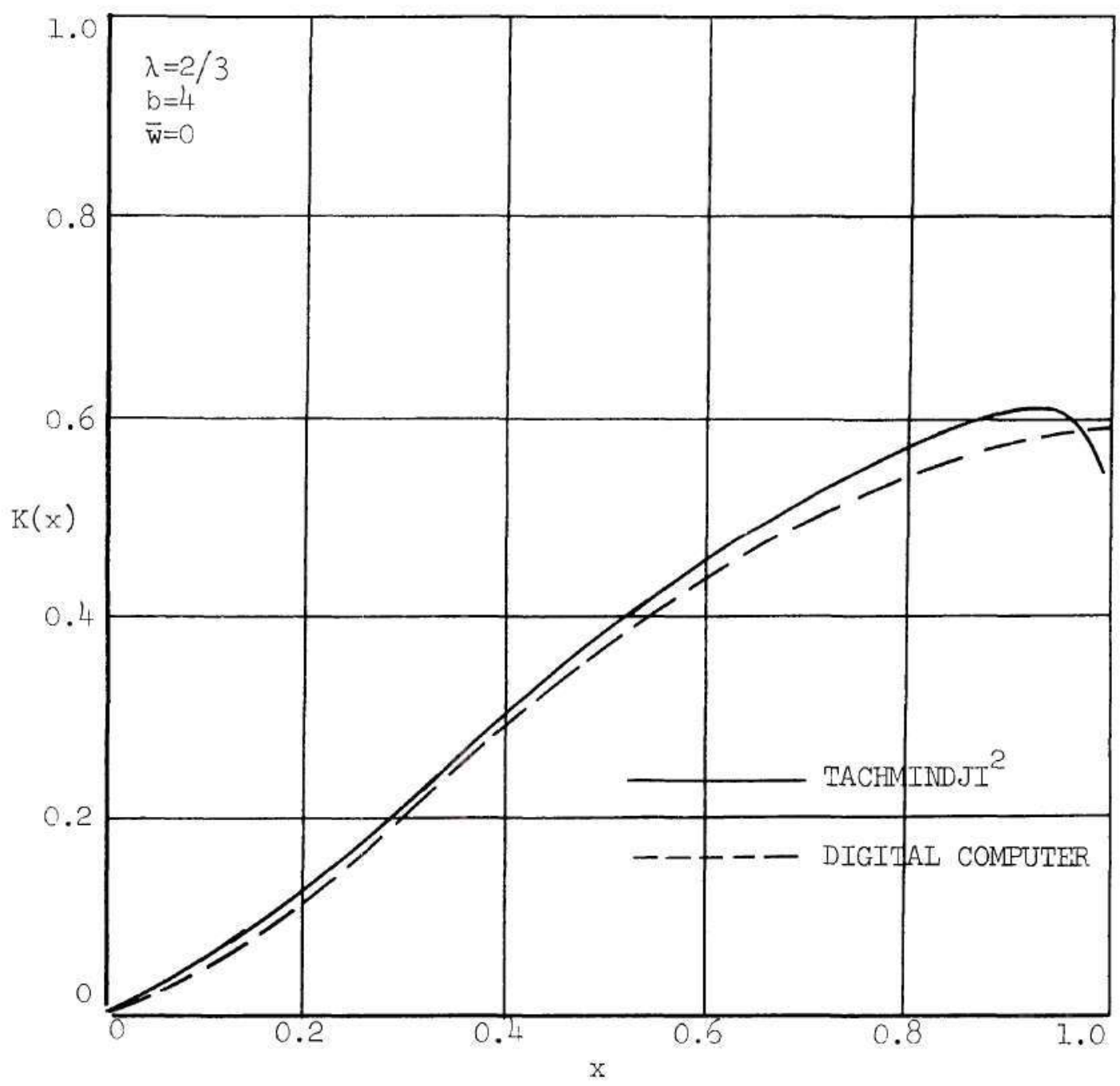

Figure 15. Comparison of Two Methods of Calculating the Blade Bound Vorticity for a Lightly Loaded Ducted Fan. 


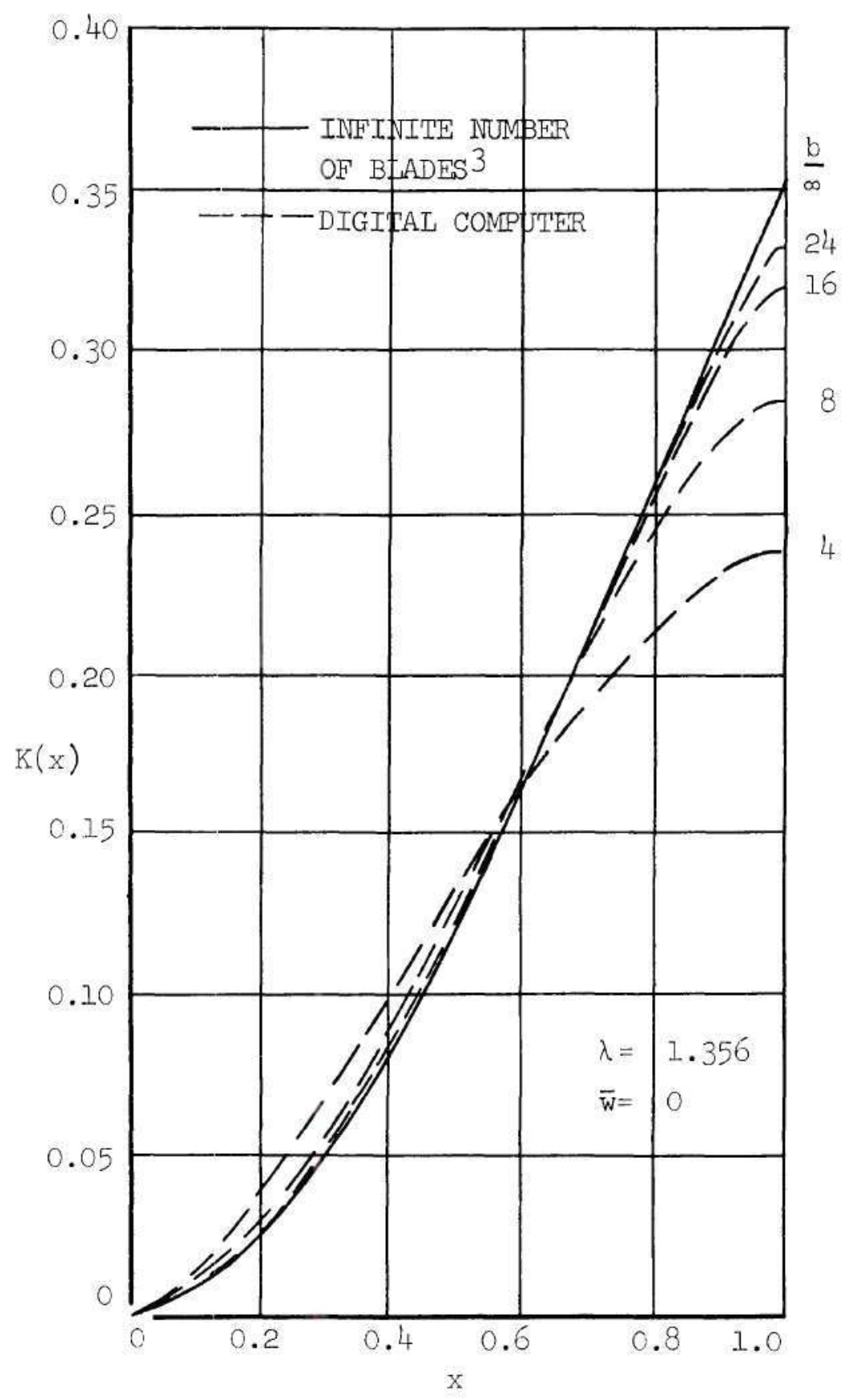

Figure 16. Comparison of the Blade Bound Vorticity for a Lightly Loaded Ducted Fan for Successive Numbers of Blades (Digital Computer) with the Infinite Blade Solution (Exact3). 


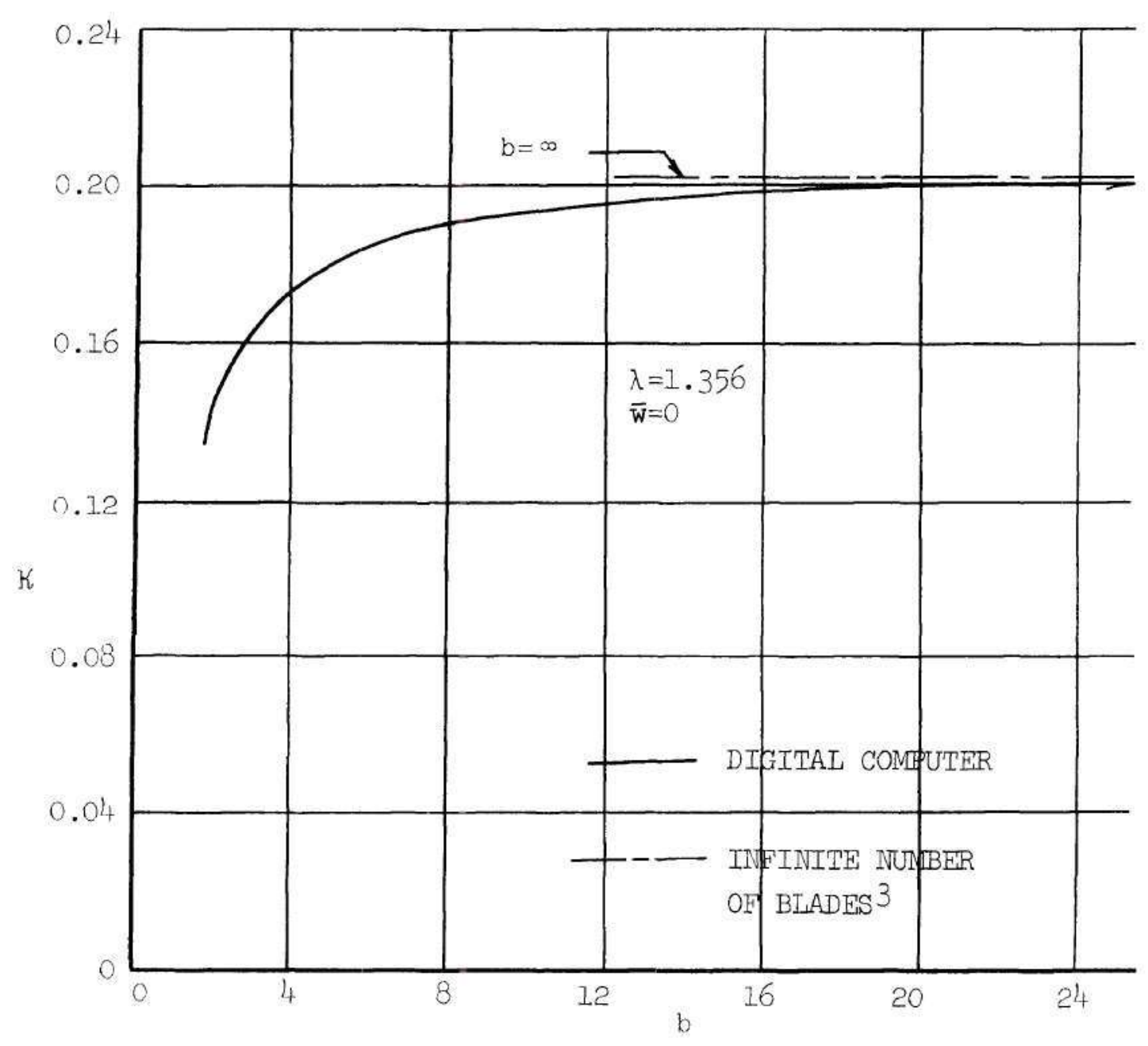

Figure 17. Comparison of Mass Coefficients for Increasing Number of Blades (Digital Computer) with the Mass Coefficient for the Infinite Blade Case (Exact3). 
In order to illustrate the behavior of the design and performance parameters with the the variation of the load parameter, a family of solutions is presented in Figures 18 through 21. Fig. 18 shows the basic lightIy loaded circulation curves, $K_{0}(x)$, for $\lambda=1 / 2$ and $b=2,3,4,6$, and 8 . The variation of the scale factor $G$ with $\bar{w}$ is illustrated in $\mathrm{Fi}$. 19. This result is plotted versus $\bar{w} / \lambda$; since $\overline{\mathrm{w}}$ ranges from zero to $\lambda, \overline{\mathrm{w}} / \lambda$ yields the convenient range of zero to 1.0 with $\overline{\mathrm{w}} / \lambda=1.0$ corresponding to the static thrust condition. The variations of $\mathrm{C}_{\mathrm{T}}$ and $\mathrm{C}_{\mathrm{P}}$ with $\overline{\mathrm{w}} / \lambda$ are shown in $\mathrm{Fig} .20$ and $\mathrm{Fig}$. $2 \mathrm{l}$ for the heavily loaded ducter fans corresponding to the lightly loaded fans of Fig. 18. The exact results for the infinite blade case are included in these figures for comparison and to illustrate the convergence of the results, with increasing blade number, to the exact solution.

In Appendix IV a collection of results is presented in several tables. The range taken for $\lambda$ was $0<\lambda \leq 1$, and for $\bar{w} / \lambda$ from zero to 1.0. For the values of $\lambda$ considered, the blade numbers were taken a.s $2,3,4,6,8,12$ and 16. As may be seen in the tables, the higher blade numbers were not considerci in the calculations for the smaller. values of $\lambda$. Rather, the blade number was increased until the results hat closely approached the exact infinite blale solution. 


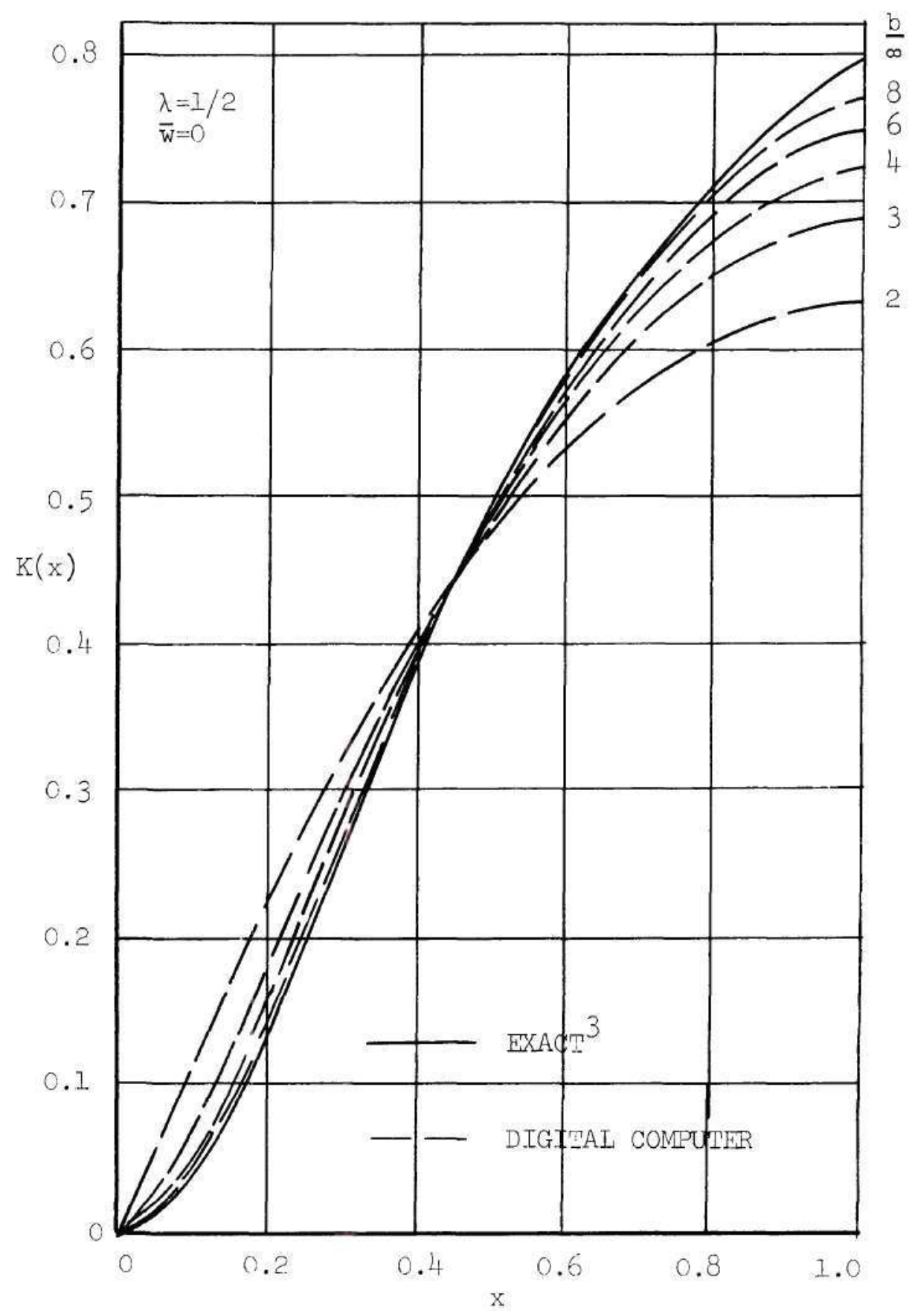

Figure 18. Blade Bound Vorticity for a Family of Lightly Loaded Ducted Fans. 


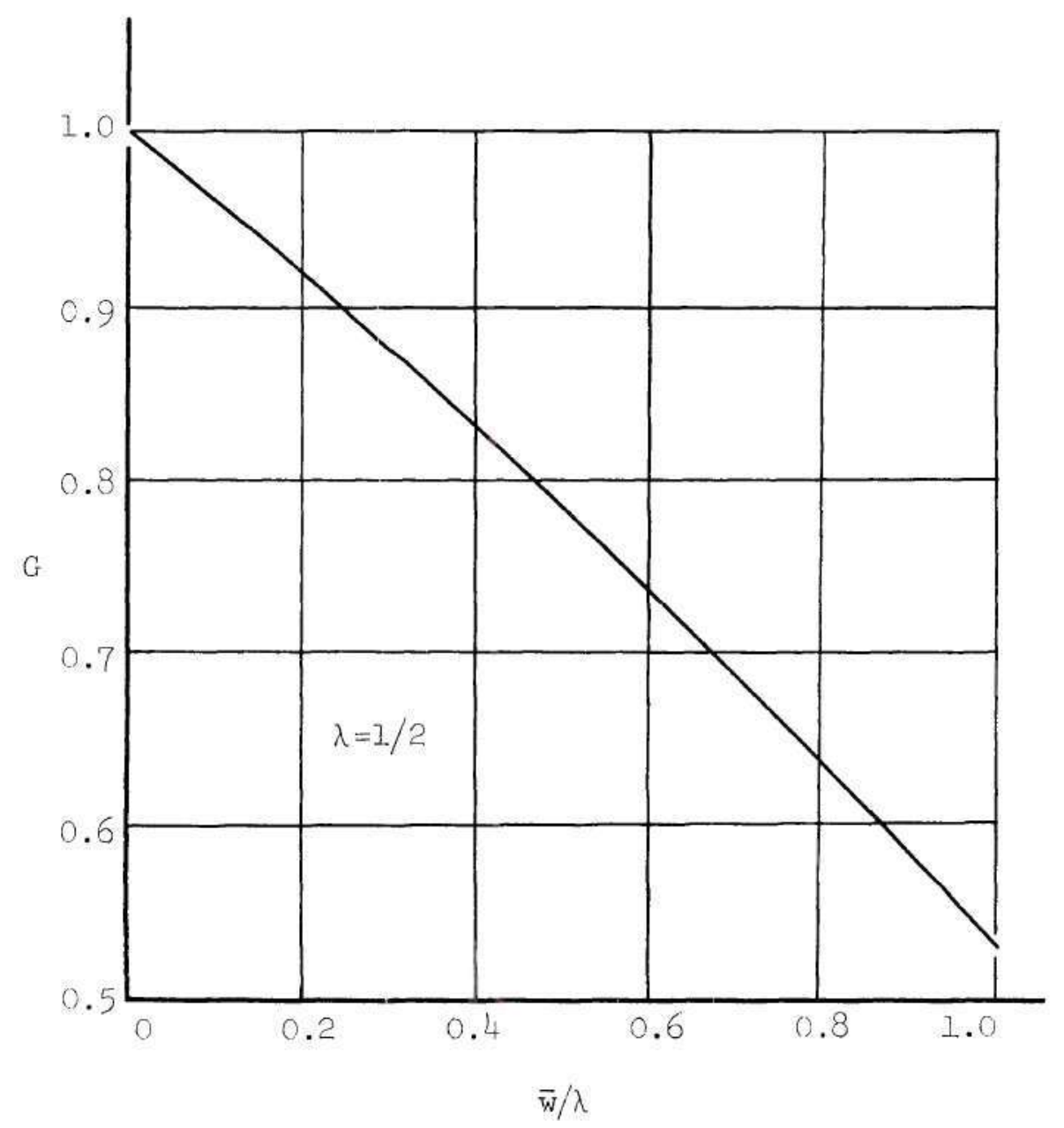

Figure 19. Variation of the Load Scaling Factor. 


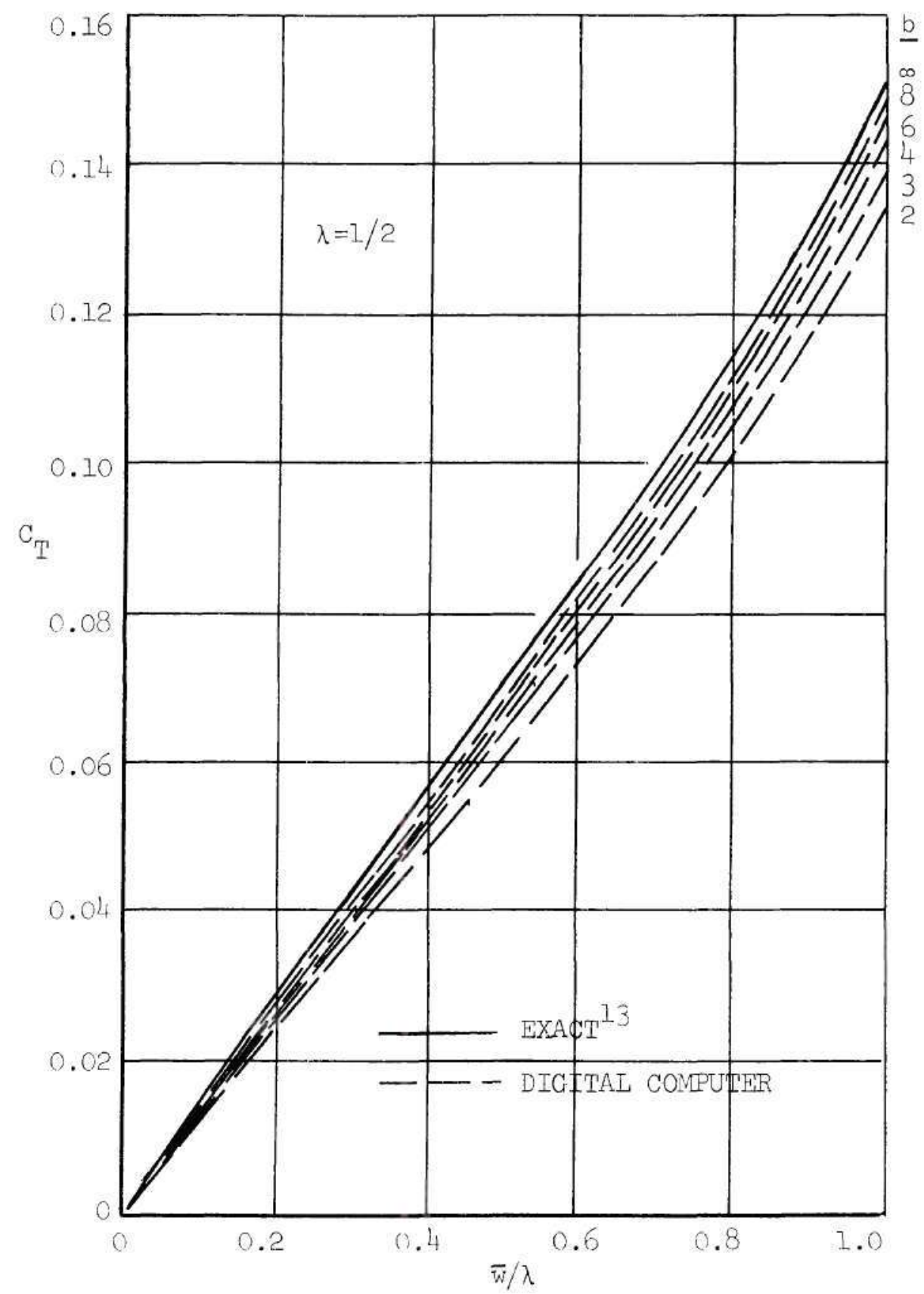

Figure 20. Variation of Thrust Coefficient with Load for the Family of Ducted Fans. 


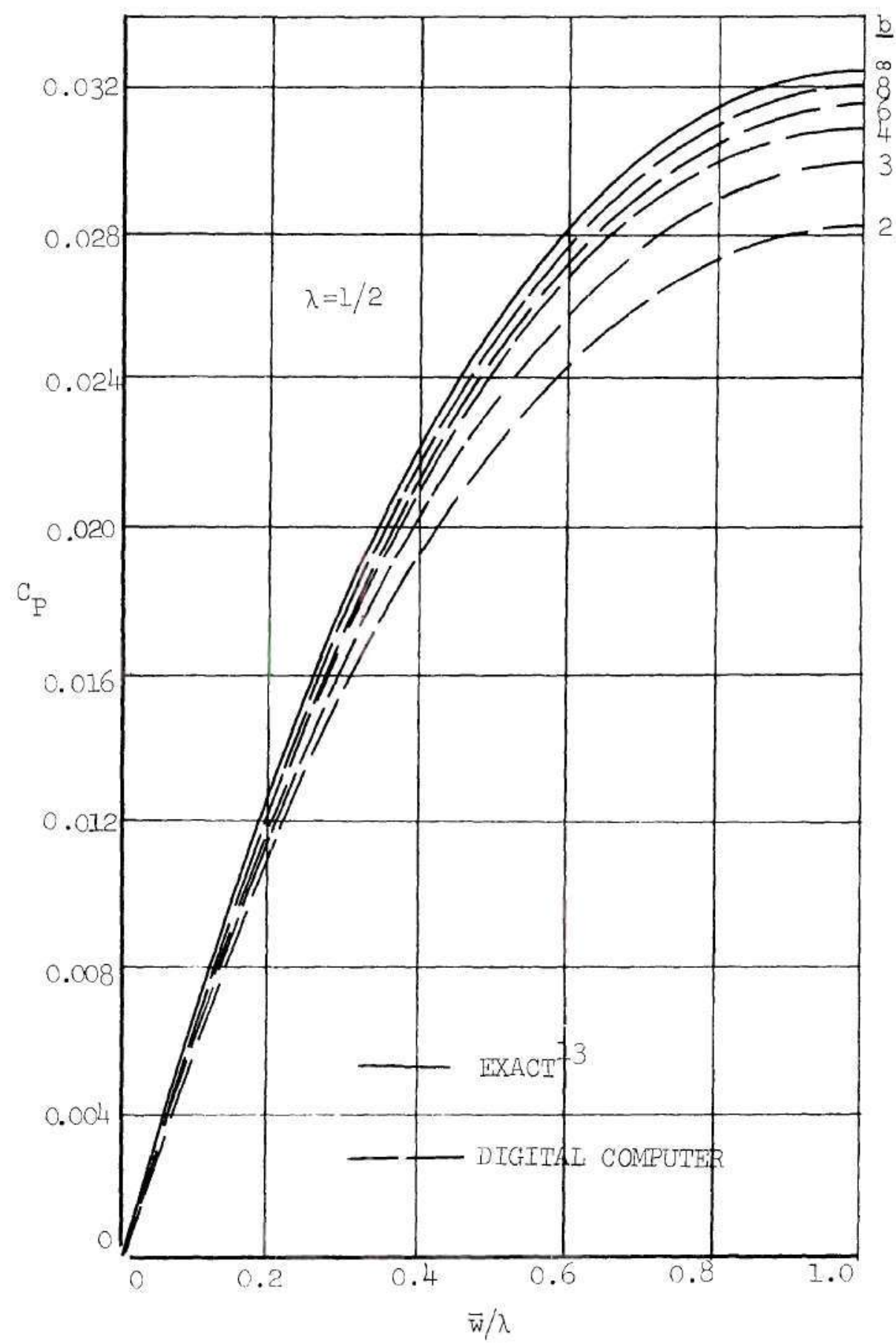

Figure 21. Variation of the Power Coefficient with Ioad for the Family of Ducted Fans. 


\section{APPENDIX I \\ GEOMETRY AND MOTION OF THE INNER HELICAL SHEETS ${ }^{4}$}

The argument as to the geometry and motion of the wake vortex pattern of an optimum ducted single-rotation fan is essentially the same as that presented by Betz ${ }^{9}$ and Theodrosen ${ }^{3}$. Following these approaches, consider a non-optimum ducted fan which is producing the required thrust at the expenditure of the necessary amount of power. At a distance behind the first ducted fan system such that the duct interference velocities are negligible, arrange a second ducted fan having the same number of blades and rotational speed as the first far and so phased with the first fan that each blade intercepts one of the sheet of discontinuity that are shed from the former fan's blade trailing edges. The diameter of the duct of this second system is set equal to the wake diameter so that it intercepts the sheet of discontinuity that is shed from the cirst duct's trailing edge. Assume that the second fan is mounted on an extension of the shaft of the first fan and assume further that neither the second fan nor the duct contribute to the motion of the wake ror disturb the flow in any way. Similarly, place a third ducted fan, and so forth, until a large number of ducted fans are arranged in tandem, all mounted on the shaft of the first, fan, all having the required phase relation, and none contributing to the motion of the wake nor to the thrust or power required. 
In general, certain of the blade elements of the first fan will be operating at relatively high efficiencies while other elements will be operating at relatively low efficiencies. This will be evident in the wake, as will be seen later, by the pitch of the wake spiral with the efficiency being higher where the pitch is lower and vice versa. Suppose now that on the second ducted fan a positive increment of thrust is added to a blade element where the pitch is low and an equal increment of negative thrust is added on the third fan to an element operating in a region where the pitch is high. The thrust of the complete system remains unchanged but the third fan adds more power to the shaft acting as a windmill than the second fan requires to produce the thrust increment so that a net reduction of the power required by the system is realized. (of course skin friction is neglected and it is assumed that the thrust increments are very sma.1. so that the power recovery factor is $100 \%$.) The efficiency of these added increments may be obtained by considering Fig. 22. Using the Kutta-Joukowski theorem, the increment of thrust is

$$
\Delta T=\rho \Delta \Gamma\left(\Omega r-u_{\psi}\right) .
$$

The increment of torque is

$$
\Delta Q=p \Delta \Gamma\left(V_{\infty}+u_{z}\right) r .
$$

This gives for the efficiency

$$
\eta=\frac{V_{\infty} \Delta T}{\Omega \Delta Q}=\frac{\rho \Delta \Gamma\left(\Omega r-u_{\psi}\right) V_{\infty}}{\rho \Delta \Gamma\left(V_{\infty}+u_{z}\right) \Omega r}
$$




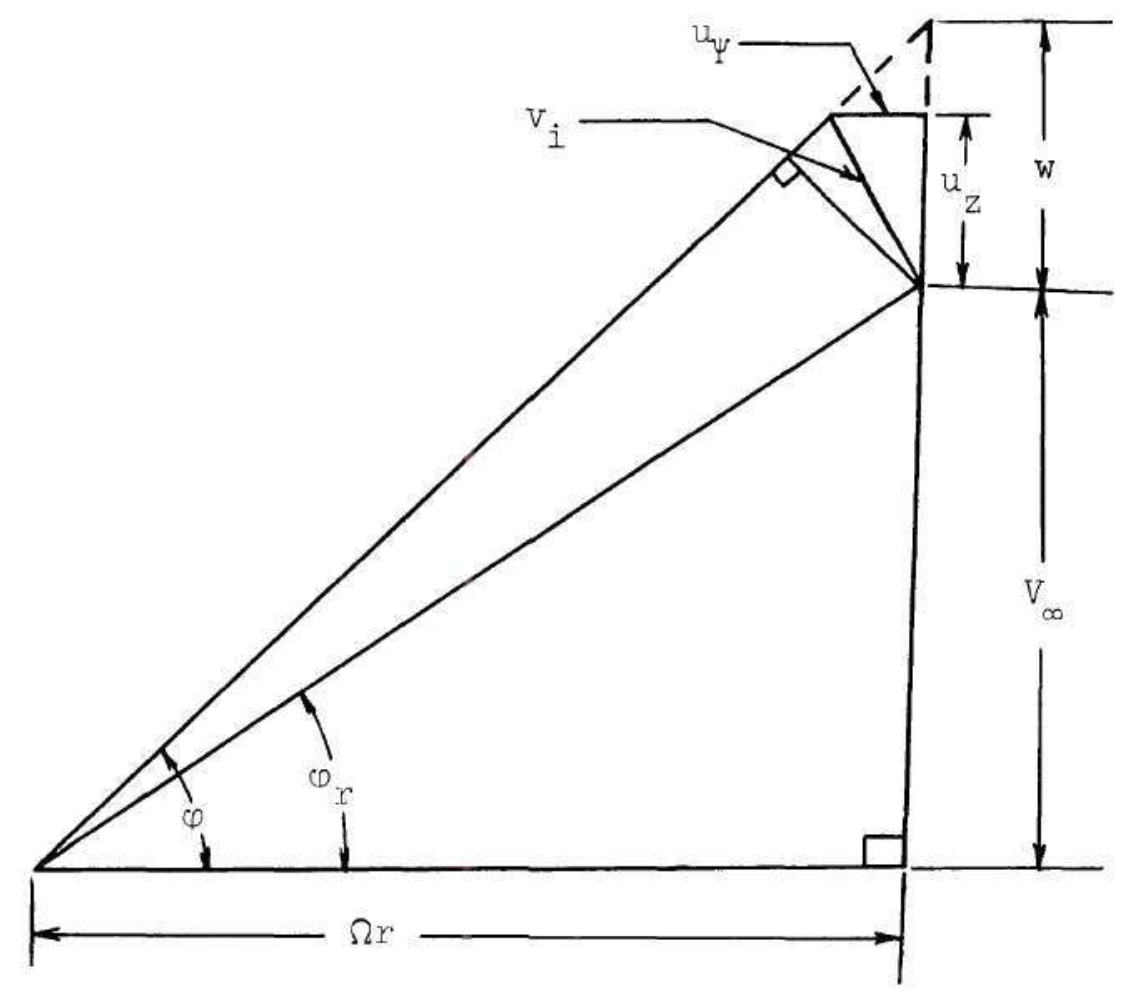

Figure 22. Incremental Thrust Efficiency Diagram. 


$$
\begin{aligned}
& =\frac{V_{\infty}}{V_{\infty}+w}\left(\frac{\Omega r-u_{\psi}}{V_{\infty}+u_{z}}\right) \tan \varphi \\
& =\frac{V_{\infty}}{V_{\infty}+w} \\
\text { or } \quad \eta & =\frac{1}{1+w / V_{\infty}}
\end{aligned}
$$

The elemental efficiency is thus simply a function of the ratio of the apparent velocity of the helical vortex sheet element to the free stream velocity. The process of adding an increment of thmist on a blade element of one fan and removing the same amount on the following fan with a net reduction in power required is continued until no further reduction is realized. At this point the efficiency of the added element of thrust will be the same regardless of the radius at which it is added. From equation (56) this occurs when $\mathrm{w} / \mathrm{V}_{\infty}$ is the same at each blade station for the last fan in the array. The wake behind this last fan represents the wake for the optimum case. Thus the optimum condition is obtained wher the ultimate wake vortex pattern appears to move as a rigid body and the pitch of the inner helical wake spiral is uniform along the radius. The problem, of course, is the determination of the single ducted fan which will yield the same wake configuration as the array. 


\section{APPENDIX II}

VELOCITY FIELD OF THE UNIFORM BOUNDARY SHEET

The uniform boundary sheet of the ultimate wake vortex model consists of equal strength helical vortex filaments of constant and equal pitch wrapped on a right circular cylinder at the pitch angle $\varphi_{\mathrm{B}}$. The strength of all filaments is the same and is given by $\tilde{Y}$, the sheet strength per unit length normal to the filaments.

This sheet can be divided into two sheets to be superposed. One sheet consists of a system of infinitesimal strength ring vortex elements whose axes are the axis of the cylindrical wake boundary surface. The strength of this sheet is given by $\tilde{Y} \cos \varphi_{B}$. The other sheet is a system of straight line filaments of infinitesimal strength lying on the cylinarical boundary surface and parallel to the wake axis. Again, the strength of all filaments is the same and the sheet strength per unit length normal to the filaments is given by $\tilde{Y} \sin \varphi_{B}$. The velocity fields of these two sheets are calculated separately, in terms of a Biot-Savart integration of the filaments of the sheets, and then superposed to yield the velocity field of the entire uniform boundary sheet.

\section{Ring Element Sheet}

From considerations of symmetry, the angular and radial velocities associated with the ring element sheet are identically zero. The geometrical relationships necessary to calculate the axial velocity field of 
this sheet are shown in Fig. 23. From the figure

$$
\begin{aligned}
d y d s^{\prime} & =\tilde{y} \cos \varphi_{B} R d \Psi d z \\
h_{2} & =R-r \cos \Psi \\
h^{2} & =(R-r \cos \Psi)^{2}+z^{2} \\
\overline{\mathrm{P}}^{2} & =R^{2}+r^{2}-2 r R \cos \Psi+z^{2} \\
\cos \beta & =h / \bar{P} \\
\cos \alpha & =h_{2} / h
\end{aligned}
$$

so that

$$
\begin{aligned}
u_{z} & =2 \int_{0}^{\infty} \int_{0}^{2 \pi} \tilde{\gamma} \frac{\cos \varphi_{B}}{4 \pi} \frac{\cos \beta}{\bar{P}^{2}} \cos \alpha R d \Psi d z \\
& =2 \int_{0}^{\infty} \int_{0}^{2 \pi} \tilde{\gamma} \frac{\cos \varphi_{B}}{4 \pi} \frac{(h / \bar{P})\left(h_{2} / h\right)}{\bar{P}^{2}} \\
& =2 \int_{0}^{\infty} \int_{0}^{2 \pi} \tilde{\gamma} \frac{\cos \omega_{B}}{4 \pi} \frac{(1-\bar{r} \cos \Psi) d \Psi d \bar{z}}{\left(1+\bar{r}^{2}-2 \bar{r} \cos \Psi+\bar{z}^{2}\right)^{3 / 2}}
\end{aligned}
$$

where $\bar{x}$ and $\bar{z}$ are nondimensionalized by $R$. Now look at the $\bar{z}-$ integration and define, $a=\left(1+\bar{r}^{2}-2 \bar{r} \cos \Psi\right)$ so that

$$
2 \int_{0}^{\infty} \frac{d \bar{z}}{\left(a+\bar{z}^{2}\right)^{3 / 2}}=\left.\frac{2 \bar{z}}{a\left(a+\bar{z}^{2}\right)^{1 / 2}}\right|_{0} ^{\infty}=\left.\frac{2}{a\left(\frac{a}{\bar{z}^{2}}+1\right)^{1 / 2}}\right|_{0} ^{\infty}=2 / a
$$



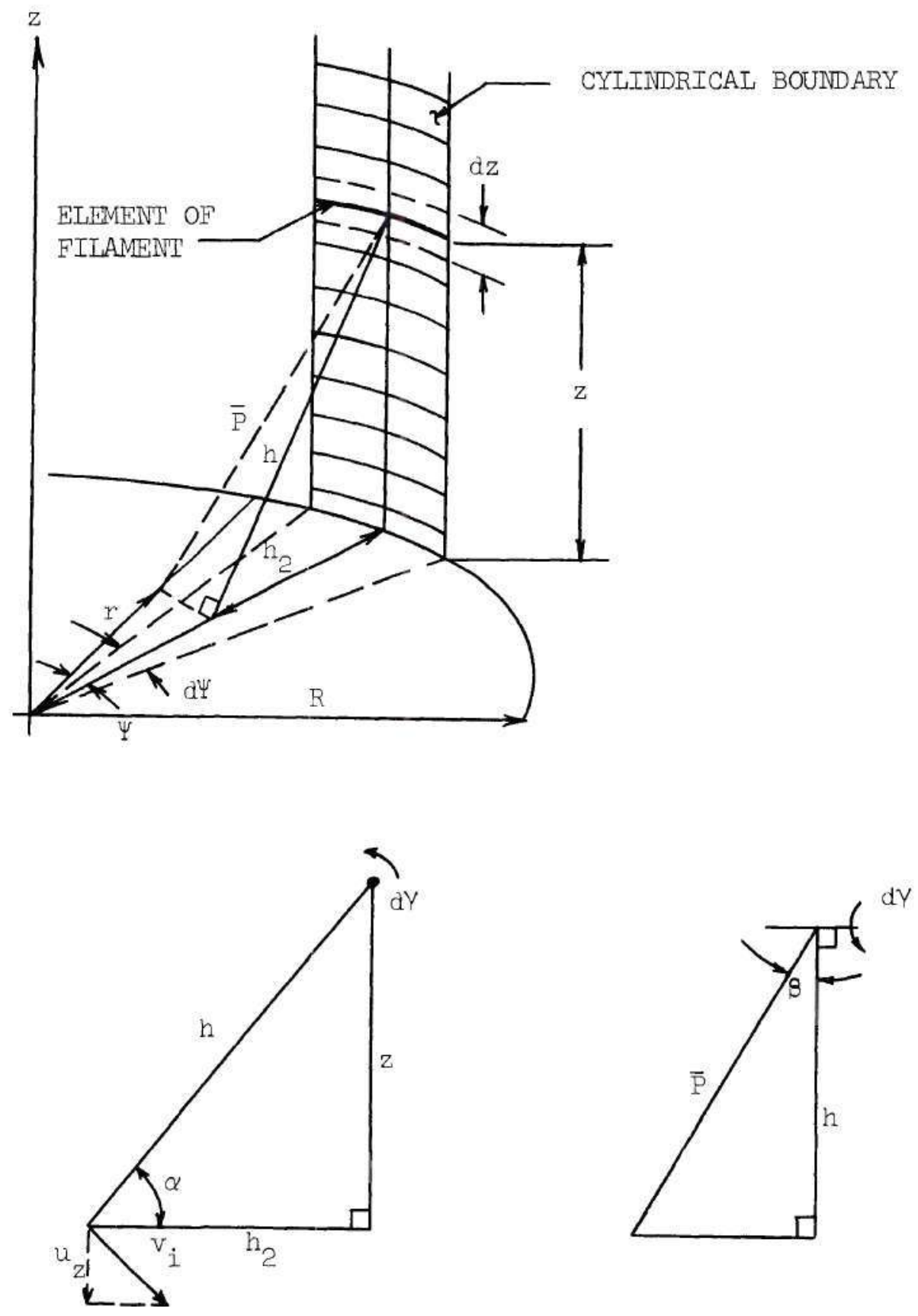

Figure 23. Geometry of the Ring-Element Sheet. 
or

$$
2 \int_{0}^{\infty} \frac{d \bar{z}}{\left(a+\bar{z}^{2}\right)^{3 / 2}}=2 /\left(1+\bar{r}^{2}-2 \bar{r} \cos \psi\right) .
$$

This integral may be found in reference 15 .

Then $u_{z}$ becomes

$$
\begin{aligned}
u_{z} & =\int_{0}^{2 \pi} \tilde{\gamma} \frac{\cos \varphi_{B}}{2 \pi} \frac{(1-\bar{r} \cos \Psi)}{\left(1+\bar{r}^{2}-2 \bar{r} \cos \Psi\right)} d \Psi \\
& =\widetilde{\gamma} \frac{\cos \varphi_{B}}{2 \pi}\left\{\int_{0}^{2 \pi} \frac{d \Psi}{\bar{a}+\bar{c} \cos \Psi}-\bar{r} \int_{0}^{2 \pi} \frac{\cos \Psi d \Psi}{\bar{a}+\bar{b} \cos \Psi}\right\}
\end{aligned}
$$

where $\bar{a}=1+\vec{r}^{2}$ and $\bar{b}=-2 \vec{r}$. But

$$
\frac{\cos \Psi}{\bar{a}+\bar{b} \cos \Psi}=\frac{1}{\bar{b}} \frac{\bar{b} \cos \psi}{\bar{a}+\bar{b} \cos \Psi}=\frac{1}{\overline{\bar{b}}}\left\{1-\frac{\bar{a}}{\overline{\bar{a}}+\bar{b} \cos \Psi}\right\},
$$

so that

$$
u_{z}=\tilde{y} \frac{\cos ^{0} B}{2 \pi}\left\{\int_{0}^{2 \pi}-\frac{\bar{r}}{\bar{b}} d \Psi+\left(I+\frac{\bar{r} a}{\bar{b}}\right) \int_{0}^{2 \pi} \frac{d \Psi}{\bar{a}+\bar{b} \cos \Psi}\right\} .
$$

Now

$$
\int_{0}^{2 \pi} \frac{d \Psi}{\bar{a}+\bar{b} \cos \Psi}=\frac{1}{\bar{a}} \int_{0}^{2 \pi} \frac{d \Psi}{1+\frac{\bar{b}}{\bar{a}} \cos \Psi} \text {, which is of }
$$

the form

$$
\int_{0}^{2 \pi} \frac{d x}{1+a \cos x} \text {, which yields from reference } 15
$$




$$
\int_{0}^{2 \pi} \frac{d x}{1+a \cos x}=\frac{2 \pi}{\left(1-a^{2}\right)^{1 / 2}} \text { for } a^{2}<1 \text { or, } \bar{r}<1 \text { (inside the wake). }
$$

Then

$$
\begin{gathered}
\int_{0}^{2 \pi} \frac{d \Psi}{\bar{a}+\bar{b} \cos \Psi}=\frac{1}{2} \frac{2 \pi}{\left(1-\left(\frac{\bar{b}}{\bar{a}}\right)^{2}\right)^{1 / 2}} \text { and } \\
u_{z}=\tilde{\gamma} \frac{\cos \varphi_{B}}{2 \pi}\left\{2 \pi\left(\frac{-\bar{r}}{-2 \bar{r}}\right)+\left(1+\frac{\bar{r} \bar{a}}{-2 \bar{r}}\right)\left[\frac{1}{1+\bar{r}^{2}}\left(2 \pi /\left(1-\left(\frac{-2 \bar{r}}{1+\bar{r}^{2}}\right)^{2}\right)^{1 / 2}\right)\right]\right\} \\
=\tilde{\gamma} \frac{\cos \varphi_{B}}{2 \pi}\left\{\pi+\frac{1}{2}\left(1-\bar{r}^{2}\right)\left(\frac{2 \pi}{\left(1-\bar{r}^{2}\right)}\right)\right\}=\tilde{\gamma} \frac{\cos \varphi_{B}}{2 \pi}(\pi+\pi)
\end{gathered}
$$

or

$$
u_{z}=\tilde{y} \cos \varphi_{B}
$$

\section{Straight Line Ellement Sheet}

From considerations of symmetry, the axial and radial velocities associated with the straight line element sheet are identically zero. The geometrical relationships necessary to calculate the angular velocity field of this sheet are show in Fig. 24.

The velocity at a point due to the filament can be evaluated directly from reference 16 as

$$
V=\frac{\Gamma}{2 \pi h} \text {, or } V_{i}=\tilde{\gamma} \frac{\sin \varphi_{B} d S}{2 \pi \bar{P}}=\tilde{\gamma} \frac{\sin \varphi_{B} R d \Psi}{2 \pi \bar{P}}
$$




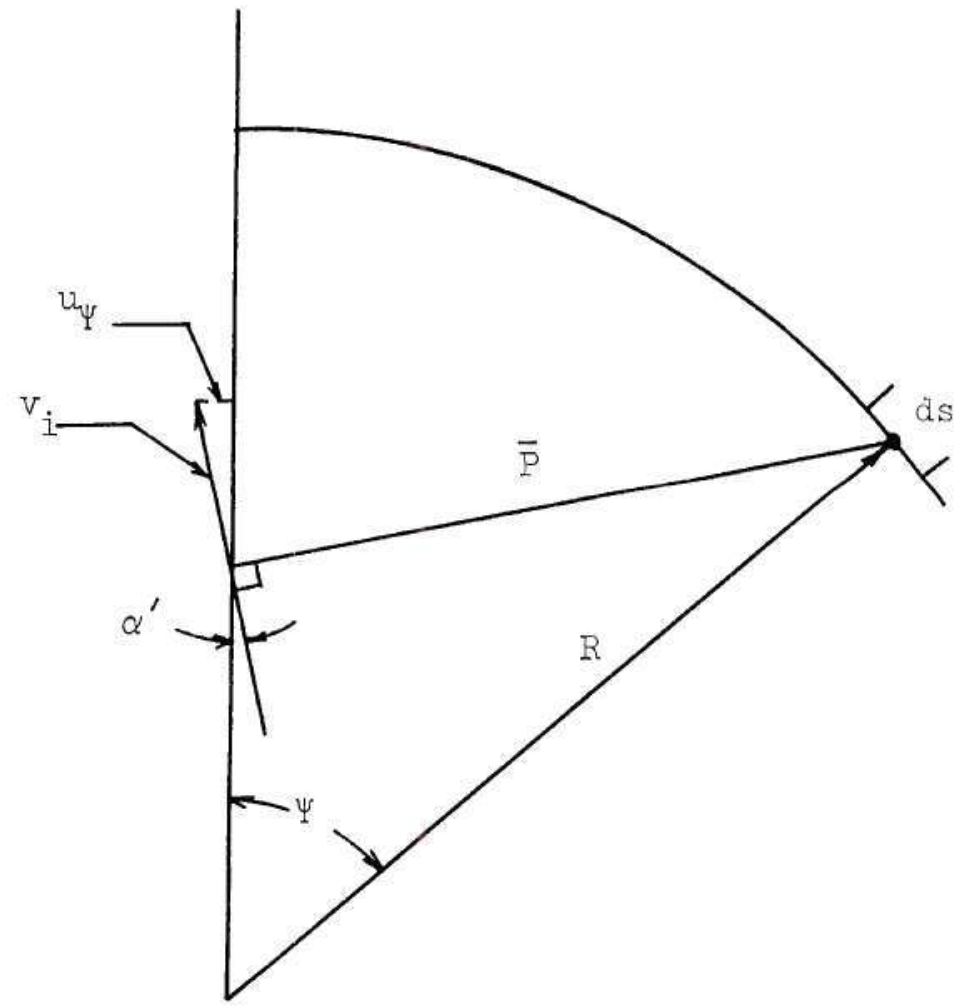

Figure 24. Geometry of the Straight-Line-Element Sheet. 
From the figure $\overline{\mathrm{P}}^{2}=r^{2}+\mathrm{R}^{2}-2 \mathrm{Rr} \cos \Psi$

$$
\sin \alpha=\frac{R^{2}-r^{2}-P^{2}}{2 r \bar{P}} .
$$

Then

$$
d u_{\Psi}=\frac{1}{2 \pi \bar{P}}\left(\frac{r^{2}-R^{2}+\bar{P}^{2}}{2 r \bar{P}}\right) \tilde{\gamma} \sin \omega_{B} R d \Psi
$$

so that

$$
u_{\Psi}=\tilde{\gamma} \frac{\sin \varphi_{B}}{4 \pi}\left\{\frac{2 \pi}{\bar{r}}+\frac{\bar{r}^{2}-1}{\bar{r}} \int_{0}^{2 \pi} \frac{d \Psi}{\bar{a}+\bar{b} \cos \psi}\right\}
$$

where $\bar{a}=1+\bar{r}^{2}$ and $\bar{b}=-2 \bar{r}$. Again, the integral is of the form

$$
\int_{0}^{2 \pi} \frac{d x}{1+a \cos x}=\frac{2 \pi}{\left(1-a^{2}\right)^{1 / 2}}, a^{2}<1 \text {, or } \bar{r}<1
$$

so that

$$
\begin{gathered}
\frac{\bar{r}^{2}-1}{\bar{r}} \int_{0}^{2 \pi} \frac{d \psi}{\left(1+\bar{r}^{2}-2 \bar{r} \cos \psi\right)}=\frac{\bar{r}^{2}-1}{\bar{r}}\left(\frac{1}{1+\bar{r}^{2}}\right)\left(2 \pi /\left(1-\left(\frac{2 \bar{r}}{1+\bar{r}^{2}}\right)^{2}\right)^{1 / 2}\right) \\
=\frac{\bar{r}^{2}-1}{\bar{r}}\left(2 \pi /\left(1-2 \bar{r}^{2}+\bar{r}^{2}\right)^{1 / 2}\right)=\frac{\bar{r}^{2}-1}{\bar{r}}\left(\frac{2 \pi}{1-\bar{r}^{2}}\right)=\frac{-2 \pi}{\bar{r}}
\end{gathered}
$$

Thus

$$
u_{\Psi}=\tilde{\gamma} \frac{\sin \varphi_{B} B}{4 \pi}\left\{\frac{2 \pi}{\bar{r}}-\frac{2 \pi}{\bar{r}}\right\}=0
$$




\section{Superposition}

From the results for the two sheets the velocity field inside the wake due to the uniform boundary sheet is given by

$$
\left.\begin{array}{l}
u_{z}=\tilde{\gamma} \cos \varphi_{B}, \\
u_{r}=0, \\
u_{\Psi}=0 .
\end{array}\right\}
$$




\section{APPENDIX III}

CHECK CASES FOR THE NUMERICAI PROCEDURES

In developing the computer programs to handle the numerical calculations required for the solution of the wake vorticity distribution and the mumerical calculation of thrust and power coefficients, a number of check cases were developed in order to obtain accuracy criteria for the various approximations required for a solution. The first of these checks involves the replacement of helical vortex sheets by a number of finite strength vortices.

The solution for the blade bound vorticity of an optimum free propeller is well-established and has been solved for a number of cases by Goldstein ${ }^{10}$. A numerical model similar to the one employed for the ducted fan was developed in order to check the accuracy of the integration of the velocity contributions of the finite strength helical vortex filaments and to establish a criterion for a minimum number of filaments required to adequately represent a helical vortex sheet. The conditions placed on the solution were as follows. The velocity normal to a helical sheet must be proportional to the local pitch of the sheet along a radial line on the sheet, and the vorticity of a single blade trailing sheet must sum to zero. The blade trailing sheets were divided into J strips, the strips replaced by filaments lying along the centerlines of the strips. Control points at which the normal velocity component was summed and specified were placed 
at $(J-I)$ points midway between the filaments representing the sheet oriented at $\Psi=0$. The arrangement for $J=4$ is shown schematically in Fig. 25. The result is a set of 3 linear equations in 4 unknowns. The 4 th equation is supplied by the zero net vorticity constraint. The system is illustrated as follows.

$$
\begin{aligned}
& A_{1, I} \bar{Y}_{I}+A_{1,2} \bar{Y}_{I}+\ldots .+A_{1, J} \bar{Y}_{J}=\cos \varphi_{r_{1}} \\
& \text {. } \\
& \text {. } \\
& A_{J_{-1,1}} \bar{Y}_{1}+\ldots \cdot \cdot+A_{J-1, J} \bar{Y}_{J}=\cos \varphi_{r_{J-1}} \\
& \bar{\gamma}_{I}+\ldots . . . .+\bar{y}_{J}=0
\end{aligned}
$$

Where, for example, $A_{1,1}$ is the sum of the geometrical integrations of $f_{\zeta}$ for the inboard most filament of the $b$ helical sheets. The system was calculated and solved for $\lambda=1 / 2$ and $b=2$ for values of $J=4,6$, 8, 10, 12, and 18. The results are shown in Fig. 26. Based on these results, it was decided that the ten filament system represents an adequate approximation to the vortex sheets. Greater numbers of filaments improve the solution very slowly and it was felt that the correspondingly larger computation times were not justified.

In order to provide a check on the calculation and integration of the functions of velocity in the ultimate wake, the integral for $\bar{u}_{z}$ was set up in the manner described in Chapter $V$ and evaluated for several cases of $b$ at $\lambda=1 / 2$. The result was compared to the exact value provided by equation (42) according to 


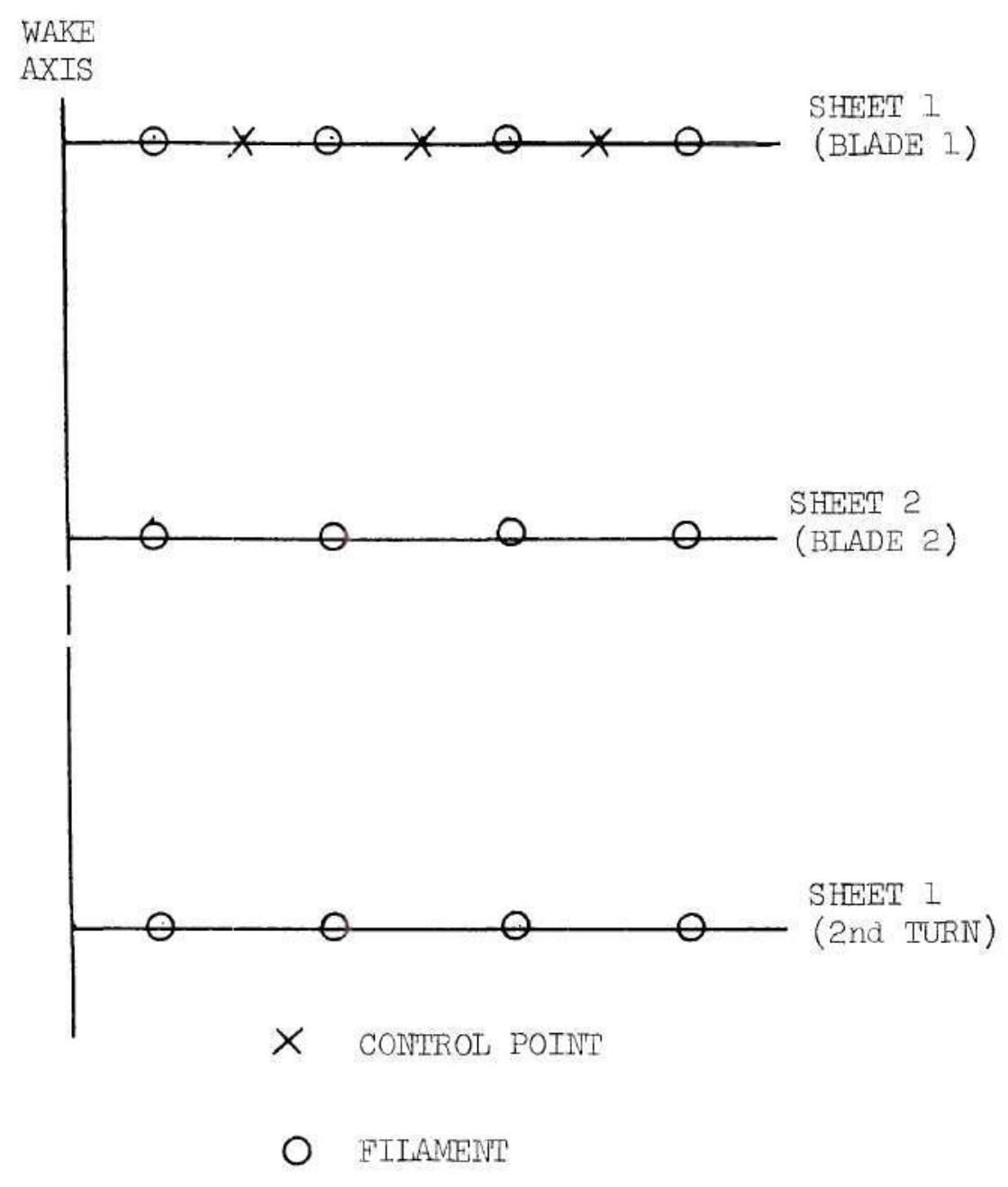

Figure 25. Schematic Diagram of the Arrangement of Filaments and Control Points in the Ultimate Wake of a Free Propeliter. 


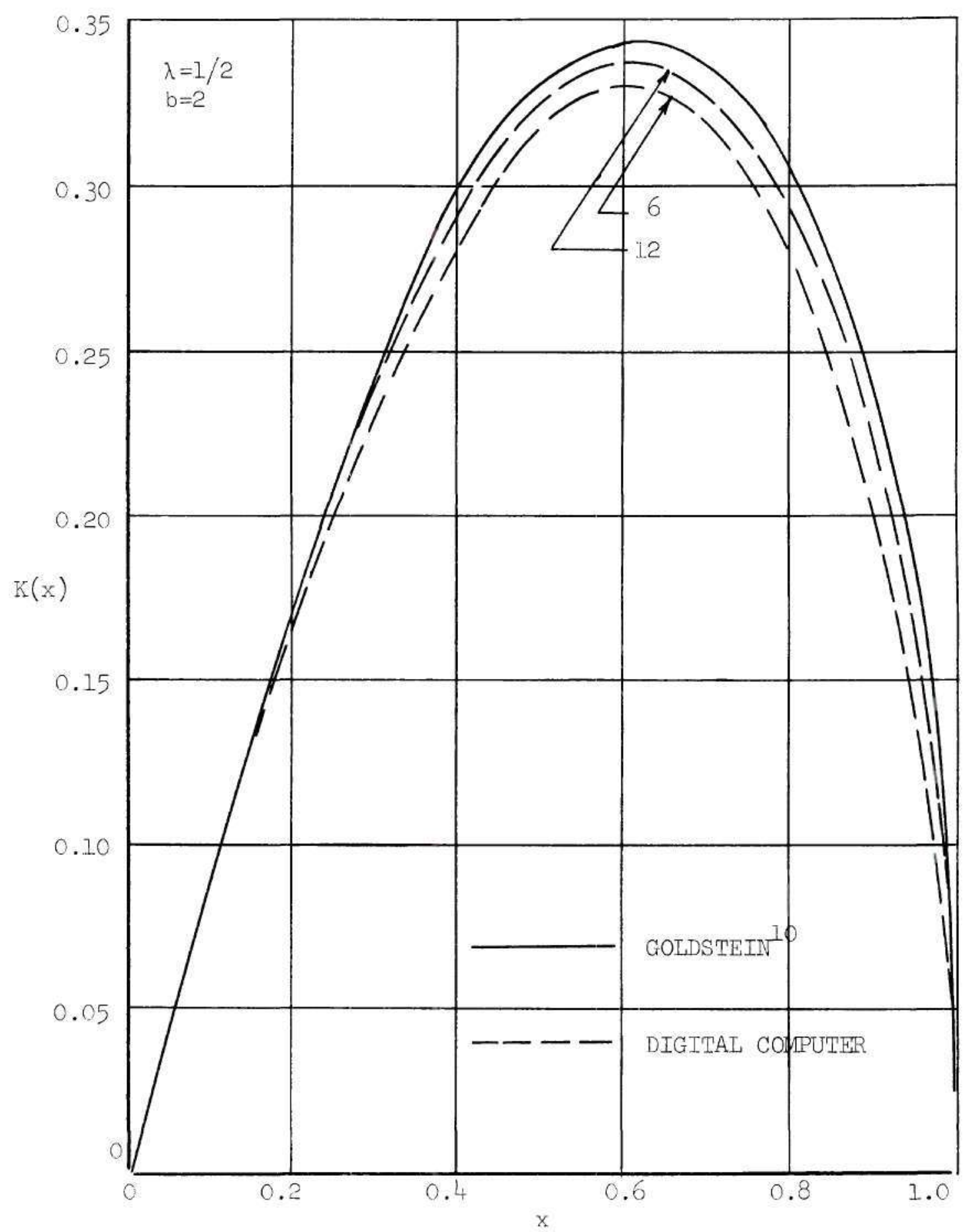

Figure 26. Comparison of Two Methods for Calculating the Blade Bound Vorticity for a Free Propeller. 


$$
\int_{0}^{1} \int_{0}^{1} \int_{0}^{2 \pi} \bar{u}_{z} \bar{r} d \bar{r} d \bar{z} d \Psi / 2 \pi=\frac{1}{2}\left[K+u_{\xi_{0}}\right]
$$

The comparisons are listed and reduced to a percent difference in Table I, where

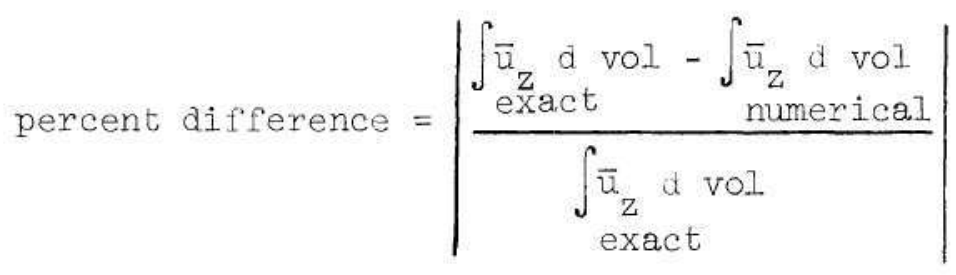

As the table shows, the difference in all cases was of the order of 1 percent or less so that the calculation and integration methods were considered to be sufficiently accurate.

As an additional check on the volume integrations, the values of $\epsilon_{r}, \epsilon_{\Psi}$ and $\epsilon_{z}$ were calculated for $\lambda=1$ and $b=2,4,8$, and 12 . These results are compared to the values for an infinite blade fan. As can be seen in Fig. 27 the results show satisfactcry convergence for increasing blade number. 
Table 1. Accuracy of the Wake Integrations

\begin{tabular}{|c|c|c|c|c|}
\hline$b$ & $\begin{array}{l}\int \bar{u}_{z} d \text { vol } \\
\text { numerical }\end{array}$ & $\begin{array}{l}\int \vec{u}_{z} d \text { vol } \\
\text { exact }\end{array}$ & Difference & $\%$ Difference \\
\hline 2 & .520 & .526 & .006 & 1.14 \\
\hline 3 & .552 & .557 & .005 & 0.90 \\
\hline 4 & .569 & .572 & .003 & 0.53 \\
\hline P & .589 & .590 & .001 & 0.17 \\
\hline
\end{tabular}




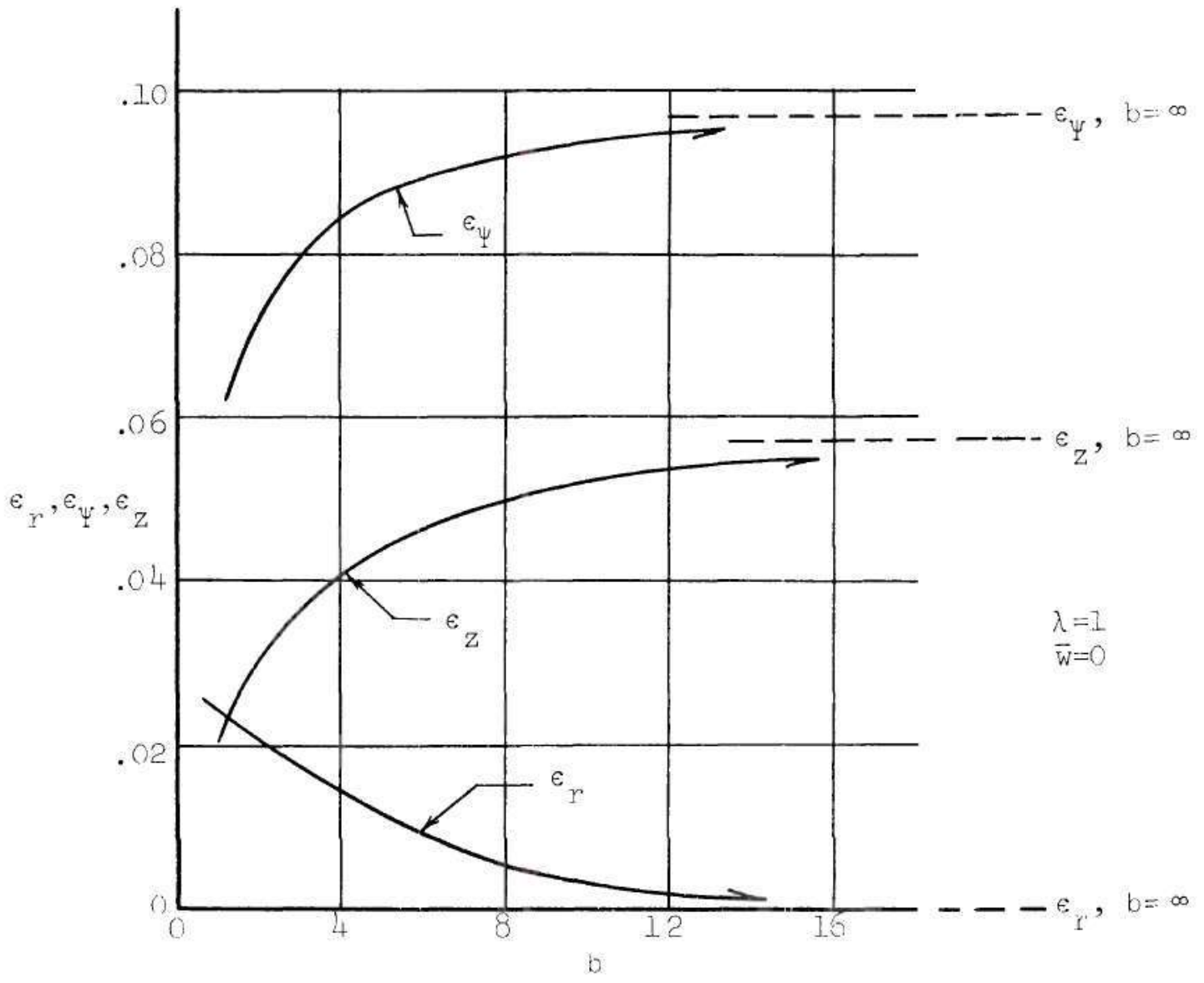

Figure 27. Convergence of the Wake Integration to the Exact Rosult; 13 for the Infinite Blade Case. 
APPENDIX IV

PERFORMANCE AND DESIGN PARAMETERS

\begin{tabular}{|c|c|c|c|c|c|c|}
\hline \multicolumn{3}{|c|}{$\lambda=1 / 8 \quad b=2$} & \multirow[b]{2}{*}{$c_{T}$} & \multirow[b]{2}{*}{$C_{p}$} & \multirow[b]{2}{*}{$C_{T_{P}} / C_{T}$} & \multirow[b]{2}{*}{$G$} \\
\hline$x$ & $K_{0}(x)$ & $\bar{w} / \lambda$ & & & & \\
\hline \multirow{2}{*}{0} & \multirow{2}{*}{0} & 0 & 0 & 0 & 1.0 & 1.0 \\
\hline & & .05 & 000719 & .0000877 & .9767 & $975 x$ \\
\hline \multirow{2}{*}{0.1} & \multirow{2}{*}{.4413} & .10 & .001435 & .0001705 & .9532 & .9507 \\
\hline & & .15 & .002148 & .0002486 & .9294 & .9260 \\
\hline \multirow{2}{*}{0.2} & \multirow{2}{*}{.6920} & 20 & .002858 & .0003219 & 2052 & .9012 \\
\hline & & 25 & .003567 & .0003904 & .8809 & .8765 \\
\hline \multirow{2}{*}{0.3} & \multirow{2}{*}{.8208} & .30 & .004275 & $.000 \times 5 \times 4$ & .8563 & .8517 \\
\hline & & .35 & .004982 & .0005317 & .8315 & .8268 \\
\hline \multirow{2}{*}{0.4} & \multirow{2}{*}{.8894} & 40 & .005689 & .0005685 & .8064 & .8020 \\
\hline & & 45 & .006396 & .0006187 & .7812 & .7771 \\
\hline \multirow{2}{*}{0.5} & \multirow{2}{*}{.9277} & .50 & .007105 & .0006645 & .7559 & .7522 \\
\hline & & .55 & .007815 & $.000>058$ & .7304 & .7272 \\
\hline \multirow{2}{*}{0.6} & \multirow{2}{*}{.9501} & 60 & .008527 & $.0007 * 27$ & .7047 & .7023 \\
\hline & & .65 & .009242 & .000775 & .6790 & .6773 \\
\hline \multirow{2}{*}{0.7} & \multirow[b]{2}{*}{.9637} & .70 & .009961 & .0008032 & .6532 & .6523 \\
\hline & & .75 & .01068 & .0008270 & 6273 & .6273 \\
\hline \multirow{2}{*}{0.8} & \multirow[b]{2}{*}{.9723} & .80 & 01141 & .0008464 & .6013 & .6022 \\
\hline & & .85 & .01214 & $.00086 / 4$ & .5753 & .5772 \\
\hline \multirow{2}{*}{0.9} & \multirow{2}{*}{9773} & .90 & .01288 & .0008722 & $.5 \times 93$ & .5515 \\
\hline & & 95 & .01362 & .0008786 & .5233 & .5270 \\
\hline \multirow{2}{*}{1.0} & \multirow{2}{*}{.9795} & 1.0 & .01437 & .0008808 & .4972 & 5019 \\
\hline & & & & & & \\
\hline
\end{tabular}




\begin{tabular}{|c|c|c|c|c|c|c|}
\hline$x$ & $K_{0}(x)$ & $\bar{w} / \lambda$ & $C_{T}$ & $C_{p}$ & $C_{T_{p}} / C_{T}$ & $G$ \\
\hline \multirow{2}{*}{0} & \multirow{2}{*}{0} & 0 & 0 & 0 & 1.0 & 1.0 \\
\hline & & .05 & .000723 & .0000881 & .9765 & \\
\hline \multirow{2}{*}{0.1} & \multirow{2}{*}{.4250} & .10 & .001443 & .0001714 & .9527 & \\
\hline & & .15 & .002160 & 0002498 & .9287 & \\
\hline \multirow{2}{*}{0.2} & \multirow{2}{*}{.7006} & .20 & .002875 & .0003234 & .9045 & \\
\hline & & .25 & .003588 & .0003923 & .8800 & \\
\hline \multirow{2}{*}{0.3} & \multirow{2}{*}{.8341} & .30 & $.00 \times 300$ & .0004566 & .8553 & \\
\hline & & .35 & .005012 & .0005162 & .8304 & \\
\hline \multirow[t]{2}{*}{0.4} & \multirow{2}{*}{.9001} & .40 & .005724 & .0005712 & .8054 & \\
\hline & & .45 & .006436 & .0006217 & .7802 & \\
\hline \multirow{2}{*}{0.5} & \multirow{2}{*}{.9352} & .50 & $.007 / 49$ & .0006677 & .7548 & \\
\hline & & .55 & .007864 & .0007092 & .7293 & \\
\hline \multirow{2}{*}{0.6} & \multirow{2}{*}{.9548} & .60 & .008581 & .0007463 & .7037 & \\
\hline & & .65 & .009300 & .0007789 & .6780 & \\
\hline \multirow{2}{*}{0.7} & \multirow{2}{*}{.9670} & .70 & .01002 & .0008071 & .6523 & \\
\hline & & .75 & .01075 & .0008310 & .6264 & \\
\hline \multirow{2}{*}{0.8} & \multirow{2}{*}{.9745} & .80 & $.011 \times 8$ & .0008505 & .6006 & \\
\hline & & .85 & .01221 & .0008656 & .5747 & \\
\hline \multirow{2}{*}{0.9} & \multirow{2}{*}{.978} & .90 & .01295 & .0008764 & .5487 & \\
\hline & & .95 & .01369 & .0008829 & .5228 & \\
\hline \multirow[t]{2}{*}{1.0} & \multirow[t]{2}{*}{.9818} & 1.0 & .01444 & -0008850 & 4969 & \\
\hline & & & & & & \\
\hline
\end{tabular}




\begin{tabular}{|c|c|c|c|c|c|c|}
\hline \multicolumn{3}{|c|}{$\lambda=1 / 8 \quad b=4$} & \multirow[b]{2}{*}{$C_{T}$} & \multirow[b]{2}{*}{$C_{p}$} & \multirow[b]{2}{*}{$C_{T_{p}} / C_{T}$} & \multirow[b]{2}{*}{$G$} \\
\hline$x$ & $K_{0}(x)$ & $\bar{w} / \lambda$ & & & & \\
\hline \multirow{2}{*}{0} & \multirow{2}{*}{0} & 0 & 0 & 0 & 1.0 & 1.0 \\
\hline & & .05 & .000724 & .0000883 & .9764 & \\
\hline \multirow{2}{*}{0.1} & \multirow[b]{2}{*}{.4130} & .10 & .001445 & .0001717 & .9526 & \\
\hline & & .15 & .002164 & .0002502 & .9285 & \\
\hline \multirow{2}{*}{0.2} & \multirow[b]{2}{*}{.7063} & 20 & .002881 & .0003240 & .9042 & \\
\hline & & 25 & .003596 & .0003930 & .8796 & \\
\hline \multirow{2}{*}{0.3} & \multirow{2}{*}{.8411} & .30 & .004310 & .0004574 & .8549 & \\
\hline & & .35 & .005023 & .0005171 & .8300 & \\
\hline \multirow{2}{*}{0.4} & \multirow{2}{*}{.9050} & .40 & .005737 & .0005723 & .8049 & \\
\hline & & .45 & .006451 & .0006289 & .7727 & \\
\hline \multirow{2}{*}{0.5} & \multirow[b]{2}{*}{.9378} & .50 & .007166 & .0006689 & .7544 & \\
\hline & & .55 & 007883 & .0007105 & .7289 & \\
\hline \multirow{2}{*}{0.6} & \multirow{2}{*}{.9564} & 60 & .008601 & .0007476 & .7033 & \\
\hline & & .65 & .009322 & .0007803 & 6777 & \\
\hline \multirow{2}{*}{0.7} & \multirow{2}{*}{.9678} & .70 & .01005 & .0008084 & .6519 & \\
\hline & & .75 & .01077 & .0008324 & .6261 & \\
\hline \multirow{2}{*}{0.8} & \multirow{2}{*}{.9752} & .80 & .01150 & .0008520 & .6003 & \\
\hline & & .85 & .01224 & .0008671 & .5744 & \\
\hline \multirow{2}{*}{0.9} & \multirow[b]{2}{*}{9801} & .90 & .01298 & $.0008>80$ & .5485 & \\
\hline & & .95 & .01372 & .0008845 & .5226 & \\
\hline \multirow{2}{*}{1.0} & \multirow{2}{*}{.9828} & 1.0 & .01447 & .0008866 & $\times 968$ & \\
\hline & & & & & & \\
\hline
\end{tabular}




\begin{tabular}{|c|c|c|c|c|c|c|}
\hline \multicolumn{3}{|c|}{$\lambda=1 / 4 \quad b=2$} & \multirow[b]{2}{*}{$C_{T}$} & \multirow[b]{2}{*}{$C_{p}$} & \multirow[b]{2}{*}{$C_{T_{p}} / C_{T}$} & \multirow[b]{2}{*}{$G$} \\
\hline$x$ & $K_{0}(x)$ & $\bar{w} / \lambda$ & & & & \\
\hline \multirow{2}{*}{0} & \multirow{2}{*}{0} & 0 & 0 & 0 & 1.0 & 1.0 \\
\hline & & .05 & .002453 & 000599 & .9802 & .2764 \\
\hline \multirow{2}{*}{0.1} & \multirow[b]{2}{*}{.2420} & .10 & .004874 & 001161 & .2596 & .9527 \\
\hline & & .15 & .007266 & 001687 & .9382 & .9288 \\
\hline \multirow{2}{*}{0.2} & \multirow{2}{*}{.4407} & 20 & .009636 & 002177 & .9160 & .9018 \\
\hline & & .25 & .01199 & 002633 & .8231 & 8807 \\
\hline \multirow{2}{*}{0.3} & \multirow{2}{*}{.5866} & 30 & .01433 & 003055 & .8695 & .8565 \\
\hline & & .35 & 01667 & 003445 & .8453 & 8321 \\
\hline \multirow{2}{*}{0.4} & \multirow{2}{*}{.6899} & 40 & .01901 & 003803 & .8204 & .8077 \\
\hline & & .45 & .02135 & 004129 & $.79+9$ & .7831 \\
\hline \multirow{2}{*}{0.5} & \multirow{2}{*}{.7626} & .50 & .02370 & 004425 & 7689 & .7585 \\
\hline & & .55 & .02607 & 004691 & .7425 & 7337 \\
\hline \multirow{2}{*}{0.6} & \multirow{2}{*}{.8139} & 60 & 02846 & 004927 & 7156 & 7089 \\
\hline & & .65 & .03088 & 005134 & .6884 & 6839 \\
\hline \multirow{2}{*}{0.7} & \multirow{2}{*}{.8500} & .70 & .03333 & 005313 & .6609 & 6589 \\
\hline & & .75 & .03582 & 005464 & .6331 & 6339 \\
\hline \multirow{2}{*}{0.8} & \multirow{2}{*}{.8747} & .80 & .03835 & 005586 & .6052 & 6087 \\
\hline & & .85 & .04094 & 005682 & .5771 & .5835 \\
\hline \multirow{2}{*}{0.9} & \multirow{2}{*}{.8899} & .90 & .04357 & 005750 & .5490 & .5582 \\
\hline & & .95 & .04627 & 005790 & .5208 & .5329 \\
\hline \multirow[t]{2}{*}{1.0} & \multirow{2}{*}{.8958} & 1.0 & .04903 & .005804 & 4927 & .5076 \\
\hline & & & & & & \\
\hline
\end{tabular}




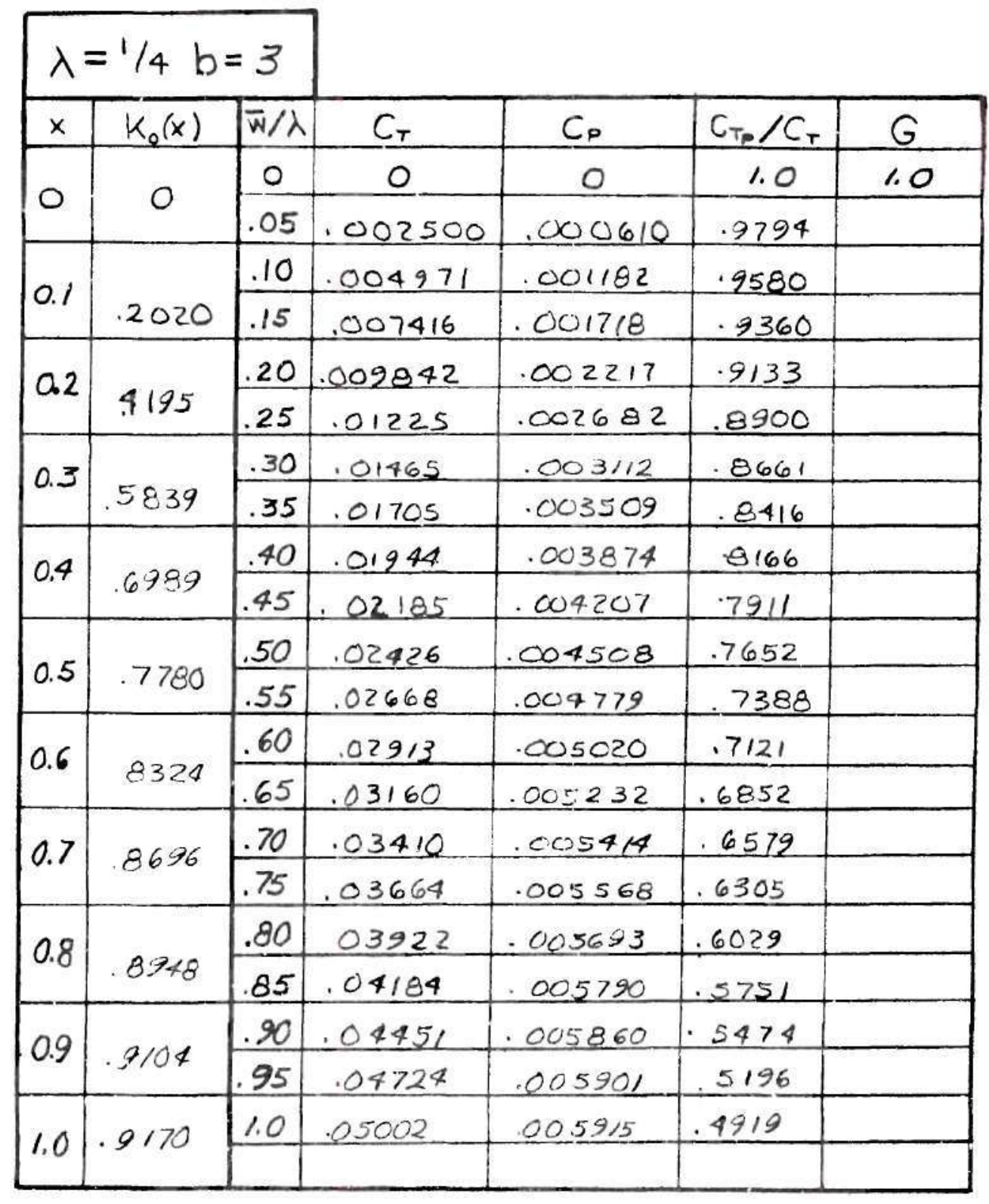




\begin{tabular}{|c|c|c|c|c|c|c|}
\hline \multicolumn{3}{|c|}{$\lambda=1 / 4 \quad b=4$} & \multirow[b]{2}{*}{$C_{T}$} & \multirow[b]{2}{*}{$C_{p}$} & \multirow[b]{2}{*}{$C_{T_{p}} / C_{T}$} & \multirow[b]{2}{*}{$G$} \\
\hline$x$ & $K_{0}(x)$ & $\bar{w} / \lambda$ & & & & \\
\hline \multirow{2}{*}{0} & \multirow{2}{*}{0} & 0 & 0 & 0 & 1.0 & 1.0 \\
\hline & & .05 & .002519 & .000614 & .2790 & \\
\hline \multirow{2}{*}{0.1} & \multirow[b]{2}{*}{.1783} & .10 & .005010 & .001191 & 9573 & \\
\hline & & .15 & .007478 & .001730 & .9350 & \\
\hline \multirow{2}{*}{0.2} & \multirow{2}{*}{4084} & .20 & .009927 & .002233 & .9121 & \\
\hline & & .25 & .01236 & .002701 & .8886 & \\
\hline \multirow{2}{*}{0.3} & \multirow[b]{2}{*}{.5838} & .30 & .01479 & .003135 & 8645 & \\
\hline & & .35 & .01721 & .003535 & .8399 & \\
\hline \multirow{2}{*}{0.4} & \multirow[b]{2}{*}{.7045} & .40 & .01963 & 003903 & .8149 & \\
\hline & & .45 & .02205 & .004238 & .7894 & \\
\hline \multirow{2}{*}{0.5} & \multirow[b]{2}{*}{.7856} & .50 & .02449 & 004542 & .7634 & \\
\hline & & .55 & .02694 & .004815 & .7371 & \\
\hline \multirow{2}{*}{0.6} & \multirow[b]{2}{*}{.8402} & .60 & .02941 & .005058 & 7105 & \\
\hline & & .65 & .03191 & .005271 & .6836 & \\
\hline \multirow{2}{*}{0.7} & \multirow[b]{2}{*}{.8772} & .70 & .03443 & .005455 & .6565 & \\
\hline & & .75 & .03698 & .005610 & .6292 & \\
\hline \multirow{2}{*}{0.8} & \multirow[b]{2}{*}{.9024} & .80 & .03958 & .005736 & .6017 & \\
\hline & & .85 & .04222 & .005834 & .5742 & \\
\hline \multirow{2}{*}{0.9} & \multirow[b]{2}{*}{.9185} & .90 & .04490 & .005904 & .5466 & \\
\hline & & .95 & .04764 & .005946 & 5190 & \\
\hline \multirow{2}{*}{1.0} & \multirow{2}{*}{.9258} & 1.0 & .05044 & .005960 & $49 / 4$ & \\
\hline & & & & & & \\
\hline
\end{tabular}




\begin{tabular}{|c|c|c|c|c|c|c|}
\hline \multicolumn{3}{|c|}{$\lambda=1 / 4 \quad b=6$} & \multirow[b]{2}{*}{$C_{T}$} & \multirow[b]{2}{*}{$C_{p}$} & \multirow[b]{2}{*}{$C_{T_{p}} / C_{T}$} & \multirow[b]{2}{*}{$G$} \\
\hline$x$ & $K_{0}(x)$ & $\bar{w} / \lambda$ & & & & \\
\hline \multirow{2}{*}{0} & \multirow{2}{*}{0} & 은 & 은 & 0 & 1.0 & 1.0 \\
\hline & & .05 & .002534 & .000618 & .9737 & \\
\hline \multirow{2}{*}{0.1} & \multirow[b]{2}{*}{.1492} & .10 & .005042 & 001198 & .9567 & \\
\hline & & .15 & .007527 & 001740 & .9341 & \\
\hline \multirow{2}{*}{0.2} & \multirow[b]{2}{*}{.4007} & 20 & .009995 & 002246 & 9110 & \\
\hline & & 25 & .01245 & .002717 & .8874 & \\
\hline \multirow{2}{*}{0.3} & \multirow[b]{2}{*}{.5905} & .30 & .01490 & .003154 & .8632 & \\
\hline & & .35 & .01734 & .003556 & .8385 & \\
\hline \multirow{2}{*}{0.4} & \multirow[b]{2}{*}{.7079} & 40 & .01978 & .003926 & 0134 & \\
\hline & & .45 & .02223 & .004263 & 7879 & \\
\hline \multirow{2}{*}{0.5} & \multirow[b]{2}{*}{.7890} & .50 & .02468 & .004569 & .7612 & \\
\hline & & .55 & .02715 & 004844 & .7357 & \\
\hline \multirow{2}{*}{0.6} & \multirow[b]{2}{*}{8508} & .60 & .02964 & 005089 & .7091 & \\
\hline & & .65 & .03215 & .005303 & .6823 & \\
\hline \multirow{2}{*}{0.7} & \multirow[b]{2}{*}{8851} & .70 & .03469 & .005488 & 6553 & \\
\hline & & .75 & .03727 & .005644 & .6281 & \\
\hline \multirow{2}{*}{0.8} & \multirow[b]{2}{*}{.9026} & .80 & .03988 & .005771 & .6008 & \\
\hline & & .85 & .04253 & .005870 & .5733 & \\
\hline \multirow{2}{*}{0.9} & \multirow{2}{*}{.9235} & .90 & .07523 & $.0059 \times 0$ & .5459 & \\
\hline & & .95 & .04797 & .005982 & .5184 & \\
\hline \multirow{2}{*}{1.0} & \multirow{2}{*}{.9381} & 1.0 & .05078 & .005996 & .4910 & \\
\hline & & & & & & \\
\hline
\end{tabular}




\begin{tabular}{|c|c|c|c|c|c|c|}
\hline \multicolumn{3}{|c|}{$\lambda=3 / 8 \quad b=2$} & \multirow[b]{2}{*}{$C_{T}$} & \multirow[b]{2}{*}{$C_{P}$} & \multirow[b]{2}{*}{$C_{T_{p}} / C_{T}$} & \multirow[b]{2}{*}{ G } \\
\hline$x$ & $K_{0}(x)$ & $\bar{w} / \lambda$ & & & & \\
\hline \multirow{2}{*}{0} & \multirow{2}{*}{0} & 0 & 0 & 0 & 1.0 & 1.0 \\
\hline & & .05 & $.00 \times 520$ & .001656 & .9832 & .9779 \\
\hline \multirow{2}{*}{0.1} & \multirow[b]{2}{*}{.1615} & .10 & .008944 & .003198 & .9652 & .9556 \\
\hline & & .15 & .01329 & .004629 & $.2 \times 61$ & .9330 \\
\hline \multirow{2}{*}{0.2} & \multirow{2}{*}{.3078} & .20 & .01757 & .005955 & .9257 & .9102 \\
\hline & & .25 & .02180 & .007178 & .9043 & .8871 \\
\hline \multirow{2}{*}{0.3} & \multirow{2}{*}{.4307} & .30 & .02600 & .008303 & .8816 & .8638 \\
\hline & & .35 & .03018 & .009333 & .8579 & $.8 \times 02$ \\
\hline \multirow{2}{*}{0.4} & \multirow{2}{*}{.5292} & .40 & $.03 \times 36$ & .01027 & .8331 & .8164 \\
\hline & & .45 & .03857 & .01112 & .8073 & .2924 \\
\hline \multirow{2}{*}{0.5} & \multirow[b]{2}{*}{.6059} & .50 & .04281 & .01182 & .7806 & .7682 \\
\hline & & .55 & .04710 & .01257 & .7530 & .7438 \\
\hline \multirow{2}{*}{0.6} & \multirow{2}{*}{.6614} & .60 & 05147 & .01318 & .7247 & .2191 \\
\hline & & .65 & .05593 & .01371 & .6957 & .6943 \\
\hline \multirow{2}{*}{0.7} & \multirow[b]{2}{*}{.7078} & .70 & .06050 & .01416 & .6662 & .6693 \\
\hline & & .75 & .06520 & .01254 & .6362 & .6442 \\
\hline \multirow{2}{*}{0.8} & \multirow{2}{*}{.7384} & .80 & .07005 & .01485 & .6058 & .6189 \\
\hline & & .85 & .07506 & .01509 & .5752 & .5935 \\
\hline \multirow{2}{*}{0.9} & \multirow[b]{2}{*}{.7575} & .90 & .08027 & .01526 & .5416 & .5679 \\
\hline & & .95 & .08568 & .01536 & .5139 & .5922 \\
\hline \multirow[t]{2}{*}{1.0} & \multirow{2}{*}{.7649} & 1.0 & .09132 & . 01539 & .9833 & .5162 \\
\hline & & & & & & \\
\hline
\end{tabular}




\begin{tabular}{|c|c|c|c|c|c|c|}
\hline \multicolumn{3}{|c|}{$\lambda=3 / 8 \quad b=3$} & \multirow[b]{2}{*}{$C_{T}$} & \multirow[b]{2}{*}{$C_{p}$} & \multirow[b]{2}{*}{$C_{T_{p}} / C_{T}$} & \multirow[b]{2}{*}{$G$} \\
\hline$x$ & $K_{0}(x)$ & $\bar{w} / \lambda$ & & & & \\
\hline \multirow{2}{*}{0} & \multirow{2}{*}{0} & 0 & 0 & 0 & 1.0 & 1.0 \\
\hline & & .05 & 1004698 & .001719 & .9827 & \\
\hline \multirow{2}{*}{0.1} & \multirow[b]{2}{*}{.1223} & .10 & .002295 & .003321 & 9643 & \\
\hline & & .15 & .01382 & .004808 & .9448 & \\
\hline \multirow{2}{*}{0.2} & \multirow{2}{*}{.2775} & .20 & .01827 & .006186 & .9242 & \\
\hline & & .25 & .02268 & .007459 & 9025 & \\
\hline \multirow[t]{2}{*}{0.3} & \multirow[b]{2}{*}{.4172} & .30 & .02706 & .008629 & .8798 & \\
\hline & & .35 & 0.3142 & .009701 & .8560 & \\
\hline \multirow{2}{*}{0.4} & \multirow{2}{*}{.5317} & .40 & .03578 & .01068 & .8312 & \\
\hline & & .45 & .04016 & .01157 & .8055 & \\
\hline \multirow{2}{*}{0.5} & \multirow{2}{*}{.6214} & .50 & .04457 & .01236 & .7790 & \\
\hline & & .55 & 04903 & .01308 & $\cdot 75 / 6$ & \\
\hline \multirow{2}{*}{0.6} & \multirow{2}{*}{.6898} & 60 & .05356 & .01371 & .7236 & \\
\hline & & .65 & .05818 & .01426 & .6999 & \\
\hline \multirow{2}{*}{0.7} & \multirow[b]{2}{*}{.7408} & .70 & .06291 & .01423 & .6657 & \\
\hline & & .75 & .06775 & .01513 & .0361 & \\
\hline \multirow{2}{*}{0.8} & \multirow{2}{*}{.7770} & .80 & .07274 & .01545 & .6062 & \\
\hline & & .85 & $.0>789$ & .01570 & .5761 & \\
\hline \multirow{2}{*}{0.9} & \multirow{2}{*}{.8002} & .90 & .08322 & 01588 & .5459 & \\
\hline & & .95 & .08874 & .01598 & .5156 & \\
\hline \multirow{2}{*}{1.0} & \multirow{2}{*}{.8097} & 1.0 & $.091 \times 8$ & 01602 & .4855 & \\
\hline & & & & & & \\
\hline
\end{tabular}




\begin{tabular}{|c|c|c|c|c|c|c|}
\hline \multicolumn{3}{|c|}{$\lambda=3 / 8 \quad b=4$} & \multirow[b]{2}{*}{$C_{T}$} & \multirow[b]{2}{*}{$C_{p}$} & \multirow[b]{2}{*}{$C_{T_{p}} / C_{T}$} & \multirow[b]{2}{*}{$G$} \\
\hline$x$ & $K_{0}(x)$ & $\overline{\mathrm{w}} / \lambda$ & & & & \\
\hline \multirow{2}{*}{0} & \multirow{2}{*}{0} & 0 & 0 & 0 & 1.0 & 1.0 \\
\hline & & .05 & .004773 & .001747 & 9819 & \\
\hline \multirow{2}{*}{0.1} & \multirow[b]{2}{*}{.1000} & .10 & .009459 & .003375 & .9629 & \\
\hline & & .15 & .01407 & 004887 & .9428 & \\
\hline \multirow{2}{*}{0.2} & \multirow{2}{*}{.2589} & 20 & .01862 & .006288 & .9216 & \\
\hline & & .25 & .02313 & .007582 & 8995 & \\
\hline \multirow{2}{*}{0.3} & \multirow{2}{*}{.4080} & .30 & .0 .2760 & .008772 & .8764 & \\
\hline & & .35 & .03206 & .002864 & .8524 & \\
\hline \multirow{2}{*}{0.4} & \multirow{2}{*}{$.53 R$} & .40 & 03653 & 01086 & .8275 & \\
\hline & & .45 & .04100 & .01176 & .8018 & \\
\hline \multirow{2}{*}{0.5} & \multirow{2}{*}{.6276} & .50 & .04552 & .01257 & .7753 & \\
\hline & & .55 & .05008 & .01330 & 7780 & \\
\hline \multirow[t]{2}{*}{0.6} & \multirow{2}{*}{.7010} & .60 & .05470 & $.0,394$ & 7202 & \\
\hline & & 65 & .05942 & .01450 & .6917 & \\
\hline \multirow[t]{2}{*}{0.7} & \multirow{2}{*}{.7556} & .70 & .06423 & .01499 & .6629 & \\
\hline & & .75 & .06916 & .01539 & .6336 & \\
\hline \multirow{2}{*}{0.8} & \multirow{2}{*}{.7948} & .80 & $.07 \times 22$ & .0 .572 & .6040 & \\
\hline & & .85 & .07914 & .01592 & .5743 & \\
\hline \multirow{2}{*}{0.9} & \multirow{2}{*}{.8203} & .90 & .08482 & .01615 & .5814 & \\
\hline & & .95 & $.090 \times 0$ & .01626 & .5186 & \\
\hline \multirow{2}{*}{1.0} & \multirow{2}{*}{$.83 A$} & 1.0 & .09618 & .01630 & .7848 & \\
\hline & & & & & & \\
\hline
\end{tabular}




\begin{tabular}{|c|c|c|c|c|c|c|}
\hline \multicolumn{3}{|c|}{$\lambda=3 / 8 \quad b=6$} & \multirow[b]{2}{*}{$C_{T}$} & \multirow[b]{2}{*}{$C_{p}$} & \multirow[b]{2}{*}{$C_{T_{p}} / C_{T}$} & \multirow[b]{2}{*}{$G$} \\
\hline$x$ & $K_{0}(x)$ & $\bar{w} / \lambda$ & & & & \\
\hline \multirow{2}{*}{0} & \multirow{2}{*}{0} & 0 & 0 & 0 & 1.0 & 1.0 \\
\hline & & .05 & .004837 & .001770 & .9816 & \\
\hline \multirow{2}{*}{0.1} & \multirow[b]{2}{*}{.07953} & .10 & .009589 & .003419 & 9623 & \\
\hline & & .15 & $.0,427$ & 004952 & 9419 & \\
\hline \multirow{2}{*}{0.2} & \multirow{2}{*}{.2409} & .20 & .01889 & .006372 & .9206 & \\
\hline & & .25 & .02346 & 007684 & .8983 & \\
\hline \multirow{2}{*}{0.3} & \multirow{2}{*}{.3991} & .30 & .02801 & .008892 & 8751 & \\
\hline & & .35 & .03254 & .01000 & .8511 & \\
\hline \multirow{2}{*}{0.4} & \multirow[b]{2}{*}{.5308} & .40 & .03708 & 01101 & .8261 & \\
\hline & & .45 & .04163 & .01192 & .8009 & \\
\hline \multirow{2}{*}{0.5} & \multirow{2}{*}{.6331} & .50 & .04621 & 01275 & .7739 & \\
\hline & & .55 & .05084 & .01349 & .7468 & \\
\hline \multirow{2}{*}{0.6} & \multirow[b]{2}{*}{.7103} & .60 & 05553 & .01414 & 7190 & \\
\hline & & .65 & .06031 & .01471 & .6907 & \\
\hline \multirow[t]{2}{*}{0.7} & \multirow{2}{*}{.7676} & .70 & .06518 & .01530 & .6620 & \\
\hline & & .75 & .07017 & .01561 & .6329 & \\
\hline \multirow{2}{*}{0.8} & \multirow{2}{*}{.8093} & .80 & .07529 & 01594 & .6039 & \\
\hline & & .85 & .08055 & .01620 & .5770 & \\
\hline \multirow{2}{*}{0.9} & \multirow[b]{2}{*}{.8375} & .90 & .08599 & .01638 & .5741 & \\
\hline & & .95 & .09160 & .01649 & $5|x\rangle$ & \\
\hline \multirow{2}{*}{1.0} & \multirow{2}{*}{.8512} & 1.0 & .09742 & .01653 & .8852 & \\
\hline & & & & & & \\
\hline
\end{tabular}




\begin{tabular}{|c|c|c|c|c|c|c|}
\hline \multicolumn{3}{|c|}{$\lambda=1 / 2 \quad b=2$} & \multirow[b]{2}{*}{$C_{T}$} & \multirow[b]{2}{*}{$C_{p}$} & \multirow[b]{2}{*}{$C_{T_{p}} / C_{T}$} & \multirow[b]{2}{*}{ G } \\
\hline$x$ & $K_{0}(x)$ & $\bar{w} / \lambda$ & & & & \\
\hline \multirow{2}{*}{0} & \multirow{2}{*}{0} & o & 0 & 0 & 1.0 & 1.0 \\
\hline & & .05 & .00648 & .003162 & 9854 & .9798 \\
\hline \multirow{2}{*}{0.1} & \multirow[b]{2}{*}{.1172} & .10 & .01279 & .006077 & -9694 & .9592 \\
\hline & & .15 & .01896 & .008754 & .9520 & .9382 \\
\hline \multirow{2}{*}{0.2} & \multirow[b]{2}{*}{2274} & 20 & .02500 & .01120 & .9332 & .9168 \\
\hline & & 25 & .03096 & .01343 & .9128 & .8950 \\
\hline \multirow{2}{*}{0.3} & \multirow{2}{*}{.3254} & .30 & .03685 & .01545 & .8910 & 8729 \\
\hline & & .35 & .04272 & .01728 & .8678 & .8503 \\
\hline \multirow{2}{*}{0.4} & \multirow{2}{*}{7090} & .40 & .04859 & .01891 & .8430 & .8274 \\
\hline & & .45 & .0 .5451 & .02036 & .8169 & .8091 \\
\hline \multirow{2}{*}{0.5} & \multirow{2}{*}{.4780} & .50 & .06050 & .02165 & .7895 & .7805 \\
\hline & & .55 & .06661 & 02277 & .7608 & .7565 \\
\hline \multirow{2}{*}{0.6} & \multirow{2}{*}{.5332} & .60 & .07287 & .02374 & 7309 & .7322 \\
\hline & & 65 & 07933 & .02456 & 7001 & .7075 \\
\hline \multirow[t]{2}{*}{0.7} & \multirow{2}{*}{.5757} & .70 & 08603 & .02525 & .6684 & .6826 \\
\hline & & .75 & .09301 & .02581 & .6360 & 6574 \\
\hline \multirow{2}{*}{0.8} & \multirow{2}{*}{.6067} & .80 & .1003 & .02625 & .6031 & .6319 \\
\hline & & .85 & .1080 & .02657 & .5699 & .6062 \\
\hline \multirow{2}{*}{0.9} & \multirow{2}{*}{.6266} & .90 & .1161 & .02678 & .5366 & .5803 \\
\hline & & .95 & .1247 & .02688 & .5032 & .5542 \\
\hline \multirow{2}{*}{1.0} & \multirow{2}{*}{.6349} & 1.0 & .1338 & 02688 & .4701 & .5279 \\
\hline & & & & & & \\
\hline
\end{tabular}




\begin{tabular}{|c|c|c|c|c|c|c|}
\hline \multicolumn{3}{|c|}{$\lambda=1 / 2 b=3$} & & & & \\
\hline$x$ & $K_{0}(x)$ & $\bar{w} / \lambda$ & $C_{T}$ & $C_{p}$ & $C_{T_{P}} / C_{T}$ & G \\
\hline \multirow{2}{*}{0} & \multirow{2}{*}{0} & 0 & 0 & 0 & 1.0 & 1.0 \\
\hline & & .05 & .00686 & .003342 & 9857 & \\
\hline \multirow{2}{*}{0.1} & \multirow[b]{2}{*}{.08357} & .10 & .01353 & .006420 & .9700 & \\
\hline & & .15 & .02004 & .009246 & .9528 & \\
\hline \multirow{2}{*}{0.2} & \multirow{2}{*}{.1979} & .20 & .02643 & .01183 & .9342 & \\
\hline & & .25 & .03272 & .01418 & .3112 & \\
\hline \multirow{2}{*}{0.3} & \multirow{2}{*}{.3099} & .30 & .03874 & .01632 & 8927 & \\
\hline & & .35 & .04513 & .01825 & .8697 & \\
\hline \multirow{2}{*}{0.4} & \multirow{2}{*}{.4095} & .40 & .05132 & .01998 & .8452 & \\
\hline & & 45 & .05754 & .02153 & 0194 & \\
\hline \multirow{2}{*}{0.5} & \multirow[b]{2}{*}{1935} & .50 & .06384 & .02290 & .7923 & \\
\hline & & .55 & .07025 & .02410 & .7640 & \\
\hline \multirow{2}{*}{0.6} & \multirow[b]{2}{*}{.5617} & .60 & .07681 & .02515 & $.73 \times 5$ & \\
\hline & & .65 & .08356 & .02604 & .2041 & \\
\hline \multirow[t]{2}{*}{0.7} & \multirow{2}{*}{.6150} & .70 & .09054 & .02679 & .6728 & \\
\hline & & .75 & .03780 & .02741 & .6708 & \\
\hline \multirow{2}{*}{0.8} & \multirow{2}{*}{6543} & .80 & .1054 & .02790 & .6084 & \\
\hline & & .85 & .1133 & $.0 ? 827$ & .5755 & \\
\hline \multirow{2}{*}{0.9} & \multirow{2}{*}{.6801} & .90 & .1217 & .02852 & .5426 & \\
\hline & & .95 & .1305 & 02865 & .5096 & \\
\hline \multirow[t]{2}{*}{1.0} & \multirow{2}{*}{.6912} & 1.0 & .1398 & .02867 & 4768 & \\
\hline & & & & & & \\
\hline
\end{tabular}




\begin{tabular}{|c|c|c|c|c|c|c|}
\hline \multicolumn{3}{|c|}{$\lambda=1 / 2 \quad b=4$} & \multirow[b]{2}{*}{$C_{T}$} & \multirow[b]{2}{*}{$C_{p}$} & \multirow[b]{2}{*}{$C_{T_{p}} / C_{T}$} & \multirow[b]{2}{*}{$G$} \\
\hline$x$ & $K_{0}(x)$ & $\bar{w} / \lambda$ & & & & \\
\hline \multirow{2}{*}{0} & \multirow{2}{*}{0} & 0 & 0 & 0 & 1.0 & 1.0 \\
\hline & & .05 & .00705 & .003438 & 9854 & \\
\hline \multirow{2}{*}{0.1} & \multirow[b]{2}{*}{.0648} & .10 & .01392 & .006606 & 9699 & \\
\hline & & .15 & .02063 & .009516 & .2520 & \\
\hline \multirow{2}{*}{0.2} & \multirow{2}{*}{.1791} & .20 & .02722 & .01218 & .9332 & \\
\hline & & .25 & .03370 & .01461 & .9130 & \\
\hline \multirow{2}{*}{0.3} & \multirow[b]{2}{*}{2979} & .30 & .04012 & .01682 & 8914 & \\
\hline & & .35 & .04650 & .01882 & .8684 & \\
\hline \multirow{2}{*}{0.4} & \multirow[b]{2}{*}{4065} & .40 & .05288 & .02062 & .8440 & \\
\hline & & .45 & .05929 & .02223 & .8183 & \\
\hline \multirow{2}{*}{0.5} & \multirow[b]{2}{*}{.4993} & .50 & .06578 & .02365 & .7913 & \\
\hline & & .55 & .07237 & .02491 & .7631 & \\
\hline \multirow{2}{*}{0.6} & \multirow[b]{2}{*}{5754} & .60 & .07911 & 02601 & .7339 & \\
\hline & & .65 & .08604 & .02695 & .7037 & \\
\hline \multirow[t]{2}{*}{0.7} & \multirow[b]{2}{*}{.6354} & .70 & .09319 & .02775 & .6727 & \\
\hline & & .75 & .1006 & .02841 & .6111 & \\
\hline \multirow{2}{*}{0.8} & \multirow[b]{2}{*}{.6803} & .80 & .1083 & 02894 & .6090 & \\
\hline & & .85 & .1164 & 0.2933 & .5765 & \\
\hline \multirow{2}{*}{0.9} & \multirow[b]{2}{*}{.7102} & .90 & .1249 & .02961 & .5439 & \\
\hline & & .95 & 1332 & .02976 & .5113 & \\
\hline \multirow{2}{*}{1.0} & \multirow{2}{*}{.7241} & 1.0 & .1733 & .02979 & .4788 & \\
\hline & & & & & & \\
\hline
\end{tabular}




\begin{tabular}{|c|c|c|c|c|c|c|}
\hline \multicolumn{3}{|c|}{$\lambda=1 / 2 \quad b=6$} & \multirow[b]{2}{*}{$C_{T}$} & \multirow[b]{2}{*}{$C_{p}$} & \multirow[b]{2}{*}{$C_{T_{p}} / C_{T}$} & \multirow[b]{2}{*}{$G$} \\
\hline$x$ & $K_{0}(x)$ & $\bar{w} / \lambda$ & & & & \\
\hline \multirow{2}{*}{0} & \multirow{2}{*}{0} & 0 & 0 & 0 & 1.0 & 1.0 \\
\hline & & .05 & .00720 & .003509 & 9847 & \\
\hline \multirow{2}{*}{0.1} & \multirow[b]{2}{*}{0482} & .10 & .01822 & .006747 & .9682 & \\
\hline & & .15 & .02109 & .009725 & .9504 & \\
\hline \multirow{2}{*}{0.2} & \multirow{2}{*}{.1588} & 20 & .02784 & .01246 & .9312 & \\
\hline & & .25 & .03449 & .01495 & .9106 & \\
\hline \multirow{2}{*}{0.3} & \multirow{2}{*}{2826} & .30 & .04107 & .01722 & .8888 & \\
\hline & & .35 & .04762 & .01928 & .8656 & \\
\hline \multirow{2}{*}{0.4} & \multirow{2}{*}{3992} & .40 & .05417 & .02114 & .8411 & \\
\hline & & .45 & .06075 & .02280 & .8153 & \\
\hline \multirow{2}{*}{0.5} & \multirow{2}{*}{5003} & .50 & .06740 & .02429 & .7884 & \\
\hline & & .55 & .07416 & .02560 & .7604 & \\
\hline \multirow{2}{*}{0.6} & \multirow[b]{2}{*}{5839} & 60 & 08106 & 02674 & $.73 / 4$ & \\
\hline & & .65 & $088 \mathrm{~A}$ & .02773 & .7015 & \\
\hline \multirow{2}{*}{0.7} & \multirow{2}{*}{.6505} & .70 & .09544 & .02857 & .6708 & \\
\hline & & .75 & .1030 & .02926 & .6396 & \\
\hline \multirow{2}{*}{0.8} & \multirow{2}{*}{7014} & .80 & .1109 & .02982 & .6078 & \\
\hline & & .85 & .1191 & .03025 & .5758 & \\
\hline \multirow{2}{*}{0.9} & \multirow{2}{*}{.7366} & .90 & .1277 & .03054 & $.5 \times 35$ & \\
\hline & & .95 & .1367 & .03071 & $.5 / 13$ & \\
\hline \multirow{2}{*}{1.0} & \multirow{2}{*}{.7542} & 1.0 & .1462 & .03076 & .4792 & \\
\hline & & & & & & \\
\hline
\end{tabular}




\begin{tabular}{|c|c|c|c|c|c|c|}
\hline \multicolumn{3}{|c|}{$\lambda=1 / 2 \quad b=8$} & \multirow[b]{2}{*}{$C_{T}$} & \multirow[b]{2}{*}{$C_{p}$} & \multirow[b]{2}{*}{$C_{T_{p}} / C_{T}$} & \multirow[b]{2}{*}{$G$} \\
\hline$x$ & $K_{0}(x)$ & $\bar{w} / \lambda$ & & & & \\
\hline \multirow{2}{*}{0} & \multirow{2}{*}{0} & 0 & 0 & 0 & 1.0 & 1.0 \\
\hline & & .05 & .00729 & .003550 & .9844 & \\
\hline \multirow{2}{*}{0.1} & \multirow[b]{2}{*}{.0425} & .10 & 01440 & .006829 & .9675 & \\
\hline & & .15 & .02136 & .009849 & .9493 & \\
\hline \multirow{2}{*}{0.2} & \multirow[b]{2}{*}{.1506} & 20 & .02820 & .01262 & .9299 & \\
\hline & & .25 & .03494 & $.015 / 6$ & .9092 & \\
\hline \multirow{2}{*}{0.3} & \multirow{2}{*}{2764} & .30 & .04162 & .01747 & .8871 & \\
\hline & & .35 & .04827 & .01956 & .8638 & \\
\hline \multirow{2}{*}{0.4} & \multirow[b]{2}{*}{.3968} & .40 & .05492 & .02116 & .8393 & \\
\hline & & .45 & .06160 & .02316 & .8135 & \\
\hline \multirow{2}{*}{0.5} & \multirow[b]{2}{*}{.5016} & .50 &, 06835 & $.02 \times 67$ & .7866 & \\
\hline & & .55 & .07520 & .02602 & .7587 & \\
\hline \multirow{2}{*}{0.6} & \multirow[b]{2}{*}{5884} & .60 & .08219 & .02712 & .7298 & \\
\hline & & .65 & .08936 & .02821 & .7000 & \\
\hline \multirow{2}{*}{0.7} & \multirow{2}{*}{.6579} & .70 & .09675 & .02907 & .7696 & \\
\hline & & .75 & 1044 & .02979 & .6385 & \\
\hline \multirow{2}{*}{0.8} & \multirow{2}{*}{.7119} & .80 & 1123 & .03037 & .6070 & \\
\hline & & .85 & .1206 & .03082 & .5751 & \\
\hline \multirow{2}{*}{0.9} & \multirow{2}{*}{.7507} & .90 & 1293 & .03113 & .5432 & \\
\hline & & .95 & .1384 & .03131 & 5112 & \\
\hline \multirow{2}{*}{1.0} & \multirow{2}{*}{.7720} & 1.0 & 1479 & .03136 & 4793 & \\
\hline & & & & & & \\
\hline
\end{tabular}




\begin{tabular}{|c|c|c|c|c|c|c|}
\hline \multicolumn{3}{|c|}{$\lambda=5 / 8 \quad b=2$} & \multirow[b]{2}{*}{$C_{T}$} & \multirow[b]{2}{*}{$C_{p}$} & \multirow[b]{2}{*}{$C_{T_{p}} / C_{T}$} & \multirow[b]{2}{*}{$G$} \\
\hline$x$ & $K_{0}(x)$ & $\bar{\omega} / \lambda$ & & & & \\
\hline \multirow{2}{*}{0} & \multirow{2}{*}{0} & 0 & 0 & 0 & 1.0 & 1.0 \\
\hline & & .05 & .008111 & .004943 & .9861 & 9818 \\
\hline \multirow{2}{*}{0.1} & \multirow[b]{2}{*}{.08808} & .10 & .01599 & .009487 & 9709 & 9630 \\
\hline & & .15 & .02366 & 01365 & .2542 & 2438 \\
\hline \multirow{2}{*}{0.2} & \multirow[b]{2}{*}{.1723} & 20 & $.03 / 18$ & .01745 & .9360 & .9240 \\
\hline & & .25 & .03856 & .02090 & 9162 & 9037 \\
\hline \multirow{2}{*}{0.3} & \multirow{2}{*}{.2495} & .30 & 04586 & 02402 & .8947 & 8828 \\
\hline & & .35 & 0.03313 & 02683 & .8715 & 8615 \\
\hline \multirow{2}{*}{0.4} & \multirow{2}{*}{.3178} & .40 & .06041 & .02935 & .8466 & 8396 \\
\hline & & .45 & 06776 & 03159 & 8201 & $8 / 72$ \\
\hline \multirow{2}{*}{0.5} & \multirow{2}{*}{3762} & .50 & 07525 & 03358 & .7918 & .7983 \\
\hline & & .55 & 08292 & 03532 & .7621 & .7709 \\
\hline \multirow{2}{*}{0.6} & \multirow{2}{*}{.4244} & .60 & 09085 & .03684 & 7308 & .7470 \\
\hline & & .65 & .09911 & .03814 & 6983 & .7227 \\
\hline \multirow{2}{*}{0.7} & \multirow[b]{2}{*}{.4627} & .70 & 1078 & .03923 & .6646 & .6979 \\
\hline & & .75 & -1169 & .04014 & .6301 & .6727 \\
\hline \multirow{2}{*}{0.8} & \multirow[b]{2}{*}{4912} & .80 & .1267 & .04087 & .5918 & .6470 \\
\hline & & .85 & 1370 & .0443 & .5592 & .620 \\
\hline \multirow{2}{*}{0.9} & \multirow{2}{*}{.5095} & .90 & 1482 & 0.0183 & .5238 & .5997 \\
\hline & & .95 & .1602 & .01206 & $48>7$ & .5681 \\
\hline \multirow{2}{*}{1.0} & \multirow{2}{*}{.5172} & 1.0 & .1731 & $012 \mathrm{H}$ & 1523 & .5411 \\
\hline & & & & & & \\
\hline
\end{tabular}




\begin{tabular}{|c|c|c|c|c|c|c|}
\hline \multicolumn{3}{|c|}{$\lambda=5 / 8 \quad b=3$} & \multirow[b]{2}{*}{$C_{T}$} & \multirow[b]{2}{*}{$C_{p}$} & \multirow[b]{2}{*}{$C_{T_{p}} / C_{T}$} & \multirow[b]{2}{*}{$G$} \\
\hline$x$ & $K_{0}(x)$ & $\bar{w} / \lambda$ & & & & \\
\hline \multirow{2}{*}{0} & \multirow{2}{*}{0} & 0 & 0 & 0 & 1.0 & 1.0 \\
\hline & & .05 & .008731 & .005331 & .9878 & \\
\hline \multirow{2}{*}{0.1} & \multirow{2}{*}{.06086} & .10 & .01718 & .01023 & .9742 & \\
\hline & & .15 & .02540 & .01473 & .2590 & \\
\hline \multirow{2}{*}{0.2} & \multirow{2}{*}{.1477} & .20 & .03341 & .01883 & .9421 & \\
\hline & & .25 & .04128 & 02257 & .9235 & \\
\hline \multirow{2}{*}{0.3} & \multirow{2}{*}{2366} & .30 & .04903 & .02595 & .2032 & \\
\hline & & .35 & .05673 & .02900 & .8810 & \\
\hline \multirow{2}{*}{0.4} & \multirow{2}{*}{.3195} & .40 & .06443 & .03173 & .8569 & \\
\hline & & .45 & .07219 & 03417 & .8311 & \\
\hline \multirow{2}{*}{0.5} & \multirow[b]{2}{*}{.3926} & .50 & .08007 & .03633 & .8035 & \\
\hline & & .55 & .08814 & .03822 & .7742 & \\
\hline \multirow{2}{*}{0.6} & \multirow[b]{2}{*}{.4542} & .60 & .09676 & 03987 & .7434 & \\
\hline & & .65 & .1051 & $.04 / 29$ & 7111 & \\
\hline \multirow{2}{*}{0.7} & \multirow{2}{*}{.5080} & .70 & .1142 & .04249 & .6772 & \\
\hline & & .75 & .1237 & .04348 & .6133 & \\
\hline \multirow{2}{*}{0.8} & \multirow{2}{*}{.5417} & .80 & .1338 & .04428 & .6082 & \\
\hline & & .85 & .1446 & .04989 & .5726 & \\
\hline \multirow{2}{*}{0.9} & \multirow{2}{*}{.5668} & .90 & 1561 & .04532 & .5367 & \\
\hline & & .95 & 1685 & .04557 & .5009 & \\
\hline \multirow[t]{2}{*}{1.0} & \multirow{2}{*}{.5774} & 1.0 & 1817 & .04566 & .7655 & \\
\hline & & & & & & \\
\hline
\end{tabular}




\begin{tabular}{|c|c|c|c|c|c|c|}
\hline \multicolumn{3}{|c|}{$\lambda=5 / 8 \quad b=4$} & \multirow[b]{2}{*}{$C_{T}$} & \multirow[b]{2}{*}{$C_{p}$} & \multirow[b]{2}{*}{$C_{T_{p}} / C_{T}$} & \multirow[b]{2}{*}{$G$} \\
\hline$x$ & $K_{0}(x)$ & $\bar{\omega} / \lambda$ & & & & \\
\hline \multirow{2}{*}{0} & \multirow{2}{*}{0} & 0 & 0 & 0 & 1.0 & 1.0 \\
\hline & & .05 & .002045 & .005525 & .9881 & \\
\hline \multirow{2}{*}{0.1} & \multirow{2}{*}{.04530} & .10 & .01780 & .01061 & 9746 & \\
\hline & & .15 & .02631 & .01527 & 9576 & \\
\hline \multirow{2}{*}{0.2} & \multirow{2}{*}{.1301} & 20 & .03461 & .01953 & 9429 & \\
\hline & & .25 & .04274 & .02341 & 2245 & \\
\hline \multirow{2}{*}{0.3} & \multirow[b]{2}{*}{2239} & .30 & .05077 & .02692 & 2044 & \\
\hline & & .35 & .05873 & .03009 & 8824 & \\
\hline \multirow{2}{*}{0.4} & \multirow[b]{2}{*}{3148} & .40 & .06669 & .03294 & 8585 & \\
\hline & & .45 & .07470 & .03547 & 8329 & \\
\hline \multirow{2}{*}{0.5} & \multirow{2}{*}{.3968} & .50 & 08283 & .03772 & 8055 & \\
\hline & & .55 & .09114 & .03969 & 7765 & \\
\hline \multirow{2}{*}{0.6} & \multirow{2}{*}{.4672} & .60 & .09970 & .04141 & 7760 & \\
\hline & & .65 & .086 & .04289 & 2180 & \\
\hline \multirow{2}{*}{0.7} & \multirow{2}{*}{.5249} & .70 & .1179 & .04414 & 6809 & \\
\hline & & .75 & 1277 & .01518 & 6468 & \\
\hline \multirow{2}{*}{0.8} & \multirow{2}{*}{.5692} & .80 & .1380 & .04601 & 6119 & \\
\hline & & .85 & .1490 & .04665 & 5766 & \\
\hline \multirow{2}{*}{0.9} & \multirow{2}{*}{5994} & .90 & .1607 & .04710 & 5410 & \\
\hline & & .95 & .1732 & .04737 & 5054 & \\
\hline \multirow[t]{2}{*}{1.0} & \multirow[t]{2}{*}{.6128} & 1.0 & .1867 & .04746 & 4701 & \\
\hline & & & & & & \\
\hline
\end{tabular}




\begin{tabular}{|c|c|c|c|c|c|c|}
\hline \multicolumn{3}{|c|}{$\lambda=5 / 8 \quad b=6$} & \multirow[b]{2}{*}{$C_{T}$} & \multirow[b]{2}{*}{$C_{p}$} & \multirow[b]{2}{*}{$C_{T_{p}} / C_{T}$} & \multirow[b]{2}{*}{$G$} \\
\hline$x$ & $K_{0}(x)$ & $\bar{w} / \lambda$ & & & & \\
\hline \multirow{2}{*}{0} & \multirow{2}{*}{0} & 0 & 0 & 0 & 1.0 & 1.0 \\
\hline & & .05 & .002357 & .005703 & .9858 & \\
\hline \multirow{2}{*}{0.1} & \multirow[b]{2}{*}{.03219} & .10 & .01876 & .01095 & .9704 & \\
\hline & & .15 & .02733 & .01577 & .9535 & \\
\hline \multirow{2}{*}{0.2} & \multirow[b]{2}{*}{.1117} & 20 & .03603 & .02018 & .9352 & \\
\hline & & .25 & .04458 & .02419 & .9154 & \\
\hline \multirow{2}{*}{0.3} & \multirow[b]{2}{*}{.2080} & .30 & .05303 & .02783 & $.89 \times 1$ & \\
\hline & & .35 & $.06 / 43$ & .03111 & .8713 & \\
\hline \multirow{2}{*}{0.4} & \multirow[b]{2}{*}{3058} & .40 & .06783 & $.03 \times 06$ & .8769 & \\
\hline & & .45 & .07829 & 03669 & 8210 & \\
\hline \multirow{2}{*}{0.5} & \multirow{2}{*}{.3967} & .50 & .08686 & .03902 & .7936 & \\
\hline & & .55 & .09561 & .04107 & $.76 \times 8$ & \\
\hline \multirow[t]{2}{*}{0.6} & \multirow{2}{*}{.4765} & .60 & .1046 & .04286 & .7347 & \\
\hline & & .65 & .1139 & .01439 & .7035 & \\
\hline \multirow{2}{*}{0.7} & \multirow{2}{*}{.5434} & .70 & .1236 & .04570 & .6713 & \\
\hline & & .75 & 1337 & .04677 & .6382 & \\
\hline \multirow{2}{*}{0.8} & \multirow{2}{*}{.5962} & .80 & .1414 & .04764 & .6045 & \\
\hline & & .85 & .1557 & .04831 & .5703 & \\
\hline \multirow{2}{*}{0.9} & \multirow{2}{*}{.6334} & .90 & .1676 & .04878 & .5360 & \\
\hline & & .95 & .1801 & .04906 & .5016 & \\
\hline \multirow[t]{2}{*}{1.0} & \multirow{2}{*}{.6512} & 1.0 & $.19 \times 1$ & .04915 & .4645 & \\
\hline & & & & & & \\
\hline
\end{tabular}




\begin{tabular}{|c|c|c|c|c|c|c|}
\hline \multicolumn{3}{|c|}{$\lambda=5 / 8 b=8$} & \multirow[b]{2}{*}{$C_{T}$} & \multirow[b]{2}{*}{$C_{p}$} & \multirow[b]{2}{*}{$C_{T_{p}} / C_{T}$} & \multirow[b]{2}{*}{$G$} \\
\hline$x$ & $K_{0}(x)$ & $\bar{w} / \lambda$ & & & & \\
\hline \multirow{2}{*}{0} & \multirow{2}{*}{0} & 0 & 0 & 0 & 1.0 & 1.0 \\
\hline & & .05 & 0 & .005780 & 9874 & \\
\hline \multirow{2}{*}{0.1} & \multirow[b]{2}{*}{.02782} & .10 & .009467 & .01110 & .9733 & \\
\hline & & .15 & .02758 & .01599 & .9577 & \\
\hline \multirow[t]{2}{*}{0.2} & \multirow{2}{*}{.1036} & 20 & 03631 & .02046 & .9705 & \\
\hline & & .25 & 04487 & .02452 & .9218 & \\
\hline \multirow{2}{*}{0.3} & \multirow{2}{*}{1999} & .30 & .05332 & .02822 & .9013 & \\
\hline & & .35 & .06171 & .03155 & .8791 & \\
\hline \multirow{2}{*}{0.4} & \multirow{2}{*}{.3003} & 40 & .07010 & .03454 & .8553 & \\
\hline & & .45 & .07853 & .03721 & .8297 & \\
\hline \multirow{2}{*}{0.5} & \multirow{2}{*}{3950} & .50 & 08707 & .03958 & 8026 & \\
\hline & & .55 & .09580 & .04167 & 7739 & \\
\hline \multirow{2}{*}{0.6} & \multirow{2}{*}{.4790} & .60 & 1048 & .04348 & .7437 & \\
\hline & & .65 & -1140 & .04503 & .7123 & \\
\hline \multirow{2}{*}{0.7} & \multirow{2}{*}{.5503} & .70 & .1237 & .04637 & .6798 & \\
\hline & & .75 & 1339 & .04747 & .6464 & \\
\hline \multirow{2}{*}{0.8} & \multirow{2}{*}{.6078} & .80 & .1445 & .04835 & .6122 & \\
\hline & & .85 & 1558 & 04903 & .5776 & \\
\hline \multirow{2}{*}{0.9} & \multirow{2}{*}{.6498} & .90 & .1679 & .04950 & $.5 \times 28$ & \\
\hline & & .95 & .1807 & .04979 & .5079 & \\
\hline \multirow[t]{2}{*}{1.0} & \multirow{2}{*}{.6711} & 1.0 & 1941 & .04988 & .4732 & \\
\hline & & & & & & \\
\hline
\end{tabular}




\begin{tabular}{|c|c|c|c|c|c|c|}
\hline \multicolumn{3}{|c|}{$\lambda=3 / 4 \quad b=2$} & \multirow[b]{2}{*}{$C_{T}$} & \multirow[b]{2}{*}{$C_{p}$} & \multirow[b]{2}{*}{$C_{T_{P}} / C_{T}$} & \multirow[b]{2}{*}{$G$} \\
\hline$x$ & $K_{0}(x)$ & $\bar{w} / \lambda$ & & & & \\
\hline \multirow{2}{*}{0} & \multirow{2}{*}{0} & 0 & 은 & 0 & 1.0 & 1.0 \\
\hline & & .05 & .00944 & .00682 & .9867 & 9837 \\
\hline \multirow{2}{*}{0.1} & \multirow[b]{2}{*}{.0677} & .10 & .01859 & .01319 & .9720 & .9668 \\
\hline & & .15 & .02749 & .01893 & 9559 & .9423 \\
\hline \multirow{2}{*}{0.2} & \multirow[b]{2}{*}{.1340} & .20 & .03619 & .02414 & .9382 & .9312 \\
\hline & & .25 & .04473 & 02883 & .9188 & .9125 \\
\hline \multirow{2}{*}{0.3} & \multirow[b]{2}{*}{1963} & .30 & .05316 & .03305 & .8977 & .8931 \\
\hline & & .35 & .06156 & .03681 & .8747 & .8730 \\
\hline \multirow{2}{*}{0.4} & \multirow[b]{2}{*}{.2516} & .40 & .06997 & .04015 & 8498 & .8523 \\
\hline & & .45 & .07849 & .04309 & .8229 & 8309 \\
\hline \multirow{2}{*}{0.5} & \multirow{2}{*}{2995} & .50 & .08719 & .01567 & .7940 & .8089 \\
\hline & & .55 & .09616 & .04790 & .7633 & .7862 \\
\hline \multirow{2}{*}{0.6} & \multirow{2}{*}{.3406} & 60 & .1055 & .04982 & .7308 & .7628 \\
\hline & & .65 & .1153 & .05145 & .6967 & .7389 \\
\hline \multirow[t]{2}{*}{0.7} & \multirow[b]{2}{*}{.3746} & .70 & .1258 & .05280 & .6611 & .7143 \\
\hline & & .75 & .1369 & .05392 & .6244 & .6891 \\
\hline \multirow{2}{*}{0.8} & \multirow{2}{*}{.4002} & .80 & .1490 & .05480 & .5869 & .6634 \\
\hline & & .85 & .1620 & .05547 & .5488 & .6371 \\
\hline \multirow{2}{*}{0.9} & \multirow{2}{*}{$4 / 61$} & .90 & .1762 & .05594 & 5106 & .6103 \\
\hline & & .95 & .1918 & .05622 & 4725 & .5832 \\
\hline \multirow{2}{*}{1.0} & \multirow{2}{*}{.4227} & 1.0 & 2088 & .05631 & 4350 & .5556 \\
\hline & & & & & & \\
\hline
\end{tabular}




\begin{tabular}{|c|c|c|c|c|c|c|}
\hline \multicolumn{3}{|c|}{$\lambda=3 / 4 \quad b=3$} & \multirow[b]{2}{*}{$C_{T}$} & \multirow[b]{2}{*}{$C_{p}$} & \multirow[b]{2}{*}{$C_{T_{p}} / C_{T}$} & \multirow[b]{2}{*}{$G$} \\
\hline$x$ & $K_{0}(x)$ & $\bar{w} / \lambda$ & & & & \\
\hline \multirow{2}{*}{0} & \multirow{2}{*}{0} & ㅇ & ㅇ & 0 & 1.0 & 1.0 \\
\hline & & .05 & .01030 & .00754 & .9895 & \\
\hline \multirow{2}{*}{0.1} & \multirow[b]{2}{*}{.0463} & .10 & .02023 & .01444 & .9776 & \\
\hline & & .15 & .02984 & .02074 & .9640 & \\
\hline \multirow{2}{*}{0.2} & \multirow[b]{2}{*}{.1140} & 20 & .03919 & .02645 & .9486 & \\
\hline & & .25 & .04832 & .03161 & .2313 & \\
\hline \multirow{2}{*}{0.3} & \multirow[b]{2}{*}{.1853} & .30 & .05731 & .03624 & .2121 & \\
\hline & & .35 & .06621 & .01038 & .8907 & \\
\hline \multirow{2}{*}{0.4} & \multirow[b]{2}{*}{.2536} & 40 & .07512 & .04406 & .8672 & \\
\hline & & .45 & .08410 & .04731 & $.01 / 4$ & \\
\hline \multirow{2}{*}{0.5} & \multirow[b]{2}{*}{.3156} & .50 & .09326 & .05015 & 8135 & \\
\hline & & .55 & .1027 & .05262 & .7835 & \\
\hline \multirow{2}{*}{0.6} & \multirow{2}{*}{.3694} & 60 & 1125 & .05474 & $75 \mathrm{H}$ & \\
\hline & & .65 & 1228 & .05655 & .7176 & \\
\hline \multirow{2}{*}{0.7} & \multirow[b]{2}{*}{.4138} & .70 & .1337 & .05806 & .6821 & \\
\hline & & .75 & .1453 & .05929 & 6453 & \\
\hline \multirow{2}{*}{0.8} & \multirow{2}{*}{4482} & .80 & 1578 & 06028 & 6075 & \\
\hline & & .85 & .1713 & .06102 & .5690 & \\
\hline \multirow{2}{*}{0.9} & \multirow{2}{*}{.4715} & .90 & .1861 & .06155 & .5303 & \\
\hline & & .95 & .2021 & .06186 & .4917 & \\
\hline \multirow{2}{*}{1.0} & \multirow{2}{*}{.4816} & 1.0 & 2196 & .06196 & .8535 & \\
\hline & & & & & & \\
\hline
\end{tabular}




\begin{tabular}{|c|c|c|c|c|c|c|}
\hline \multicolumn{3}{|c|}{$\lambda=3 / 4 \quad b=4$} & \multirow[b]{2}{*}{$C_{T}$} & \multirow[b]{2}{*}{$C_{p}$} & \multirow[b]{2}{*}{$C_{T_{p}} / C_{T}$} & \multirow[b]{2}{*}{$G$} \\
\hline$x$ & $K_{0}(x)$ & $\bar{w} / \lambda$ & & & & \\
\hline \multirow{2}{*}{0} & \multirow{2}{*}{0} & 0 & 0 & 0 & 1.0 & 1.0 \\
\hline & & .05 & .01075 & 00788 & .9902 & \\
\hline \multirow{2}{*}{0.1} & \multirow[b]{2}{*}{.0321} & .10 & .02111 & 01510 & .9788 & \\
\hline & & .15 & .03113 & .02168 & .9658 & \\
\hline \multirow{2}{*}{0.2} & \multirow[b]{2}{*}{.1016} & 20 & 04085 & .02766 & .9510 & \\
\hline & & .25 & 05035 & .03306 & .9343 & \\
\hline \multirow{2}{*}{0.3} & \multirow[b]{2}{*}{.1704} & .30 & .05969 & 03792 & $.9 / 56$ & \\
\hline & & .35 & .06893 & .04226 & .8947 & \\
\hline \multirow{2}{*}{0.4} & \multirow[b]{2}{*}{.2531} & .40 & .07816 & .04612 & $.87 / 6$ & \\
\hline & & .45 & 08745 & .04953 & .8462 & \\
\hline \multirow{2}{*}{0.5} & \multirow{2}{*}{.3160} & .50 & 09692 & .05252 & .8187 & \\
\hline & & .55 & .1066 & .05512 & .7890 & \\
\hline \multirow[t]{2}{*}{0.6} & \multirow[b]{2}{*}{.3851} & .60 & .1167 & .05736 & .7573 & \\
\hline & & .65 & .1273 & .05926 & .7237 & \\
\hline \multirow{2}{*}{0.7} & \multirow{2}{*}{4323} & .70 & .1385 & .06085 & .6885 & \\
\hline & & .75 & .1505 & .06216 & .6519 & \\
\hline \multirow{2}{*}{0.8} & \multirow[b]{2}{*}{.4773} & .80 & .1633 & .06320 & .6143 & \\
\hline & & .85 & .1771 & .06398 & .5760 & \\
\hline \multirow{2}{*}{0.9} & \multirow[b]{2}{*}{.5035} & .90 & .1921 & .06454 & .5374 & \\
\hline & & .95 & .2084 & .06487 & 4988 & \\
\hline \multirow{2}{*}{1.0} & \multirow{2}{*}{.5180} & 1.0 & .2262 & 06498 & .4607 & \\
\hline & & & & & & \\
\hline
\end{tabular}




\begin{tabular}{|c|c|c|c|c|c|c|}
\hline \multicolumn{3}{|c|}{$\lambda=3 / 4 \quad b=6$} & \multirow[b]{2}{*}{$C_{T}$} & \multirow[b]{2}{*}{$C_{p}$} & \multirow[b]{2}{*}{$C_{T_{p}} / C_{T}$} & \multirow[b]{2}{*}{$G$} \\
\hline$x$ & $K_{0}(x)$ & $\bar{w} / \lambda$ & & & & \\
\hline \multirow{2}{*}{0} & \multirow{2}{*}{0} & 0 & 0 & 0 & 1.0 & 1.0 \\
\hline & & .05 & .01119 & .00821 & .9903 & \\
\hline \multirow{2}{*}{0.1} & \multirow{2}{*}{.02301} & .10 & .02198 & .01573 & .9791 & \\
\hline & & .15 & .03240 & .02259 & .9662 & \\
\hline \multirow{2}{*}{0.2} & \multirow{2}{*}{08265} & 20 & .04253 & .02883 & .9516 & \\
\hline & & .25 & .05242 & .03447 & .9350 & \\
\hline \multirow{2}{*}{0.3} & \multirow{2}{*}{.1596} & .30 & .06213 & .03954 & .9164 & \\
\hline & & .35 & .07174 & .04409 & .8957 & \\
\hline \multirow{2}{*}{0.4} & \multirow{2}{*}{.2397} & .40 & 08133 & .04813 & 8728 & \\
\hline & & .45 & .09099 & .05170 & 8777 & \\
\hline \multirow{2}{*}{0.5} & \multirow{2}{*}{.3187} & .50 & .1008 & .05484 & 8205 & \\
\hline & & .55 & .1109 & .05756 & .7911 & \\
\hline \multirow{2}{*}{0.6} & \multirow{2}{*}{.3909} & .60 & .1213 & .05991 & .7597 & \\
\hline & & .65 & .1322 & .06191 & .7265 & \\
\hline \multirow{2}{*}{0.7} & \multirow{2}{*}{.4535} & .70 & .1438 & .06359 & .6917 & \\
\hline & & .75 & .1560 & .06496 & .6555 & \\
\hline \multirow{2}{*}{0.8} & \multirow{2}{*}{.5042} & .80 & 1692 & 06606 & .6183 & \\
\hline & & .85 & 1833 & .06689 & .5804 & \\
\hline \multirow{2}{*}{0.9} & \multirow{2}{*}{$.5 \not 07$} & .90 & 1986 & . 06747 & .5121 & \\
\hline & & .95 & 2152 & .06782 & .5039 & \\
\hline \multirow{2}{*}{1.0} & \multirow{2}{*}{.5582} & 1.0 & .2333 & .06794 & 4660 & \\
\hline & & & & & & \\
\hline
\end{tabular}




\begin{tabular}{|c|c|c|c|c|c|c|}
\hline \multicolumn{3}{|c|}{$\lambda=3 / 4 \quad b=8$} & \multirow[b]{2}{*}{$C_{T}$} & \multirow[b]{2}{*}{$C_{p}$} & \multirow[b]{2}{*}{$C_{T_{p}} / C_{T}$} & \multirow[b]{2}{*}{$G$} \\
\hline$x$ & $K_{0}(x)$ & $\bar{w} / \lambda$ & & & & \\
\hline \multirow{2}{*}{0} & \multirow{2}{*}{0} & 0 & 0 & 0 & 1.0 & 1.0 \\
\hline & & .05 & .01139 & .00835 & 9900 & \\
\hline \multirow{2}{*}{0.1} & \multirow[b]{2}{*}{01960} & .10 & .02238 & .01601 & .9785 & \\
\hline & & .15 & .03300 & .02300 & .9654 & \\
\hline \multirow{2}{*}{0.2} & \multirow[b]{2}{*}{07545} & .20 & .04333 & .02935 & .9506 & \\
\hline & & .25 & .05342 & .03510 & .2338 & \\
\hline \multirow{2}{*}{0.3} & \multirow{2}{*}{.1506} & .30 & .06333 & .04027 & .9151 & \\
\hline & & .35 & .07314 & .04490 & .8943 & \\
\hline \multirow{2}{*}{0.4} & \multirow{2}{*}{.2334} & .40 & .08293 & .04903 & 8214 & \\
\hline & & .45 & .09278 & .05267 & .8464 & \\
\hline \multirow{2}{*}{0.5} & \multirow{2}{*}{$3 / 58$} & .50 & .1028 & .05587 & 8192 & \\
\hline & & .55 & .1131 & .05866 & .7900 & \\
\hline \multirow{2}{*}{0.6} & \multirow{2}{*}{.3927} & .60 & .1237 & .06106 & .7588 & \\
\hline & & .65 & .1348 & .06311 & .7258 & \\
\hline \multirow{2}{*}{0.7} & \multirow{2}{*}{.4606} & .70 & .1465 & .06482 & .6915 & \\
\hline & & .75 & 1589 & .06623 & .6554 & \\
\hline \multirow{2}{*}{0.8} & \multirow{2}{*}{.5171} & .80 & .1722 & .06735 & .6185 & \\
\hline & & .85 & 1865 & .06820 & .5809 & \\
\hline \multirow{2}{*}{0.9} & \multirow{2}{*}{.5591} & .90 & 2020 & .06880 & .5429 & \\
\hline & & .95 & 2187 & .06916 & .5049 & \\
\hline \multirow{2}{*}{1.0} & \multirow{2}{*}{.5805} & 1.0 & .2369 & .06928 & .4673 & \\
\hline & & & & & & \\
\hline
\end{tabular}




\begin{tabular}{|c|c|c|c|c|c|c|}
\hline \multicolumn{3}{|c|}{$\lambda=3 / 4 \quad b=12$} & \multirow[b]{2}{*}{$C_{T}$} & \multirow[b]{2}{*}{$C_{p}$} & \multirow[b]{2}{*}{$C_{T_{p}} / C_{T}$} & \multirow[b]{2}{*}{$G$} \\
\hline$x$ & $K_{0}(x)$ & $\bar{w} / \lambda$ & & & & \\
\hline \multirow{2}{*}{0} & \multirow{2}{*}{0} & 0 & 0 & 0 & 1.0 & 1.0 \\
\hline & & .05 & .01156 & .00847 & 9896 & \\
\hline \multirow{2}{*}{0.1} & \multirow{2}{*}{.01784} & .10 & .02272 & .01624 & .9777 & \\
\hline & & .15 & .03353 & .02333 & 9642 & \\
\hline \multirow{2}{*}{0.2} & \multirow[b]{2}{*}{.06979} & 20 & 04404 & .02978 & .9489 & \\
\hline & & 25 & .05431 & .03562 & .9319 & \\
\hline \multirow{2}{*}{0.3} & \multirow{2}{*}{.1436} & 30 & .06441 & .04088 & .9129 & \\
\hline & & .35 & .07442 & .04559 & .8920 & \\
\hline \multirow{2}{*}{0.4} & \multirow{2}{*}{2272} & .40 & .08440 & .04978 & .8690 & \\
\hline & & .45 & .09444 & .05349 & .8439 & \\
\hline \multirow{2}{*}{0.5} & \multirow{2}{*}{.3120} & .50 & .1046 & .05675 & 8167 & \\
\hline & & .55 & .1151 & .05959 & .7876 & \\
\hline \multirow{2}{*}{0.6} & \multirow{2}{*}{.3923} & .60 & .1259 & .06203 & .7566 & \\
\hline & & .65 & .1372 & $.064 / 2$ & .7238 & \\
\hline \multirow{2}{*}{0.7} & \multirow{2}{*}{.4649} & .70 & .1431 & .06587 & .6896 & \\
\hline & & .75 & .1617 & .06730 & .6540 & \\
\hline \multirow{2}{*}{0.8} & \multirow{2}{*}{.5272} & .80 & .1751 & .06845 & .6175 & \\
\hline & & .85 & .1896 & .06932 & 5802 & \\
\hline \multirow{2}{*}{0.9} & \multirow{2}{*}{5762} & .90 & .2051 & .06993 & .5127 & \\
\hline & & .95 & 2220 & .07029 & .5050 & \\
\hline \multirow{2}{*}{1.0} & \multirow{2}{*}{.6038} & 1.0 & 2702 & .07041 & .1677 & \\
\hline & & & & & & \\
\hline
\end{tabular}




\begin{tabular}{|c|c|c|c|c|c|c|}
\hline \multicolumn{7}{|c|}{$\lambda=1 \quad b=2$} \\
\hline$x$ & $K_{0}(x)$ & $\overline{\mathrm{w}} / \lambda$ & $C_{T}$ & $C_{p}$ & $C_{T_{p}} / C_{T}$ & $G$ \\
\hline \multirow{2}{*}{0} & \multirow{2}{*}{0} & 0 & 0 & 0 & 1.0 & 1.0 \\
\hline & & .05 & 01131 & .01099 & .9877 & .9872 \\
\hline \multirow{2}{*}{0.1} & \multirow[b]{2}{*}{.04382} & .10 & .02227 & .02098 & .9742 & 9737 \\
\hline & & .15 & .03290 & .02999 & .2594 & .9595 \\
\hline \multirow[t]{2}{*}{0.2} & \multirow[b]{2}{*}{08653} & 20 & .04325 & .03808 & .2430 & .9416 \\
\hline & & .25 & .05339 & .04529 & .9248 & .9289 \\
\hline \multirow{2}{*}{0.3} & \multirow[b]{2}{*}{.1271} & .30 & .06337 & $.05 / 67$ & .9047 & .9124 \\
\hline & & .35 & .07329 & 05726 & .8825 &, 8951 \\
\hline \multirow{2}{*}{0.4} & \multirow[b]{2}{*}{.1647} & .40 & 08325 & .06212 & .8580 & .8769 \\
\hline & & .45 & 09334 & .06631 & .8311 & .8578 \\
\hline \multirow{2}{*}{0.5} & \multirow[b]{2}{*}{.1985} & .50 & .1037 & .06987 & .8017 & 8377 \\
\hline & & .55 & .1145 & .07287 & .7696 & .8167 \\
\hline \multirow{2}{*}{0.6} & \multirow{2}{*}{.2280} & 60 & .1252 & .07536 & .7351 & .7987 \\
\hline & & .65 & 1381 & 07780 & .6982 & .7718 \\
\hline \multirow{2}{*}{0.7} & \multirow{2}{*}{.2526} & .70 & .1514 & .07909 & .6591 & .7479 \\
\hline & & .75 & 1659 & 08034 & .6182 & .7230 \\
\hline \multirow{2}{*}{0.8} & \multirow[b]{2}{*}{.2719} & .80 & 1821 & 08133 & .5759 & .6972 \\
\hline & & .85 & 2001 & .08205 & .5329 & .6705 \\
\hline \multirow{2}{*}{0.9} & \multirow{2}{*}{2850} & .90 & 2203 & .08255 & .4896 & .6430 \\
\hline & & .95 & 2432 & .08283 & .4467 & .6178 \\
\hline \multirow{2}{*}{1.0} & \multirow{2}{*}{2910} & 1.0 & 2690 & .08293 & .4047 & .5858 \\
\hline & & & & & & \\
\hline
\end{tabular}




\begin{tabular}{|c|c|c|c|c|c|c|}
\hline \multicolumn{3}{|c|}{$\lambda=1 \quad b=3$} & \multirow[b]{2}{*}{$C_{T}$} & \multirow[b]{2}{*}{$C_{p}$} & \multirow[b]{2}{*}{$C_{T_{p}} / C_{T}$} & \multirow[b]{2}{*}{$G$} \\
\hline$x$ & $K_{0}(x)$ & $\bar{w} / \lambda$ & & & & \\
\hline \multirow{2}{*}{0} & \multirow{2}{*}{0} & 0 & 0 & 0 & 1.0 & 10 \\
\hline & & .05 & .01255 & .01224 & .2917 & \\
\hline \multirow{2}{*}{0.1} & \multirow[b]{2}{*}{.02915} & .10 & .02460 & .02337 & 9820 & \\
\hline & & .15 & .03621 & .03342 & 9208 & \\
\hline \multirow{2}{*}{0.2} & \multirow[b]{2}{*}{.07297} & 20 & .04743 & .04246 & .9578 & \\
\hline & & .25 & .05835 & .05051 & .2128 & \\
\hline \multirow{2}{*}{0.3} & \multirow[b]{2}{*}{.1204} & .30 & 06903 & .05764 & .9256 & \\
\hline & & .35 & .07958 & .06391 & .9059 & \\
\hline \multirow{2}{*}{0.4} & \multirow[b]{2}{*}{.1673} & 40 & .09010 & .06936 & .83837 & \\
\hline & & .45 & .1007 & .07106 & 8586 & \\
\hline \multirow{2}{*}{0.5} & \multirow{2}{*}{.2113} & .50 & .1116 & .07807 & .8306 & \\
\hline & & .55 & .1229 & .08145 & .7996 & \\
\hline \multirow[t]{2}{*}{0.6} & \multirow[b]{2}{*}{.2506} & .60 & .1348 & .08427 & .7657 & \\
\hline & & .65 & 1475 & .08658 & .7290 & \\
\hline \multirow{2}{*}{0.7} & \multirow[b]{2}{*}{2841} & .70 & .1613 & .08844 & .6899 & \\
\hline & & .75 & .1764 & .08991 & .6785 & \\
\hline \multirow{2}{*}{0.8} & \multirow{2}{*}{.3108} & .80 & 1932 & .09104 & .6056 & \\
\hline & & .85 & 2119 & .09187 & .5615 & \\
\hline \multirow{2}{*}{0.9} & \multirow{2}{*}{3294} & .90 & 2328 & .09244 & .5170 & \\
\hline & & .95 & 2564 & .03277 & .4728 & \\
\hline \multirow{2}{*}{1.0} & \multirow{2}{*}{.3382} & 1.0 & 2830 & .09288 & .4234 & \\
\hline & & & & & & \\
\hline
\end{tabular}




\begin{tabular}{|c|c|c|c|c|c|c|}
\hline \multicolumn{3}{|c|}{$\lambda=1 \quad b=4$} & \multirow[b]{2}{*}{$C_{T}$} & \multirow[b]{2}{*}{$C_{p}$} & \multirow[b]{2}{*}{$C_{T_{p}} / C_{T}$} & \multirow[b]{2}{*}{$G$} \\
\hline$x$ & $K_{0}(x)$ & $\bar{w} / \lambda$ & & & & \\
\hline \multirow{2}{*}{0} & \multirow{2}{*}{0} & 0 & 0 & 0 & 1.0 & 1.0 \\
\hline & & .05 & .01329 & .01299 & .9233 & \\
\hline \multirow{2}{*}{0.1} & \multirow[b]{2}{*}{02018} & .10 & .02602 & .02480 & .9852 & \\
\hline & & .15 & .03824 & $.0354 y$ & .9755 & \\
\hline \multirow{2}{*}{0.2} & \multirow[b]{2}{*}{.06226} & .20 & .05002 & .04508 & .9610 & \\
\hline & & .25 & .06144 & .05365 & .2504 & \\
\hline \multirow{2}{*}{0.3} & \multirow{2}{*}{.1122} & .30 & .07258 & .06125 & .9345 & \\
\hline & & .35 & .08356 & .06792 & .9160 & \\
\hline \multirow{2}{*}{0.4} & \multirow[b]{2}{*}{.1645} & .40 & .09448 & .07373 & .8948 & \\
\hline & & 45 & .1055 & .07875 & .8706 & \\
\hline \multirow{2}{*}{0.5} & \multirow{2}{*}{.2154} & .50 & 1167 & 08304 & 8434 & \\
\hline & & .55 & .1284 & .08666 & .8130 & \\
\hline \multirow{2}{*}{0.6} & \multirow[b]{2}{*}{.2621} & .60 & .1807 & 0.8968 & .7796 & \\
\hline & & .65 & 1537 & .02216 & .7432 & \\
\hline \multirow{2}{*}{0.7} & \multirow[b]{2}{*}{.3028} & .70 & .1619 & .09416 & .7041 & \\
\hline & & .75 & 1834 & .09575 & .6628 & \\
\hline \multirow{2}{*}{0.8} & \multirow[b]{2}{*}{$.3350 \overline{0}$} & .80 & .2006 & .09697 & .6197 & \\
\hline & & .85 & 2197 & .09787 & .5755 & \\
\hline \multirow{2}{*}{0.9} & \multirow[b]{2}{*}{$.35 M$} & .90 & 2710 & .09848 & .5306 & \\
\hline & & .95 & 2651 & .09884 & .7859 & \\
\hline \multirow{2}{*}{1.0} & \multirow{2}{*}{.3708} & 1.0 & .2921 & 07896 & $.4 \times 20$ & \\
\hline & & & & & & \\
\hline
\end{tabular}




\begin{tabular}{|c|c|c|c|c|c|c|}
\hline \multicolumn{3}{|c|}{$\lambda=1 \quad b=6$} & \multirow[b]{2}{*}{$C_{T}$} & \multirow[b]{2}{*}{$C_{p}$} & \multirow[b]{2}{*}{$C_{T_{p}} / C_{T}$} & \multirow[b]{2}{*}{$G$} \\
\hline$x$ & $K_{0}(x)$ & $\bar{w} / \lambda$ & & & & \\
\hline \multirow{2}{*}{0} & \multirow{2}{*}{0} & 0 & 0 & 0 & 1.0 & 10 \\
\hline & & .05 & .01401 & .01371 & .921 & \\
\hline \multirow{2}{*}{0.1} & \multirow[b]{2}{*}{.01341} & .10 & .02741 & .02618 & .2868 & \\
\hline & & .15 & .04026 & .03747 & .9779 & \\
\hline \multirow{2}{*}{0.2} & \multirow[b]{2}{*}{.05020} & 20 & .05262 & .04762 & .9671 & \\
\hline & & 25 & .06459 & .05668 & .9543 & \\
\hline \multirow{2}{*}{0.3} & \multirow{2}{*}{.09991} & .30 & .07626 & 06473 & 9390 & \\
\hline & & .35 & .08773 & .07180 & .2213 & \\
\hline \multirow{2}{*}{0.4} & \multirow{2}{*}{.1562} & .40 & .09916 & .07798 & .9007 & \\
\hline & & .45 & .1106 & .08331 & 8771 & \\
\hline \multirow{2}{*}{0.5} & \multirow{2}{*}{2140} & .50 & .1223 & .09787 & .8505 & \\
\hline & & .55 & .1344 & .09172 & .8206 & \\
\hline \multirow{2}{*}{0.6} & \multirow{2}{*}{.2695} & .60 & 1471 & .09496 & .7877 & \\
\hline & & .65 & .1606 & .09762 & .2518 & \\
\hline \multirow{2}{*}{0.7} & \multirow{2}{*}{3198} & .70 & .1752 & 09977 & .7131 & \\
\hline & & .75 & .1912 & .1015 & .6721 & \\
\hline \multirow{2}{*}{0.8} & \multirow{2}{*}{3619} & .80 & 2087 & .1028 & .6293 & \\
\hline & & .85 & 2283 & .1038 & .5852 & \\
\hline \multirow{2}{*}{0.9} & \multirow{2}{*}{.3930} & .90 & .2501 & .1044 & .5402 & \\
\hline & & .95 & .2746 & 1048 & 4258 & \\
\hline \multirow[t]{2}{*}{1.0} & \multirow{2}{*}{.4088} & 1.0 & 3021 & 1049 & .7517 & \\
\hline & & & & & & \\
\hline
\end{tabular}




\begin{tabular}{|c|c|c|c|c|c|c|}
\hline \multicolumn{3}{|c|}{$\lambda=1 \quad b=8$} & \multirow[b]{2}{*}{$C_{T}$} & \multirow[b]{2}{*}{$C_{p}$} & \multirow[b]{2}{*}{$C_{T_{p}} / C_{T}$} & \multirow[b]{2}{*}{$G$} \\
\hline$x$ & $K_{0}(x)$ & $\bar{w} / \lambda$ & & & & \\
\hline \multirow{2}{*}{0} & \multirow{2}{*}{0} & 0 & 0 & 0 & 1.0 & 1.0 \\
\hline & & .05 & .01438 & .01406 & .9942 & \\
\hline \multirow{2}{*}{0.1} & \multirow[b]{2}{*}{.01123} & .10 & .02812 & .02687 & .9869 & \\
\hline & & .15 & .04131 & .03816 & .9781 & \\
\hline \multirow{2}{*}{0.2} & \multirow[b]{2}{*}{.04485} & .20 & .05400 & .04888 & .9673 & \\
\hline & & .25 & .06628 & .05820 & .9545 & \\
\hline \multirow{2}{*}{0.3} & \multirow[b]{2}{*}{.09312} & .30 & .02824 & .06648 & .9394 & \\
\hline & & .35 & .09001 & .07376 & .9217 & \\
\hline \multirow{2}{*}{0.4} & \multirow[b]{2}{*}{.1502} & 40 & .1017 & .08011 & .9013 & \\
\hline & & .45 & .1134 & .08561 & .8779 & \\
\hline \multirow{2}{*}{0.5} & \multirow[b]{2}{*}{2109} & .50 & .1254 & .09032 & .8514 & \\
\hline & & .55 & .1378 & $.09 \times 30$ & .8218 & \\
\hline \multirow{2}{*}{0.6} & \multirow[b]{2}{*}{.2709} & 60 & .1507 & .09764 & .7892 & \\
\hline & & 65 & .1645 & $: 1004$ & .7535 & \\
\hline \multirow{2}{*}{0.7} & \multirow[b]{2}{*}{.3267} & .70 & .1794 & .1026 & .7152 & \\
\hline & & .75 & .1956 & .1044 & .6245 & \\
\hline \multirow{2}{*}{0.8} & \multirow[b]{2}{*}{.3751} & .80 & .2135 & .1057 & .6320 & \\
\hline & & .85 & .2333 & .1067 & .5882 & \\
\hline \multirow{2}{*}{0.9} & \multirow{2}{*}{.4121} & .90 & .2553 & $.107 x$ & .5438 & \\
\hline & & .95 & .2801 & .1078 & 4993 & \\
\hline \multirow{2}{*}{1.0} & \multirow{2}{*}{.4320} & 1.0 & 3078 & 1080 & .4554 & \\
\hline & & & & & & \\
\hline
\end{tabular}




\begin{tabular}{|c|c|c|c|c|c|c|}
\hline \multicolumn{3}{|c|}{$\lambda=1 \quad b=12$} & \multirow[b]{2}{*}{$C_{T}$} & \multirow[b]{2}{*}{$C_{p}$} & \multirow[b]{2}{*}{$C_{T_{p}} / C_{T}$} & \multirow[b]{2}{*}{$G$} \\
\hline$x$ & $k_{0}(x)$ & $\bar{w} / \lambda$ & & & & \\
\hline \multirow{2}{*}{0} & \multirow{2}{*}{0} & 0 & 0 & 0 & 1.0 & 1.0 \\
\hline & & .05 & .01465 & .01428 & .2939 & \\
\hline \multirow{2}{*}{0.1} & \multirow[b]{2}{*}{.01019} & .10 & .02867 & .02719 & .9863 & \\
\hline & & .15 & .04213 & .03878 & .9771 & \\
\hline \multirow{2}{*}{0.2} & \multirow{2}{*}{.01091} & 20 & .05509 & .04913 & .9662 & \\
\hline & & .25 & .06764 & .05830 & .9531 & \\
\hline \multirow{2}{*}{0.3} & \multirow{2}{*}{.08740} & .30 & .07988 & .06637 & .2378 & \\
\hline & & .35 & .09192 & .07340 & 9200 & \\
\hline \multirow{2}{*}{0.4} & \multirow{2}{*}{.1441} & 40 & .1039 & .07264 & .8995 & \\
\hline & & .45 & .1159 & .08464 & .8761 & \\
\hline \multirow{2}{*}{0.5} & \multirow[b]{2}{*}{.2061} & .50 & .1281 & .08900 & .8497 & \\
\hline & & .55 & .1407 & .09262 & .8202 & \\
\hline \multirow{2}{*}{0.6} & \multirow[b]{2}{*}{.2693} & 60 & .1540 & .09558 & .7878 & \\
\hline & & .65 & .1680 & .09193 & .7524 & \\
\hline \multirow{2}{*}{0.7} & \multirow[b]{2}{*}{.3302} & .70 & .1831 & 09976 & .7145 & \\
\hline & & .75 & .1226 & .1011 & .6742 & \\
\hline \multirow{2}{*}{0.8} & \multirow{2}{*}{.3850} & .80 & .2177 & $10<1$ & .6321 & \\
\hline & & .85 & .2377 & .1027 & .5887 & \\
\hline \multirow{2}{*}{0.9} & \multirow{2}{*}{4289} & 90 & .2600 & 1030 & .5447 & \\
\hline & & .95 & .2849 & .1030 & .5006 & \\
\hline \multirow{2}{*}{1.0} & \multirow{2}{*}{.4542} & 1.0 & 3127 & .1028 & .4570 & \\
\hline & & & & & & \\
\hline
\end{tabular}




\begin{tabular}{|c|c|c|c|c|c|c|}
\hline \multicolumn{3}{|c|}{$\lambda=1 \quad b=16$} & \multirow[b]{2}{*}{$C_{T}$} & \multirow[b]{2}{*}{$C_{p}$} & \multirow[b]{2}{*}{$C_{T_{p}} / C_{T}$} & \multirow[b]{2}{*}{$G$} \\
\hline$x$ & $K_{0}(x)$ & $\bar{w} / \lambda$ & & & & \\
\hline \multirow{2}{*}{0} & \multirow{2}{*}{0} & 0 & 0 & 0 & 1.0 & 10 \\
\hline & & .05 & .01479 & .01446 & 9936 & \\
\hline \multirow{2}{*}{0.1} & \multirow[b]{2}{*}{.00997} & .10 & .02896 & .02764 & .9857 & \\
\hline & & .15 & .04256 & .03957 & .9763 & \\
\hline \multirow{2}{*}{0.2} & \multirow[b]{2}{*}{.03950} & 20 & .05568 & .05031 & 9651 & \\
\hline & & .25 & .06838 & .05992 & .9518 & \\
\hline \multirow{2}{*}{0.3} & \multirow[b]{2}{*}{.08512} & .30 & .08077 & .06845 & 9363 & \\
\hline & & .35 & .09296 & .07597 & 9183 & \\
\hline \multirow{2}{*}{0.4} & \multirow{2}{*}{$.14 / 5$} & .40 & .1051 & .08254 & 8977 & \\
\hline & & .45 & .1172 & 08823 & $87 \times 3$ & \\
\hline \multirow{2}{*}{0.5} & \multirow[b]{2}{*}{.2038} & .50 & 1296 & 09310 & 8479 & \\
\hline & & .55 & 1424 & 09724 & 8185 & \\
\hline \multirow{2}{*}{0.6} & \multirow{2}{*}{$.26>9$} & .60 & 1558 & .1007 & 7862 & \\
\hline & & .65 & 1700 & .1036 & 7510 & \\
\hline \multirow{2}{*}{0.7} & \multirow{2}{*}{.3307} & .70 & 1852 & .1059 & 7132 & \\
\hline & & .75 & 2018 & .1077 & .6732 & \\
\hline \multirow{2}{*}{0.8} & \multirow{2}{*}{.3889} & .80 & 2200 & 1091 & .6314 & \\
\hline & & .85 & .2402 & 1102 & 5883 & \\
\hline \multirow{2}{*}{0.9} & \multirow{2}{*}{.4377} & .90 & 2626 & 1109 & $.5 \times 86$ & \\
\hline & & .95 & .2876 & $.1 / 13$ & .5007 & \\
\hline \multirow{2}{*}{1.0} & \multirow{2}{*}{.4680} & 1.0 & .3155 & .1115 & .4574 & \\
\hline & & & & & & \\
\hline
\end{tabular}


1. Sacks, A. H., Burnel1, J. A., "Ducted Propellers A Critical Review of the State of the Art," Progress in Aeronautical Sciences, Vol. III, pp. 87-135, Pergamon Press, Inc., New York, N. Y., 1962.

2. Tachmintij, A. J., "The Potential Problem of the Optimum Propeller with Finite Number of Blades Operating in a Cylindrical Duct," Report 1228, July 1958, David Taylor Model Basin, Washington, D. C.

3. Theodorsen, T., "Theoretical Investigation of Ducted Propeller Aerodynamics," Vol. III TREC TR 61-119, Sept. 1961, U. S. Army Transportation Research Command, Fort Eustis, Va.

4. Gray, R. B., "An Investigation of an Approach to the Problem of Determining the Optimum Design of Shrouded Propellers," TREC 60-44, May 1960, U. S. Army Transportation Research Command, Fort Eustis, Va.

5. Gray, R. B., "An Investigation of a Digital Computer Method of Determining the Optimum Design Parameters of Shrouded Propellers," TREC 61-124, Oct. 1961, U. S. Army Transportation Research Command, Fort Eustis, Va.

6. Morgan, W. B., "Theory of the Annular Airfoil and Ducted Propeller," Fourth Symposium on Naval Hydrodynamics, Office of Naval Research, Department of the Navy, Washington, D.C., ACR-73, Vol. I, pp. 161-212.

7. Ordway, D. C., Sluyter, M. M., and Sonnerup, B. O. U., "Three-Dimensional Theory of Ducted Propellers," TAR-TR 602, Aug. 1960, Therm Advancen Research Division of Therm Corporation, Ithaca, New York.

8. Chaplin, H. R., "A Method for Numerical Calculation of Slipstream Contraction of a Shrouded Impulse Disc in the Static Case with Application to other Axisymmetric Flow Problems," Report 1857, June 1964, David Taylor Model Basin, Washington, D. C.

9. Betz, A., "Screw Propellers with Minimum Energy Loss," Techrical Translation 736, National Research Council of Canada, Ottawa, Canada, 1958. 
10. Goldstein, S., "On the Vortex Theory of Screw Propellers," Proceedings of the Royal Society (London), Ser. A., Vol. 112 , pp. $440-465$.

11. Theodorsen, T., Theory of Propellers, McGraw-Hill Book Company, Inc., New York, N. Y. 1948.

12. Lamb, H., Hydrodynamics, Dover Publications, New York, N. Y., 1945 .

13. Gray, K. E., "Analysis of a Heavily Load Ducte Fan with an Infinite Number of Blades," to be publishei.

14. Fadecva, V. N., Computational Methods of Linear Algebra, pp. 67-72, Dover Publications, Inc., New York, N. Y. 1959.

15. Hodgman, C. D., Standard Mathematical Tables, The Chemical Rubber Publishing Company, Cleveland, Ohio, 1959.

16. Keuthe, A. M., Schetzer, J. C., Foundations of Aerodynamics, John Wiley and Sons, Inc., New York, N. Y., 1959. 
VITA

Terry Wriknt was born on February y, 1938, in Pinckneyville, Illinois. He attende public schools in Arkansas, Louisiana, Mississippi, Alabama and Virsinia.

He enterel the Georgia Institute of Technology in the winter of 1960 and was graduated with honors from the school of Aerospace Engineering in June of 1964. He continued his education as a graduate student at the Georeia Institute of Technology in September, 1964 and was awaraw the ingree of Master of Science in Aerospace En jineering in thun, 1.56.

He is a member of Sigma Gamma Tau, Tau Beta Pi, Phi Kappa Thi and Sigma Xi, and had a NASA Trainceship from September, 1y64, to Siptember, 1967.

He is married to the former Mary Anne Jackson. 